Universidade de São Paulo

Instituto de Física

\title{
Transição Quântico-Clássica em Sistemas com Propensão Coletiva
}

\author{
Milton Alexandre da Silva Junior
}

Orientador: Prof. Dr. Antonio Fernando Ribeiro de Toledo Piza

Tese de doutorado apresentada ao Instituto

de Física para a obtenção do título de

Doutor em Ciências

\section{Comissão Examinadora:}

Prof. Dr. Antonio Fernando Ribeiro de Toledo Piza (IFUSP)

Prof. Dr. Mahir Saleh Hussein (IFUSP)

Prof. Dr. Iberê Luiz Caldas (IFUSP)

Prof. Dr. Diógenes Galetti (IFT-Unesp)

Profa. Dra. Kyoko Furuya (IFGW-Unicamp)

São Paulo 


\section{Agradecimentos}

- Agradeço ao professor Antonio Fernando Ribeiro de Toledo Piza pela excelente orientação e pelo apoio, em especial pela paciência, a mim dispensados durante a realização deste trabalho.

- Agradeço à Fapesp pelo suporte financeiro durante a realização deste trabalho.

- Agradeço aos meus pais, Milton Alexandre da Silva e Maria de Lourdes Barcellos da Silva pelo apoio durante a realização deste trabalho.

- Agradeço aos colegas do Instituto de Física pelo apoio e pelas discussões, sobre Física ou não, que muito contribuíram para o desenvolvimento deste trabalho.

- Agradeço ao professor Carlos Molina Mendes pelo suporte computacional durante a realização deste trabalho. 


\section{Resumo}

Nesta tese, estudamos o comportamento de sistema quânticos cujo limite clássico pode tanto apresentar um comportamento regular como caótico. Nosso estudo se baseou na caracterização dinâmica do comportamento, caótico ou regular, destes sistemas quânticos no limite clássico através da análise da estrutura de distribuições no espaço de fases. Particularmente, aplicamos estas ferramentas ao estudo da transição quântico-clássica para uma classe de sistemas, que denominamos genericamente como "sistemas com propensão coletiva", os quais possuem a importante propriedade de admitir, em um certo limite análogo ao "limite termodinâmico", uma descrição de sua dinâmica em termos de um número reduzido de variáveis de natureza coletiva. Esta propriedade além de nos permitir "reduzir" a dinâmica de sistemas complicados à dinâmica de sistemas com poucos graus de liberdade, também nos permite, através de um escalonamento apropriado das variáveis coletivas, estudar o limite clássico destes sistemas concomitantemente com o "limite termodinâmico", evitando assim lidar diretamente com procedimentos formais usualmente associados ao limite clássico da forma " $\hbar \rightarrow 0$ ". Diferentemente das abordagens usuais, a abordagem que utilizamos nesta tese, baseada na propensão coletiva dos sistemas, permite a implementação de uma descrição em termos do comportamento de distribuições no espaço de fases para sistemas com espectro puramente discreto num espaço de dimensão finita. 


\begin{abstract}
In this thesis, we studied the behaviour of quantum systems which in the classical limit may exhibit either regular or chaotic behaviour. Our study was based on the dynamical characterization of the, chaotic or regular, behaviour of these systems in the classical limit through the analysis of the structure of phase-space distributions functions. Particularly, we have applied this characterization tools to the study of the quantum-to-classical transition in a class of quantum systems, that we called generally as "systems with collective propensity", which have the important property of admiting, in a certain limit analogous to the "thermodynamical limit", a description of their dynamics in terms of a low number of variables of collective nature. This property allows us to describe the dynamics of very complicated systems in terms of the dynamics of systems with few degrees of freedom. Besides, this allows us to study the classical limit of these systems concomitantly with the "termodynamical limit", avoiding in this way to deal with formal procedures associated with the limit " $\hbar \rightarrow 0$ ". Differently from the usual approaches, the one we employ in this thesis, based on the systems' collective propensity, allows for an implementation of a description in terms of the behaviour of phase-space distribution functions for systems endowed with a discrete spectrum in a finite-dimensional space.
\end{abstract}




\section{Sumário}

1 Introdução $\quad 3$

2 Expoentes de Lyapunov, Caos e a Dinâmica de Distribuições no Espaço de Fases 7

2.1 Expoentes de Lyapunov para um Tempo Finito em Termos do Gradiente de Distribuições no Espaço de Fases . . . . . . . . . . . . . . . . . . . . . . . . . . . 7

2.1.1 Distribuição Clássicas. . . . . . . . . . . . . . . . . . 7

2.1.2 Análogo Quântico do Expoente de Lyapunov para um Tempo Finito. . . . . 12

2.2 Heterogeneidade Distribucional no Espaço de Fases . . . . . . . . . . . . . . . . . 15

2.2.1 Contexto Clássico . . . . . . . . . . . . . . . . . . . 15

2.2.2 Contexto Quântico ....................... 17

3 Espaços de Dimensão Finita $r$

3.1 Base de Operadores Unitários de Schwinger . . . . . . . . . . . . . . . . . . . . 21

3.2 Formalismo no Espaço de Fases Discreto . . . . . . . . . . . . . . . . . . . . . 25

3.3 Distribuições num Espaço de Fases Discreto . . . . . . . . . . . . . . . . . . . . . . 28

4 Modelo com Propensão Coletiva $\quad 33$

4.1 Modelo de Bose-Hubbard com Dois Sítios e N Bósons . . . . . . . . . . . . . . . . . 33

4.1.1 Representação em Termos de Operadores Coletivos (Quasi-Spin) . . . . . . . 34

4.1.2 Transformada de Weyl Discreta e a Função de Wigner . . . . . . . . . . . . 36

4.1 .3 Limite Semi-Clássico . . . . . . . . . . . . . . . . . . . . . . . . 44

4.2 Heterogeneidade Distribucional no Modelo de Bose-Hubbard . . . . . . . . . . . . . 47

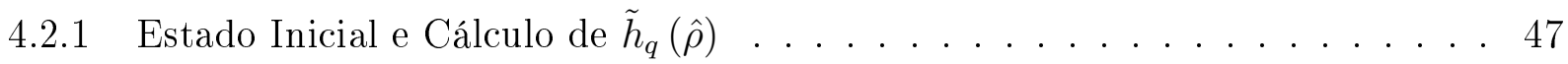

4.2.2 Correspondência Quântico-Clássica . . . . . . . . . . . . 54

5 Modelo com Dois Graus de Liberdade $\quad 60$

5.1 Hamiltoniano do Sistema, Representação de Quasi-Spin e Limite Termodinâmico . . 60

5.2 Sistema de Dois Momentos Angulares Acoplados . . . . . . . . . . . . . . . . . . 66

5.2.1 Hamiltoniano do Sistema e seu Limite Clássico . . . . . . . . . . . . . . . . 66 
5.2.2 Equivalência Entre o Modelo de Dois Momentos Angulares Acoplados e o Modelo (5.1) . . . . . . . . . . . . . . . . . . 67

5.3 Heterogeneidade Distribucional para o Modelo de Dois Momentos Angulares Aco-

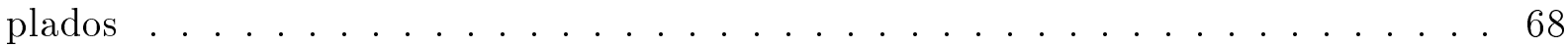

6 Conclusões e Perspectivas $\quad 75$

$\begin{array}{ll}\text { Apêndices } & 78\end{array}$

A. Exemplo Explícito da Equivalência entre $\tilde{h}_{q}(\hat{\rho})$ e $h_{q}(\hat{\rho})$ no Limite $D \rightarrow \infty$ e da Não Comutatividade dos Limites $D \rightarrow \infty$ e $\alpha \rightarrow 0$. . . . . . . . . . . . . . 78

B. Análise da variancia $v(\alpha)$ no Caso Contínuo e Obtenção da Equação $(2.41)(2)$. . . . . 82

C. Obtenção de $(2.45)(3)$ a partir de (3.39) . . . . . . . . . . . . . . . . . . . . 84

$\begin{array}{lr}\text { Referências Bibliográficas } & 87\end{array}$ 


\section{Capítulo 1}

\section{Introdução}

As profundas diferenças tanto conceituais quanto técnicas entre a mecânica quântica e a mecânica clássica, a existência de notáveis propriedades de "correspondência" entre as duas teorias e o sucesso de ambas nos seus respectivos domínios naturais de aplicação [1] constituem o cerne do conjunto de problemas designado genericamente como "transição quântico-clássica". Uma das questões cruciais nesse contexto é a que se refere à transição quântico-clássica para sistemas "classicamente não integráveis", sendo a não integrabilidade clássica no mínimo embaraçosa frente à linearidade quântica. Esta questão, por sua vez, deve ser entendida como indissoluvelmente ligada à questão da natureza específica do processo limite que conduz do sistema quântico ao sistema clássico não integrável.

Neste contexto, existem sistemas quânticos que, embora ideais, podem ser tomados como aproximações úteis para sistemas experimentalmente realizáveis, descritos por hamiltonianos que adquirem características clássicas, com um número restrito de variáveis dinâmicas de natureza coletiva, em um determinado limite que pode ser visto de certa forma como análogo a um "limite termodinâmico" [2, 3]. Tais sistemas serão chamados aqui "sistemas com propensão coletiva". Uma característica interessante dessa classe de sistemas, especialmente em conexão com análises da transição quântico-clássica, é a de que o "limite clássico" está associado a um limite do tipo "limite termodinâmico" bem definido, independentemente de operações formais do tipo " $\hbar \rightarrow 0$ ".

Um exemplo desse tipo de sistema consiste em uma aproximação de dois modos para um sistema frio de muitos $(N)$ bósons aprisionados em um poço duplo de potencial externo. Neste caso, se $a_{ \pm}^{\dagger}$ e $a_{ \pm}$são operadores de criação e aniquilação para os dois estados do dubleto mais baixo de partícula única, o hamiltoniano $H$ do sistema pode ser escrito em termos dos operadores

coletivos $J_{1} \pm \mathrm{i} J_{2}=a_{ \pm}^{\dagger} a_{\mp}, J_{3}=\left(a_{+}^{\dagger} a_{+}-a_{-}^{\dagger} a_{-}\right) / 2$ e $J=\left(a_{+}^{\dagger} a_{+}+a_{-}^{\dagger} a_{-}\right) / 2=\hat{N} / 2$; isto faz com que dinâmica do sistema de $N$ bósons seja descrita através da dinâmica de um sistema com apenas um grau de liberdade, descrito por um conjunto pequeno de variáveis dinâmicas coletivas. Note que neste caso, o número de variáveis dinâmicas as quais a dinâmica do sistema de muitos corpos fica reduzida, não depende do valor de $N$. 
No limite em que a constante de movimento $J \rightarrow \infty$, temos que ocorre um adensamento no espectro dos operadores escalonados $j_{k} \equiv J_{k} / J, k=1,2,3$; e de acordo com as relações de comutação satisfeitas pelos operadores $J_{k},\left[J_{i}, J_{j}\right]=\mathrm{i} \varepsilon_{i j k} J_{k}$, que em termos dos $j_{k}$ ficam $J\left[j_{i}, j_{j}\right]=$ $\mathrm{i} \varepsilon_{i j k} j_{k}$, vemos os operadores $j_{k}$ comutam no limite $J \rightarrow \infty$, o que possibilita que o espectro dos operadores $j_{k}$ sejam tratados como variáveis clássicas contínuas. Neste sentido, o hamiltoniano por partícula $H / 2 J$ pode então ser escrito em termos dos operadores escalonados $j_{k}$ de tal forma que, no limite $J \rightarrow \infty$, este adquire características clássicas. Como veremos no corpo desta tese, o escalonamento do hamiltoniano $H$ com o número de partículas dos sistema $N$, desempenha um papel fundamental para que se caracterize a propensão coletiva do sistema, ou seja, para que no "limite termodinâmico" $N \rightarrow \infty$ o sistema adquira características clássicas. Veremos também que caso tomássemos o limite $N \rightarrow \infty$ sem considerar o escalonamento de $H$, por exemplo da forma como foi feito em $[4,5,6]$, obteríamos uma descrição de $H$ em termos de variáveis contínuas num espaço de dimensão infinita ao invés de um sistema com características clássicas.

O sistema descrito no exemplo acima foi efetivamente realizado em uma versão "aberta" (incluindo dependências temporais externas notadamente do parâmetro de "hopping") e teve seu comportamento caótico estudado experimentalmente [7, 8, 9]. A generalização para redes com muitos sítios do modelo de dois modos, atualmente de uso corrente para a descrição de condensados atômicos de Bose-Einstein submetidos a redes periódicas de potencial externo geradas opticamente, consiste no modelo de Bose-Hubbard. Neste caso, a restrição aos dois modos mais baixos é substituída pela restrição aos $L$ modos mais baixos no caso de sistemas com $L$ sítios, e pela restrição à primeira zona de Brillouin para redes periódicas extensas. O caso $L>2$, finito, foi recentemente estudado do ponto de vista do "caos quântico" [10] caracterizado em termos de estatísticas de espaçamento de energias de estados estacionários.

Diversos trabalhos ao longo dos últimos quinze anos (ver por exemplo [11]-[20]) se ocuparam da transição quântico-clássica a partir de uma variedade de pontos de vista. Um desses pontos de vista tende a privilegiar o comportamento de trajetórias clássicas e relacioná-lo seja com proprideades de estados quânticos de sistemas "correspondentes", seja com as "trajetórias" descritas por valores médios quânticos das variáveis dinâmicas apropriadas [11, 20]. Alternativamente, a ênfase da descrição é deslocada das trajetórias para o comportamento de distribuições no espaço de fases, caso em que as dinâmicas clássica e quântica são descritas pelas equações de Liouville e de Liouvillevon Neumann respectivamente [12]-[19]. Em particular, o sistema considerado nos trabalhos de Emerson e Ballentine [13, 14] é um sistema aberto, com propensão coletiva redutível a um único grau de liberdade. Ele contém uma "simetria modular" que não é tratada na versão clássica, embora possa ser tomada em conta de forma a levar a diferentes formas de correspondência quântico-clássica [21].

Os trabalhos [15]-[19], por outro lado, enfatizam o comportamento de texturas no espaço de fases tanto no caso quântico (através do uso da representação de Wigner) como no caso clássico. Esse ponto de vista permite, particularmente em sua realização tal como desenvolvida nos trabalhos de 
Gu e Brumer [16]-[19], a construção de "medidas de complexidade" da textura no caso clássico que permite uma tradução essencialmente direta e independente de representação a sistemas quânticos, descritos em termos do operador densidade.

Em sua versão clássica [18], uma dessas medidas [16, 18, 19] se relaciona diretamente com o expoente de Lyapunov máximo do sistema, e este fato é usado na construção de um objeto dependente do tempo que pode ser visto como uma versão para um tempo finito deste expoente de Lyapunov. Uma característica relevante dessa medida de complexidade da distribuição no espaço de fases é o seu caráter não linear no operador densidade, o que em particular a torna sensível a processos de emaranhamento envolvendo diferentes graus de liberdade dos sistema considerado [22].

Estas ferramentas de análise do comportamento de distribuições no espaço de fases foram amplamente utilizadas no contexto de sistemas quânticos descritos em espaços de Hilbert com dimensão infinita,para versões quantizadas de sistemas clássicos conhecidos. Neste caso, a dinâmica do sistema quântico é descrita em termos de variáveis canônicas quânticas que possuem um espectro contínuo, tipicamente, variáveis de posição $q$ e seu momento canônicamente conjugado $p$ $[16,18,23,24,19,25,26]$. No entanto, outros casos, por exemplo envolvendo variáveis de ângulo e momento angular (ação), permanecem consideravelmente menos explorados.

De particular interesse para nós nesta tese, são os sistemas descritos num espaço Hilbert que possui dimensão $D$ finita, cujo limite clássico está diretamente associado ao "limite contínuo" $D \rightarrow \infty$, tomado de forma apropriada (por exemplo, o modelo de muitos bósons em dois modos localizados descrito anteriormente). Seria portanto desejável encontrar uma extensão da descrição em termos de distribuições no espaço de fases que também levasse em conta o caso de sistemas descritos em espaços de dimensão finita. Desta forma, seríamos capazes de aplicar as ferramentas de distribuições no espaço de fases também para esta classe sistemas, por exemplo sistemas descritos por graus de liberdade de spin. Na verdade, como veremos no corpo desta tese, é possível extender o uso desta análise de texturas de distribuições para o caso mais geral de sistemas descritos por graus de liberdade que não possuem uma "contrapartida" clássica, ou seja, que são descritios por um número finito de estados. Esta possibilidade se mostra potencialmente interessante quando nos voltamos para o contexto de sistema quânticos de muitos corpos, por exemplo um sistema de muitos átomos armadilhados como é o caso do modelo de Bose-Hubbard.

A idéia envolvida nesta tese consiste em utilizar a caracterização dinâmica do comportamento caótico baseada na análise da estrutura de distribuições no espaço de fases [16]-[19] para estudar, nesses sistemas com propensão coletiva, a transição quântico-clássica concomitante com o "limite termodinâmico". A transição está ligada, no caso, à crescente "macroscopicidade" do sistema, independendo de procedimentos formais do tipo associado ao limite " $\hbar \rightarrow 0$ ". Deve ser notada a grande liberdade em geral existente, neste contexto, com relação à escolha das variáveis dinâmicas coletivas a que se reduzirá a dinâmica do sistema, bem como a possibilidade de implementar descrições em termos de distribuições no espaço de fases mesmo no caso de um espectro puramente 
discreto num espaço de dimensão finita [2, 21].

Deve finalmente ser notado que a liberdade existente na escolha das particulares variáveis dinâmicas coletivas a serem privilegiadas no processo de transição quântico-clássica [21] implica na possibilidade de alterar concomitantemente a representação no espaço de fases dos estados estacionários quânticos, e portanto também o conteúdo espectral, e consequentemente as características dinâmicas associadas a diferentes regiões do espaço de fases. Desta forma, é no mínimo extremamente duvidoso que seja possível formar uma imagem razoavelmente completa da transição quântico-clássica sem levar em conta a liberdade inerente à escolha das variáveis dinâmicas finais.

No que segue dividimos esta tese da seguinte maneira: no capítulo 2, apresentamos um breve resumo das ferramentas utilizadas na análise de distribuições no espaço de fases. No capítulo 3, introduzimos o formalismo necessário para "traduzir" estas ferramentas do espaço de fases para o contexto de sistemas descritos num espaço de dimensão finita. Finalmente no capítulo 4, aplicamos estas ferramentas para o caso de um sistema com propensão coletiva (o modelo de Bose-Hubbard com dois sítios e $N$ bósons) cuja comportamento no limite clássico é totalmente regular. No capítulo 5, realizamos mesmo estudo do capítulo 4 para um sistema com propensão coletiva que, no limite clássico, pode apresentar um comportamento irregular (caótico). No capítulo 6, apresentamos algumas conlcusões e discutimos algumas perspectivas de trabalhos futuros dentro do assunto desta tese.

No apêndice A, apresentamos um exemplo explícito de como é possível obter a heterogeneidade distribucional no caso de um espaço de dimensão infinita tomando como ponto de partida o equivalente desta para um espaço de dimensão finita, através de um "limite contínuo". Neste apêndice também apresentamos um exemplo explícito da não comutatividade, no caso de um espaço de dimensão finita, entre o limite clássico e o limite que define a heterogeneidade distribucional. No apêndice B, fazemos uma análise do comportamento da variancia utilizada na definição da heterogeneidade distribucional dependência da heterogeneidade no espaço de fases no caso de um espaço de dimensão finita. No apêndice C, obtemos de uma forma mais explícita a expressão para heterogeneidade distribucional no caso de um espaço de dimensão finita. 


\section{Capítulo 2}

\section{Expoentes de Lyapunov, Caos e a Dinâmica de Distribuições no Espaço de Fases}

Neste capítulo, apresentaremos de uma forma resumida a teoria descrita em[16, 18], na qual nos baseamos para fazer o estudo da transição quântico-clássica. Apresentaremos a definição de uma medida de complexidade no espaço de fases, que têm como principal ingrediente o gradiente de distribuições neste espaço [18]. Em seguida apresentaremos uma definição alternativa desta medida [16] que, apesar de também recair na utilização de gradiente de distribuições, tem como principal ingrediente uma medida de entropia. Esta segunda abordagem será a que utilizaremos em nosso estudo da transição quântico-clássica, em primeiro lugar por apresentar uma essência mais física ao se basear numa medida de entropia e em segundo lugar, pelo mesmo motivo, por permitir uma conexão mais imediata com o estudo de processos decoerência [22].

\subsection{Expoentes de Lyapunov para um Tempo Finito em Ter- mos do Gradiente de Distribuições no Espaço de Fases}

\subsubsection{Distribuição Clássicas.}

O caos em mecânica clássica é usualmente definido através da forte sensibilidade das trajetórias no espaço de fases a pequenas mudanças nas suas condições iniciais. Quantitativamente, o caos é descrito por uma grandeza positiva conhecida como expoente de Lyapunov máximo. Esta grandeza mede a taxa com que duas trajetórias no espaço de fases, oriundas de condições iniciais infinitesimalmente próximas, se afastam após um tempo muito longo $(t \rightarrow \infty)$.

$\mathrm{Na}$ verdade existe todo um espectro de expoentes de Lyapunov (o número de expoentes é igual ao número de dimensões do espaço de fases), pois as taxas com que as trajetórias se afastam uma da outra podem variar de acordo com a direção da separação inicial no espaço de fases. No entanto é comum nos referirmos apenas ao maior deles, pois um vetor de separação inicial genérico 
necessariamente contém uma componente na direção associada ao expoente de Lyapunov máximo e devido à taxa de crescimento exponencial o efeito devido aos demais expoentes será relativamente suprimido conforme $t \rightarrow \infty$.

Tendo isso em mente, consideremos inicialmente um sistema Hamiltoniano com $n$ graus de liberdade representados pelas variáveis canônicas de posição $\tilde{q}_{1}, \ldots, \tilde{q}_{n}$ e momento $\tilde{p}_{1}, \ldots, \tilde{p}_{n}$, onde $\tilde{q}_{i}$ e $\tilde{p}_{i}$ são canonicamente conjugadas, ou seja, $\left\{\tilde{q}_{i}, \tilde{p}_{i}\right\}_{\left(\tilde{q}_{i}, \tilde{p}_{i}\right)}=1$, onde $\{f, g\}_{\left(\tilde{q}_{i}, \tilde{p}_{i}\right)}=\sum_{i=1}^{n}\left[\left(\partial f / \partial \tilde{q}_{i}\right)\left(\partial g / \partial \tilde{p}_{i}\right)\right.$ - $\left.\left(\partial f / \partial \tilde{p}_{i}\right)\left(\partial g / \partial \tilde{q}_{i}\right)\right]$, são os parênteses de Poisson nas variáveis canônicas $\tilde{q}_{i}$ e $\tilde{p}_{i}$. Definimos então variáveis escalonadas adimensionais $q_{1}, \ldots, q_{n}$ e $p_{1}, \ldots, p_{n}$, tais que $q_{i} \equiv \tilde{q}_{i} / \ell$ e $p_{i} \equiv \tilde{p}_{i} \ell / \varkappa$, onde $\ell$ possui unidades de posição $\tilde{q}_{i}$ e $\varkappa$ possui unidades de ação, ou seja, de $\tilde{q}_{i} \times \tilde{p}_{i}$. Neste caso, podemos facilmente verificar que $\left\{q_{i}, p_{i}\right\}_{\left(\tilde{q}_{i}, \tilde{p}_{i}\right)}=1 / \varkappa$ e $\left\{q_{i}, p_{i}\right\}_{\left(q_{i}, p_{i}\right)}=1 .^{1}$

Afim de tratarmos igualmente as coordenadas $q_{i}$ e seus respectivos momentos conjugados $p_{i}$, caracterizamos um ponto no espaço de fases por um vetor no espaço $2 n$-dimensional

$$
\gamma \equiv\left(q_{1}, \ldots, q_{n}, p_{1}, \ldots, p_{n}\right)
$$

Em seguida, por conveniência, introduzimos a matriz antisimétrica $\mathbf{J}$

$$
\mathbf{J}=\left(\begin{array}{cc}
\mathbf{0} & \mathbf{1} \\
-\mathbf{1} & \mathbf{0}
\end{array}\right)
$$

onde $\mathbf{0}$ e $\mathbf{1}$ são as matrizes nula e identidade $n \times n$, respectivamente. A matriz $\mathbf{J}$ obedece à relação

$$
\mathbf{J}^{\dagger}=\mathbf{J}^{-1}=-\mathbf{J}
$$

Com o auxílio de $\mathbf{J}$, as equações canônicas de movimento (ou seja, as equações de Hamilton), neste caso podem ser escritas na forma compacta

$$
\dot{\gamma}=\mathbf{J}(\nabla H) .
$$

A sensibilidade de uma trajetória clássica $\gamma(t)$ a pequenas perturbações em sua condição iniciail $\gamma(0)$ é descrita pela matriz de estabilidade $\mathbf{M}_{\gamma(0)}$, cujos elementos são definidos como

$$
\mathrm{M}_{i j}[\gamma(0), t] \equiv \frac{\partial \gamma_{i}(t)}{\partial \gamma_{j}(0)},
$$

ou seja, se considerarmos duas trajetórias $\gamma_{1}(t)$ e $\gamma_{2}(t)$ infinitesimalmente separadas inicialmente, teremos que a evolução desta separação será dada por

$$
\gamma_{2}(t)-\gamma_{1}(t)=\mathbf{M}_{\gamma_{1}(0)}\left\{\gamma_{2}(0)-\gamma_{1}(0)\right\}
$$

\footnotetext{
${ }^{1}$ Para descrições quânticas, fazendo a associação direta $\left(q_{i}, p_{i}\right) \rightarrow\left(\hat{q}_{i}, \hat{p}_{i}\right)$ entre as variáveis adimensionais $q_{i}$ e $p_{i}$ e os operadores $\hat{q}_{i}$ e $\hat{p}_{i}$, temos que $\left[\hat{q}_{i}, \hat{p}_{i}\right]=\mathrm{i} \hbar / \varkappa, \hbar / \varkappa$ pode ser visto como uma espécie de constante de Planck adimensional.
} 
A matriz de estabilidade $\mathbf{M}_{\gamma(0)}$ também pode ser computada através de sua equação diferencial. Esta equação diferencial segue diretamente de (2.4) e (2.5)

$$
\dot{\mathbf{M}}_{\gamma(0)}=\mathbf{J}\left(\nabla^{2} H\right) \mathbf{M}_{\gamma(0)},
$$

com a condição $\mathbf{M}_{\gamma(0)}=\mathbf{1}$, em $t=0$.

Com isso podemos enfim definir o expoente de Lyapunov máximo do sistema. Uma forma de definir os expoentes de Lyapunov é através dos autovalores da matriz [27]

$$
\Lambda_{\gamma(0)}=\lim _{t \rightarrow \infty}\left(\mathbf{M}_{\gamma(0)}^{T} \mathbf{M}_{\gamma(0)}\right)^{1 / 2 t}
$$

onde $\mathbf{M}_{\gamma(0)}^{T}$ representa a transposta de $\mathbf{M}_{\gamma(0)}$. Neste caso, os expoentes de Lyapunov são os logaritmos dos autovalores de $\Lambda_{\gamma(0)}$ e segundo [28, 27, 29] é possível provar que o limite

$$
\lim _{t \rightarrow \infty} \frac{1}{t} \ln \left\{\left|\mathbf{M}_{\gamma(0)} u\right|\right\}=\lambda[\gamma(0)]
$$

onde $|\cdots|$ é a norma usual no espaço de fases, existe para qualquer vetor $u$ do espaço de fases, tal que $u \neq 0$. Na equação (2.9), $\lambda[\gamma(0)]$ é o maior dos expoentes de Lyapunov do sistema, ou seja, o expoente de Lyapunov máximo.

Existe uma forma mais geral de definir os expoentes de Lyapunov. Seja $\mathcal{M}$ uma variedade Riemanniana $2 n$-dimensional, compacta e diferenciável. Denotamos por $\mathcal{T}_{x}$ o espaço tangente a $\mathcal{M}$ num dado ponto $x$. O teorema de Oseledec $[28,27]$ nos diz que para todo vetor $\eta \in \mathcal{T}_{x}, \eta \neq 0$, o limite

$$
\lim _{t \rightarrow \infty} \frac{1}{t} \ln \left\{\left\|T_{x}^{t} \eta\right\|\right\}=\lambda(x, \eta),
$$

existe e é finito, onde $\|\cdots\|$ é a norma induzida em $\mathcal{T}_{x}$ pela métrica Riemanniana em $\mathcal{M}$ e $T_{x}^{t}$ é responsável pelo mapeamento de vetores de $\mathcal{T}_{x}$ em vetores de $\mathcal{T}_{x(t)}$, onde $\{x(t)\}$ é uma curva em $\mathcal{M}$ tal que $x(0)=x$.

Além disso, o teorema também nos diz que existe uma base $\left\{\eta_{i}\right\}_{1 \leq i \leq 2 n}$ de $\mathcal{T}_{x}$ tal que, conforme $\eta$ varia o número $\lambda(x, \eta)$ assume apenas os valores $\left\{\lambda\left(x, \eta_{i}\right)\right\}_{1 \leq i \leq 2 n}$, com

$$
\lambda_{1}(x) \leq \lambda_{2}(x) \leq \cdots \leq \lambda_{2 n}(x)
$$

onde fizemos $\lambda\left(x, \eta_{i}\right)=\lambda_{i}(x), 1 \leq i \leq 2 n$.

Os números $\left\{\lambda_{i}(x)\right\}_{1 \leq i \leq 2 n}$ formam o chamado espectro de expoentes de Lyapunov da evolução do sistema no ponto $x$ e $\lambda_{2 n}(x)$ é o expoente de Lyapunov máximo do sistema. Segundo [28] (teorema $\mathrm{B}$ ), para um dado vetor $\eta \neq 0$ tomado aleatoriamente no espaço tangente, teremos que $\lambda(x, \eta) \equiv \lambda_{2 n}(x)$, uma vez que os vetores $\eta$ para os quais $\lambda(x, \eta)<\lambda_{2 n}(x, \eta)$ formam um conjunto de medida de Lebesgue nula. 
Seja agora $\rho_{t}[\gamma(t)]$ uma distribuição clássica de probabilidades bem comportada e $\delta \gamma(t)$ uma pequena variação desta trajetória. O teorema de Liouville implica que

$$
\rho_{t}[\gamma(t)+\delta \gamma(t)]-\rho_{t}[\gamma(t)]=\rho_{0}[\gamma(0)+\delta \gamma(0)]-\rho_{0}[\gamma(0)]
$$

No limite em que $|\delta \gamma(0)| \rightarrow 0$ e utilizando o fato de que $\mathbf{J}^{2}=-\mathbf{1}$ e $\mathbf{J} \mathbf{M}_{\gamma(0)}^{\dagger} \mathbf{J}=-\mathbf{M}_{\gamma(0)}^{-1}$, obtemos a equação

$$
\mathbf{M}_{\gamma(0)} \mathbf{J} \nabla \rho_{0}[\gamma(0)]=\mathbf{J} \nabla \rho_{t}[\gamma(t)]
$$

Substituindo (2.11) em (2.9) obtemos

$$
\begin{aligned}
\lambda[\gamma(0)] & =\lim _{t \rightarrow \infty} \frac{1}{t} \ln \left\{\left|\mathbf{J} \nabla \rho_{t}[\gamma(t)]\right|\right\} \\
& =\lim _{t \rightarrow \infty} \frac{1}{t} \ln \left\{\left|\nabla \rho_{t}[\gamma(t)]\right|\right\}
\end{aligned}
$$

É importante notar que (2.12) define o expoente de Lyapunov máximo não mais em termos de trajetórias mas agora em termos das propriedades de distribuições no espaço de fases [16, 18]. Mais precisamente, quanto mais rápido a estrutura de $\rho_{t}$ aumentar, maior será o valor de $\lambda[\gamma(0)]$.

Seguindo [19], definimos a seguinte quantidade,

$$
\chi_{c}(t) \equiv\left[\frac{\int\left|\nabla \rho_{t}\right|^{2} d \gamma}{\int \rho_{t}^{2} d \gamma}\right]^{1 / 2}
$$

que através de uma integração por partes se torna

$$
\chi_{c}(t)=\left[-\frac{\int \rho_{t}\left(\nabla^{2} \rho_{t}\right) d \gamma}{\int \rho_{t}^{2} d \gamma}\right]^{1 / 2}
$$

que por sua vez é uma média sobre os gradientes da distribuição $\rho_{t}$.

Utilizando (2.11) e (2.14), obtemos

$$
\chi_{c}(t)=\left[\frac{\int\left|\mathbf{J M}_{\gamma(0)} \mathbf{J}\left(\nabla \rho_{0}\right)\right|^{2} d \gamma}{\int \rho_{0}^{2} d \gamma}\right]^{1 / 2} .
$$

O significado físico de $\chi_{c}(t)$ se torna mais claro quando consideramos a transformada de Fourier de $\rho_{t}$, ou seja,

$$
\rho_{t}(\gamma)=\frac{1}{(2 \pi)^{4}} \int d \mathbf{k} \exp (2 \pi \mathrm{i} \mathbf{k} \cdot \gamma) \rho_{t}(\mathbf{k})
$$


onde $\rho_{t}(\mathbf{k})$ denota a componente de Fourier calculada no vetor $2 n$-dimensional $\mathbf{k}$. Neste caso temos que (2.14) fica

$$
\chi_{c}^{2}(t)=\frac{\int d \mathbf{k} \mathbf{k}^{2}\left|\rho_{t}(\mathbf{k})\right|^{2}}{\int d \mathbf{k}\left|\rho_{t}(\mathbf{k})\right|^{2}},
$$

mostrando que $\chi_{c}$ é o raio quadrático médio da transformada de Fourier da distribuição $\rho_{t}(\gamma)$, e que portanto pode ser utilizada como uma medida da estrutura no espaço de fases clássico [19]. Isto significa que quanto maior $\chi_{c}$, mais estrutura $\rho_{t}(\gamma)$ apresentará.

Para dinâmicas completamente integráveis existe um conjunto especial de coordenadas generalizadas formado por variáveis de ação e ângulo $I_{i}$ e $\theta_{i}$, com $i=1, \ldots, n$. Nesta representação, o Hamiltoniano depende somente daquelas variáveis de ação que são constantes de movimento. Para estes casos, a equação (2.7) possui a solução

$$
\mathbf{M}_{\gamma(0)}=\mathbf{1}+\mathbf{J} \frac{\partial^{2} H}{\partial \gamma^{2}} t .
$$

Substituindo esta dependência temporal explícita da matriz de estabilidade na equação (2.15) obtemos o comportamento de $\chi_{c}$ para tempos longos

$$
\lim _{t \rightarrow \infty} \frac{\chi_{c}(t)}{t}=\left[\frac{\int\left|\left(\partial^{2} H / \partial \gamma^{2}\right) \mathbf{J} \nabla \rho_{0}\right|^{2} d \gamma}{\int \rho_{0}^{2} d \gamma}\right]^{1 / 2} .
$$

Portanto, para dinâmicas regulares, a estrutura da distribuição $\rho_{t}$, medida por $\chi_{c}(t)$, assintoticamente apresenta uma dependência linear no tempo. Isto ocorre na representação de ação e ângulo, no entanto, $\chi_{c}(t)$ pode apresentar uma dependência polinomial no tempo para outras representações canônicas[19].

No caso em que o sistema em questão possui uma dinâmica caótica temos que [18]

$$
\lim _{t \rightarrow \infty} \frac{1}{t} \ln \chi_{c}(t)=\lambda
$$

onde $\lambda$ é o expoente de Lyapunov máximo do sistema. Isto significa que, no caso caótico, o raio quadrático médio da transformada de Fourier da distribuição $\rho_{t}$ aumenta assintoticamente a uma taxa exponencial dada por $\lambda$.

Uma vez que um dado limite de resolução $\delta$ no espaço de fases corresponde à impossibilidade de levarmos em consideração modos de Fourier maiores que $1 / \delta$, o caos pode ser interpretado como uma espécie de perda exponencial de informação, contida na expansão em modos de Fourier da distribuição inicial $\rho_{0}$.

Por definição, o expoente de Lyapunov generalizado $\lambda$ é uma propriedade assintótica, relevante quando $t \rightarrow \infty$. No entanto, de acordo com [18, 19], é possível definir uma versão do expoente de Lyapunov para um tempo $t$ finito em termos de $\chi_{c}(t)$. Esta definição é dada por 


$$
\lambda_{c}(t) \equiv \frac{1}{t} \ln \left[\frac{\chi_{c}(t)}{\chi_{c}(0)}\right]
$$

a qual deve satisfazer

$$
\lim _{t \rightarrow \infty} \lambda_{c}(t)=\lambda
$$

Esta definição ((2.20) com (2.21)) torna-se bastante relevante em um estudo realístico (numérico, para ser mais preciso) da correspondência quântico-clássica, pois num estudo deste tipo temos acesso apenas às propriedades da dinâmica clássica caótica que podem ser analisadas para um tempo $t$ finito. Portanto, tendo em mente este contexto realístico, é no mínimo extremamente útil ter em mãos uma definição do expoente de Lyapunov para um tempo $t$ finito.

A quantidade $\lambda_{c}(t)$, nos permite estudar o caos baseando-nos na dinâmica de distribuições no espaço de fases, apenas no caso de distribuições clássicas. Na subseção seguinte apresentaremos uma versão quântica de $\lambda_{c}(t)\left(\lambda_{q}(t)\right.$ dada em (2.28)) que nos permitirá extender a análise de distribuições no espaço de fases para o caso quântico.

\subsubsection{Análogo Quântico do Expoente de Lyapunov para um Tempo Fi- nito.}

Do ponto de vista de ensembles estatísticos, a maneira usual de estudarmos as propriedades de correspondência entre um sistema clássico e seu análogo quântico (sistema quantizado) consiste em comparamos a equação clássica de Liouville para uma densidade de probabilidades no espaço de fases, com a equação quântica de Liouville-von Neumann para o operador densidade, representando um dado estado do sistema quantizado, numa dada representação no espaço de fases, por exemplo a representação de Wigner-Weyl. Mais precisamente, suponhamos por exemplo um sistema hamiltoninano genérico da forma $H(p, q)=p^{2} / 2 m+V(q)$, descrito em termos das variáveis dinâmicas de posição $q$ e momento $p$. Classicamente, um estado inicial de $H(q, p)$ pode ser representado por uma distribuição de probabilidades positiva $\rho(p, q)$ no espaço espaço de fases enquanto que um estado inicial do sistema quantizado, descrito por um operador densidade $\hat{\rho}$, tem sua representação no espaço de fases dada pela função de Wigner correspondente $\rho^{W}(p, q)$, cuja definição é

$$
\rho^{W}(p, q) \equiv \frac{1}{2 \pi \hbar} \int d v \mathrm{e}^{\frac{\mathrm{i}}{\hbar} p v}\left\langle q-\frac{1}{2} v|\hat{\rho}| q+\frac{1}{2} v\right\rangle .
$$

A evolução temporal clássica e quântica deste sistema são então dadas respectivamente pelas equações de Liouville para $\rho(q, p)$ e de Liouville-von Neumann para $\rho^{W}(q, p)$, ou seja,

$$
\frac{\partial}{\partial t} \rho(p, q)=-\left[\frac{p}{m} \partial_{q}-\partial_{q} V(q) \partial_{p}\right] \rho(p, q)
$$




$$
\frac{\partial}{\partial t} \rho^{W}(p, q)=-\left[\frac{p}{m} \partial_{q}-\partial_{q} V(q) \partial_{p}\right] \rho^{W}(p, q)+\sum_{\lambda=1}^{\infty} \frac{(\hbar / 2 \mathrm{i})^{2 \lambda}}{(2 \lambda+1) !} \partial_{q}^{2 \lambda+1} V(q) \partial_{p}^{2 \lambda+1} \rho^{W}(p, q)
$$

desde que o potencial $V(q)$ possa ser expandido em série de Taylor. Classicamente, temos que o caso extremo $\rho(p, q)=\delta\left(p-p^{\prime}\right) \delta\left(q-q^{\prime}\right)$, quando substituido em (2.23), nos fornece as equações de Newton para o movimento de uma partícula de massa $m$. Quanticamente no entanto, esta forma "ultralocal" não é permitida pois , para o caso de um estado puro²,

$$
\int d p \int d q\left[\rho^{W}(p, q)\right]^{2}=\frac{1}{2 \pi \hbar}
$$

A impossibilidade de uma descrição da evolução do sistema quântico em termos de trajetórias de partículas no espaço de fases, está initimamente ligada ao princípio de incerteza de Heisenberg e é uma dificuldade que aparece naturalmente em estudos da correspondência quântico-clássica.

Contudo, apesar da dificuldade mencionada no parágrafo anterior, segundo [18, 19], é possível definir um "análogo quântico" para os expoentes de Lyapunov, da forma como foram definidos em (2.12), fazendo uso de funções de Wigner no espaço de fases, através de uma analogia direta com a equação (2.13). Para tanto, seguindo [18, 19], definimos a medida $\chi_{q}$ da estrutura de uma distribuição quântica no espaço de fases como

$$
\chi_{q} \equiv\left[\frac{\int\left|\nabla \rho_{t}^{W}(\gamma)\right|^{2} d \gamma}{\int\left(\rho_{t}^{W}(\gamma)\right)^{2} d \gamma}\right]^{1 / 2}
$$

onde $\rho^{W}(\gamma)$ é a função de Wigner dada em (2.22). No espaço de Fourier, temos que

$$
\chi_{q}^{2}=\frac{\int d \mathbf{k k}^{2}\left|\rho_{t}^{W}(\mathbf{k})\right|^{2}}{\int d \mathbf{k}\left|\rho_{t}^{W}(\mathbf{k})\right|^{2}}
$$

com

$$
\rho_{t}^{W}(\gamma)=\frac{1}{(2 \pi)^{4}} \int d \mathbf{k} \exp (2 \pi \mathrm{ik} \cdot \gamma) \rho_{t}^{W}(\mathbf{k})
$$

de onde vemos que $\chi_{q}$ é o raio quadrático médio da tranformada de Fourier da função de Wigner. Com a definição (2.26), temos finalmente que o expoente de Lyapunov para tempo finito em termos da dinâmica de distribuições quânticas, em analogia direta com $\lambda_{c}(t)$, é dado por

$$
\lambda_{q}(t) \equiv \frac{1}{t} \ln \left[\frac{\chi_{q}(t)}{\chi_{q}(0)}\right]
$$

Apesar de possuírem uma forma muito semelhante e serem obitidas através de um simples

\footnotetext{
${ }^{2}$ Para o caso de um estado de mistura, o lado direito da equação (2.25) ficaria multiplicado por $\sum_{k} w_{k}^{2}$, onde os $w_{k}$ são as probabilidades dos diferentes estados da mistura.
} 
analogia, as quantidades $\lambda_{c}(t)$ e $\lambda_{q}(t)$ possuem comportamentos diferentes no limite $t \rightarrow \infty$. Classicamente, vemos em (2.21) que neste limite $\lambda_{c}(t)$ por definição é o expoente de Lyapunov máximo do sistema. Quanticamente no entanto, a situação é um pouco diferente. Suponhamos que $\hat{\rho}$ seja o operador densidade associado à função de Wigner $\rho^{W}(q, p)$ e $\hat{\gamma}_{i}$ o operador associado à variável canônica clássica $\gamma_{i}$ (por exemplo $q_{i}$ ou $p_{i}$ ). Segundo [18], podemos considerar na equação (2.14) o fato de que $\nabla^{2} \rho \equiv\{p,\{p, \rho\}\}+\{q,\{q, \rho\}\}$ e além disso utilizar o processo usual de quantização $\{A, B\} \rightarrow-(\mathrm{i} / \hbar)[\hat{A}, \hat{B}]$ (onde [,] representa o comutador entre dois operadores) para obter uma versão de (2.26) em termos do operador densidade $\hat{\rho}$ e das variáveis canônicas do sistema $\hat{\gamma}_{i}$ dada por

$$
\chi_{q}^{2}=2 \sum_{i} \frac{\operatorname{Tr}\left(\hat{\rho}^{2} \hat{\gamma}_{i}^{2}-\hat{\rho} \hat{\gamma}_{i} \hat{\rho} \hat{\gamma}_{i}\right)}{\hbar^{2} \operatorname{Tr}\left(\hat{\rho}^{2}\right)}
$$

Esta versão é independente de representação e, para o caso de um estado puro, ou seja $\hat{\rho}^{2}=\hat{\rho}$, tem a forma mais simples

$$
\chi_{q}^{2}=\frac{2}{\hbar^{2}} \sum_{i}\left(\left\langle\hat{\gamma}_{i}^{2}\right\rangle-\left\langle\hat{\gamma}_{i}\right\rangle^{2}\right)
$$

onde $\langle\hat{O}\rangle \equiv\langle\psi|\hat{O}| \psi\rangle$ denota o valor esperado do operador $\hat{O}$ em um dado estado quântico $|\psi\rangle$. Uma vez que a equação (2.29) nos diz que

$$
\chi_{q}^{2} \leq 2 \sum_{i} \frac{\operatorname{Tr}\left(\hat{\rho}^{2} \hat{\gamma}_{i}^{2}\right)}{\hbar^{2} \operatorname{Tr}\left(\hat{\rho}^{2}\right)}
$$

temos que $\chi_{q}$ possui um limite superior para qualquer sistema Hamiltoniano limitado. Dessa forma, para sistemas limitados e $\hbar$ fixo, temos que

$$
\lim _{t \rightarrow+\infty} \lambda_{q}(t)=0
$$

que nada mais é do que o resultado conhecido de que sistemas quânticos limitados não podem exibir caos no sentido clássico.

Para estudar o comportamento de $\lambda_{q}(t)$ e $\lambda_{c}(t)$ para $t$ finito, é necessário estudar as soluções da equação (2.24) para função de Wigner $\rho^{W}$. Consideremos então, um sistema um sistema Hamiltoniano específico com dois graus de liberdade $H=H\left(q_{1}, q_{2}, p_{1}, p_{2}\right)$, sob a ação de um potencial $V\left(q_{1}, q_{2}\right)$. Neste caso, temos que $(2.24)$ é dada por [19]

$$
\frac{\partial \rho^{W}}{\partial t}=\left\{H, \rho^{W}\right\}+\sum_{\left(l_{1}+l_{2}\right)>1, \text { impar }} \frac{(\hbar / 2 \mathrm{i})^{\left(l_{1}+l_{2}-1\right)}}{l_{1} ! l_{2} !} \frac{\partial^{\left(l_{1}+l_{2}\right)} V\left(q_{1}, q_{2}\right)}{\partial q_{1}^{l_{1}} \partial q_{2}^{l_{2}}} \frac{\partial^{\left(l_{1}+l_{2}\right)} \rho^{W}}{\partial p_{1}^{l_{1}} \partial p_{2}^{l_{2}}},
$$

onde o primeiro termo do lado direito da equação são os parênteses de Poison clássicos e o segundo termo representa a soma sobre uma série infinita de "correções quânticas". 


\subsection{Heterogeneidade Distribucional no Espaço de Fases}

Os mesmos resultados da seção anterior podem ser obtidos a partir de uma abordagem ligeiramente diferente devida a $\mathrm{Gu}$ [16]. A dedução de Gu apresenta um caráter mais físico, pois se baseia numa medida da complexidade de uma distribuição ao longo do espaço de fases, e utiliza como medida para esta complexidade uma variação da entropia do sistema quando é feita uma média (coarsegraining) infinitesimal no espaço de fases. Utilizando esta variação de entropia, Gu [16] definiu uma medida capaz de descrever quantitativamente a variação na estrutura da distribuição no espaço de fases conforme é feita a média (coarse-graining), a heterogeneidade distribucional no espaço de fases. Como veremos mais adiante, esta quantidade está diretamente relacionada, em sua versão clássica $h_{c}$, com a quantidade $\chi_{c}$ dada em (2.13) e em sua versão quântica $h_{q}$ com $\chi_{q}$ dada em $(2.26)$.

\subsubsection{Contexto Clássico}

Com o objetivo de definir uma quantidade que pudesse ser utilizada como uma medida da "heterogeneidade microscópica" de uma densidade no espaço de fases, Yan Gu [16] tomou como ponto de partida uma analogia direta com o problema de agitar uma mistura inicialmente separada de água e tinta preta. Gu considerou que enquanto do ponto vista macrocópico o líquido inicialmente não uniforme tende a um estado de mistura com uma cor predominantemente cinza, do ponto de vista microscópico quantidades de água e tinta vão sendo deformadas em filamentos cada vez mais finos de tal forma que a escala da heterogeneidade, inicialmente macroscópica, se torne microscópica. Como em mecânica estatística quanto mais uniformemente distribuída é $\rho$ maior o valor da entropia de Gibbs $S$, poderíamos considerar a entropia média (coarse-grained) $S_{\text {cg }}$ como uma medida da "uniformidade macroscópica" de $\rho$.

Supondo agora um ensemble estatístico de sistemas clássicos que inicialmente estão uniformemente distribuídos em uma região pequena do espaço de fases. À medida que a densidade $\rho_{t}(\gamma)$, com $\gamma=\left(q_{1}, \ldots, q_{n}, p_{1}, \ldots, p_{n}\right)$, se espalha no espaço de fases em filamento cada vez mais finos, a diferença entre a entropia média (coarse-grained) e a entropia do sistema (fine-grained) apresentará um crescimento contínuo até que a escala da heterogeneidade distribucional de $\rho$ se torne comparável ao tamanho da célula do espaço de fases utilizada no processo de média (coarsegraining). Neste caso, podemos considerar esse aumento na entropia do sistema devido a uma média (coarse-graining) infinitesimal como uma medida da heterogeneidade distribucional de $\rho$.

Para uma dada densidade $\rho(\gamma)$ no espaço de fases, podemos definir uma média (coarse-graining) sobre esta densidade tal que forma que a densidade média $\rho_{\alpha}(\gamma)$ é dada por [16]

$$
\rho_{\alpha}(\gamma)=\int \delta_{\alpha}\left(\gamma^{\prime}\right) \rho\left(\gamma-\gamma^{\prime}\right) d \gamma^{\prime}
$$

onde $\lim _{\alpha \rightarrow 0} \rho_{\alpha}=\rho$ e $\delta_{\alpha}\left(\gamma^{\prime}\right)$ é uma função de "alisamento" não-negativa localizada em uma região 
de tamanho $\alpha$ em $\gamma^{\prime}$ e que possui as propriedades

$$
\begin{gathered}
\int \delta_{\alpha}(\gamma) d \gamma=1, \\
\delta_{\alpha}(-\gamma)=\delta_{\alpha}(\gamma), \\
\lim _{\alpha \rightarrow 0} \delta_{\alpha}(\gamma)=\delta(\gamma), \quad(\text { Delta de Dirac }) \\
\lim _{\alpha \rightarrow 0} \frac{1}{\alpha} \int \gamma_{i} \gamma_{j} \delta_{\alpha}(\gamma) d \gamma=\delta_{i j}, \quad(i, j=1, \ldots, 2 n)
\end{gathered}
$$

$\mathrm{e}$

$$
\int M(\gamma) \delta_{\alpha}(\gamma) d \gamma=o(\alpha)
$$

se $M(\gamma)$ for um monômio em $\gamma$ de ordem maior que dois.

Utilizando como definição para a entropia do sistema

$$
S(\rho)=-\ln \left(\int|\rho(\gamma)|^{2} d \gamma\right)
$$

e levando em conta (2.33) temos que

$$
\Delta S=S\left(\rho_{\alpha}\right)-S(\rho)=-\ln \left(\frac{\int\left|\rho_{\alpha}(\gamma)\right|^{2} d \gamma}{\int|\rho(\gamma)|^{2} d \gamma}\right) \geq 0
$$

e segundo [16], a heterogeneidade distribucional no espaço de fases é definida como

$$
\begin{aligned}
h_{c}(\rho) \stackrel{(1)}{=} \lim _{\alpha \rightarrow 0} \frac{\Delta S}{\alpha} \\
\stackrel{(2)}{=} \sum_{i=1}^{2 n} \frac{\int\left|\partial \rho(\gamma) / \partial \gamma_{i}\right|^{2} d \gamma}{\int|\rho(\gamma)|^{2} d \gamma} .
\end{aligned}
$$

Segundo $\mathrm{Gu}$ [16], esta definição da heterogeneidade de $\rho$ implica na existência de um comprimento característico no espaço de fases, a saber $1 / \sqrt{h_{c}(\rho)}$, que mede o comprimento de onda médio $^{3}$ das oscilações da densidade $\rho$. Como mencionado anteriormente, podemos ver na equação (2.41) que as quantidades $h_{c}(\rho)$ e $\chi_{c}^{2}(t)$ são equivalentes (ver (2.13)).

\footnotetext{
${ }^{3} \mathrm{O}$ termo médio aqui não deve ser confundido com a média devida ao coarse-graining.
} 


\subsubsection{Contexto Quântico}

O análogo quântico da heterogeneidade distribuicional no espaço de fases, definida em termos do operador densidade, pode ser obtido de através do mesmo procedimento seguido no contexto clássico. Consideremos um sistema quântico com $n$ graus de liberdade, representado por um operador densidade $\hat{\rho}$. Seguindo [16], definimos a média (coarse-graining) sobre este operador densidade como sendo o processo pelo qual obtemos $(\varkappa=\hbar \text {, ver nota de rodapé } 1)^{4}$

$$
\hat{\rho}_{\alpha}=\int \delta_{\alpha}(\gamma) e^{\mathrm{i}(\gamma \hat{\gamma})} \hat{\rho} e^{-\mathrm{i}(\gamma \hat{\gamma})} d \gamma,
$$

onde $\lim _{\alpha \rightarrow 0} \hat{\rho}_{\alpha}=\hat{\rho}, \delta_{\alpha}(\gamma)$ representa a função de alisamento utilizada em (2.33) e $\hat{\gamma}_{j}=-\hat{p}_{j}$ e $\hat{\gamma}_{j+n}=\hat{q}_{j}, j=1, \ldots, n$, são os $2 n$ operadores associados as coordenadas no espaço de fases $\gamma_{j} \equiv\left(q_{j}, p_{j}\right), \operatorname{com} j=1, \ldots, 2 n$.

Utilizando para a entropia do sistema, novamente seguindo [16], a seguinte expressão

$$
S(\hat{\rho})=-\ln \left(\operatorname{Tr}\left[\hat{\rho}^{2}\right]\right),
$$

e levando em conta as equações (2.42) concluímos que

$$
\Delta S=S\left(\hat{\rho}_{\alpha}\right)-S(\hat{\rho})=-\ln \left(\frac{\operatorname{Tr}\left[\hat{\rho}_{\alpha}^{2}\right]}{\operatorname{Tr}\left[\hat{\rho}^{2}\right]}\right) \geqslant 0 .
$$

Introduzimos então o funcional não-negativo do operador densidade $\hat{\rho}$

$$
\begin{aligned}
h_{q}(\hat{\rho}) & \stackrel{(1)}{=} \lim _{\alpha \rightarrow 0} \frac{\Delta S}{\alpha} \\
& \stackrel{(2)}{=} \sum_{i=1}^{2 n} \frac{\int\left|\partial \rho^{W}(\gamma) / \partial \gamma_{i}\right|^{2} d \gamma}{\int\left|\rho^{W}(\gamma)\right|^{2} d \gamma} \\
& \stackrel{(3)}{=}-\sum_{j=1}^{2 n} \frac{\operatorname{Tr}\left(\left[\hat{\gamma}_{j}, \hat{\rho}\right]^{2}\right)}{\operatorname{Tr}\left(\hat{\rho}^{2}\right)},
\end{aligned}
$$

onde $\rho^{W}(\gamma)$ é a função de Wigner do operador densidade $\hat{\rho}$ dada em (2.22). A equação (2.45)(2) pode ser obtida utilizando o mesmo procedimento empregado no contexto clássico (ver Apêndice B) enquanto que a equação (2.45)(3) segue diretamente da definição de $h_{q}(\hat{\rho})$ (ver Apêndice C).

Da equação (2.45)(3) obtemos a desigualdade

$$
h_{q}(\hat{\rho}) \leq 2 \sum_{j=1}^{n} \frac{\operatorname{Tr}\left[\hat{\rho}^{2}\left(\hat{q}_{j}^{2}+\hat{p}_{j}^{2}\right)\right]}{\operatorname{Tr}\left[\hat{\rho}^{2}\right]},
$$

\footnotetext{
${ }^{4}$ Note que, devido à não comutatividade $\operatorname{dos} q_{j}$ e $p_{j}$, a definição (2.42) pode levar a diferentes definições "classicamente equivalentes".
} 
que garante que $h_{q}(\hat{\rho})$ é limitada para todo ensemble que consiste de sistemas quânticos limitados. Uma vez que, segundo [16], $1 / \sqrt{h_{q}(\hat{\rho})}$ representa a escala do espaço de fases na qual flutuações apreciáveis da densidade podem ser observadas, esta escala precisa ser maior que a escala mínima dada pela relação de incerteza $\Delta q \Delta p \geq 1 / 2$

$$
\min \{\Delta q, \Delta p\} \geq 1 / 2 \mathbf{L}
$$

$\operatorname{com} \mathbf{L}=\max \{\Delta q, \Delta p\}$. Em outras palavras, devemos ter

$$
h_{q}(\hat{\rho}) \leq 4 \mathbf{L}^{2}
$$

que é compatível com a desigualdade (2.46).

Uma outra característica interessante da heterogeneidade quântica (2.45) é que esta pode ser definida mesmo para um único sistema quântico, o que não ocorre com a heterogeneidade clássica (2.41), que não possui significado para uma distribuição de um único ponto no espaço de fases. Novamente da equação (2.45)(3), é possível obter que a heterogeneidade para um sistema quântico descrito por uma função de onda $\psi$ pode ser expressa como

$$
h_{q}(\psi)=2 \sum_{j=1}^{n}\left[\left(\Delta q_{j}\right)^{2}+\left(\Delta p_{j}\right)^{2}\right]
$$

onde

$$
\begin{aligned}
& \left(\Delta q_{j}\right)^{2}=\left\langle\psi\left|\hat{q}_{j}^{2}\right| \psi\right\rangle-\left\langle\psi\left|\hat{q}_{j}\right| \psi\right\rangle^{2} \\
& \left(\Delta p_{j}\right)^{2}=\left\langle\psi\left|\hat{p}_{j}^{2}\right| \psi\right\rangle-\left\langle\psi\left|\hat{p}_{j}\right| \psi\right\rangle^{2}
\end{aligned}
$$

que é idêntica a (2.30).

Podemos resumir as principais características globais do comportamento de $h_{q}(\hat{\rho})$, pelo menos para os sistemas estudados em [16, 18, 23], da seguinte forma: (i) para sistemas classicamente regulares (integráveis), $h_{q}(\hat{\rho})$ oscila como uma função do tempo exibindo crescimento inicial como uma potência do tempo; (ii) para sistemas classicamente caóticos, $h_{q}(\hat{\rho})$ apresenta inicialmente um crescimento exponencial com o tempo, cuja taxa de crescimento assintótico é dada pelo expoente de Lyapunov máximo do sistema clássico correspondente, até atingir um patamar de saturação. Este comportamento inicial ${ }^{5}$ pode ser visto na figura 2.1, que mostra a dependência temporal de $h_{q}(\psi)$ para a versão quântica do sistema ${ }^{6}$

\footnotetext{
${ }^{5}$ É importante nota que o comportamento quântico do sistema (2.51) é na verdade periódico com um período da ordem de $\mathcal{N}[16,30]$.

${ }^{6}$ No Hamiltoniano (2.48), $\delta_{1}(t)$ representea uma sequência de funções $\delta$ de período um.
} 


$$
H=\frac{1}{2} p^{2}+\frac{1}{2} \mathcal{K} q^{2} \delta_{1}(t),
$$

para o qual a evolução temporal de uma distribuição no espaço de fases $\rho^{t}(p, q)$ é dada explicitamente por

$$
\rho^{k+1}\left(p_{k+1}, q_{k+1}\right)=\rho^{k}\left(p_{k}, q_{k}\right),
$$

com

$$
\begin{aligned}
& p_{k+1}=p_{k}-\mathcal{K} q_{k} \quad(\bmod 1) \\
& q_{k+1}=q_{k}+p_{k+1} \quad(\bmod 1) .
\end{aligned}
$$

Esta versão quântica é dada por [31]

$$
\hat{\rho}^{k+1}=\hat{\mathcal{D}} \hat{\rho}^{k} \hat{\mathcal{D}}^{\dagger}
$$

onde

$$
\hat{\mathcal{D}}=\exp \left(-\mathrm{i} \pi \mathcal{N} \hat{p}^{2}\right) \exp \left(-\mathrm{i} \pi \mathcal{N} \mathcal{K} \hat{q}^{2}\right) .
$$

Na figura 2.1 os casos A, B e C referem-se respectivamente aos estados iniciais

$$
\begin{aligned}
& \text { (A) }|\psi(0)\rangle=C \sum_{j=-s}^{s-1} \exp \left(-\frac{\pi j^{2}}{\mathcal{N}}\right)|j\rangle, \\
& \text { (B) }|\psi(0)\rangle=\frac{1}{\sqrt{2 r+1}} \sum_{j=-r}^{r}|j\rangle, \\
& \text { (C) }|\psi(0)\rangle=\frac{1}{\sqrt{2 \mathcal{N}}} \sum_{j=-s}^{s-1}\left[1+(-1)^{j}\right]|j\rangle,
\end{aligned}
$$

onde $\mathcal{N}=20000, s=10000, r=100$ e $|j\rangle, j=-s, \ldots, s-1$ são $\mathcal{N}$ autoestados do operador $\hat{q}$ com autovalores $j / \mathcal{N}$. 


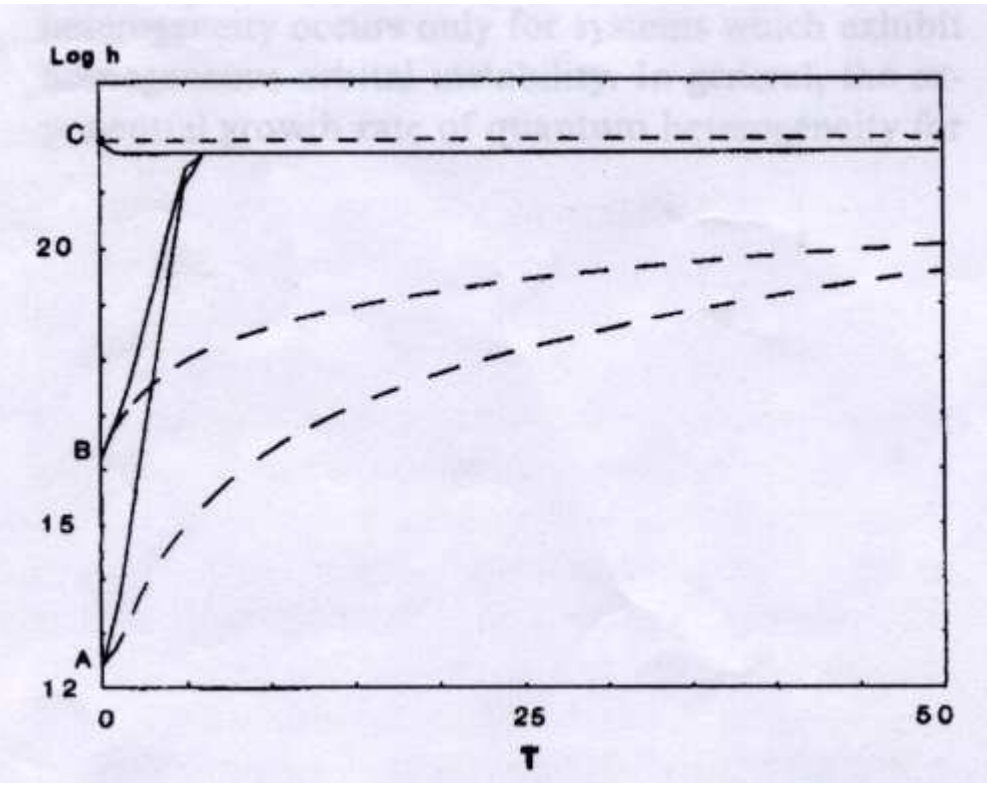

Figura 2.1: (Figura retirada de [16]). Evolução temporal de $\ln \left[h_{q}(\psi)\right]$ para diferentes estados quânticos iniciais. As curvas sólidas referem-se a $\mathcal{K}=-1$ (comportamento caótico) e as curvas tracejadas referem-se a $\mathcal{K}=0$ (comportamento regular).

Ainda com relação a equação (2.45)(3), também é importante notar que, através de uma manipulação algébrica simples, é possível mostrar que esta equivale à expressão alternativa de $\chi_{q}^{2}$ dada em (2.29). As similaridades entre $\chi_{q}^{2}$ e $h_{q}(\hat{\rho})$ também foram estudadas com algum detalhe em [23].

A utilização destas medidas da estrutura de distribuições no espaço de fases, bem como do processo de média (coase-graining) para o estudo da correspondência quântico-clássica em sistemas classicamente caóticos, já foi feita para diversos sistemas (ver por exemplo [25, 26, 32, 24, 31], e referências lá citadas). No entanto, é importante enfatizar que a utilização desta caracterização do comportamento classicamente caótico em termos de distribuições no espaço de fases, mesmo no caso de sistemas quânticos, foi feita apenas para sistemas descritos por variáveis contínuas num espaço de dimensão infinita.

Contudo, como ficará evidente mais adiante (mais precisamente no capítulo 4), para tratarmos os sistemas com propensão coletiva será necessário extender esta descrição para o caso de sistemas quânticos descritos por variáveis discretas num espaço de Hilbert que possui dimensão finita. Com este objetivo, apresentaremos no próximo capítulo o formalismo necessário para descrever tais sistemas quânticos. Além disso, mostraremos o ponto de partida de como este formalismo será utilizado (nos capítulos 4 e 5) para implementarmos os métodos descritos aqui no capítulo 2 (mais especificamente (2.45)) para estes sistemas. 


\section{Capítulo 3}

\section{Espaços de Dimensão Finita}

Neste capítulo apresentaremos o formalismo necessário à descrição de sistemas num espaço de dimensão finita. Inicialmente, introduziremos uma representação proposta por Schwinger, obtida através da simples existência de uma base ortonormal no espaço de Hilbert do sistema correspondente, na qual os graus de liberdade do sistema quântico são descritos por meio de operadores unitários no espaço de operadores correspondente. Através desta representação é possível construir "análogos discretos" de quantidades que antes eram usualmente definidas para sistemas contínuos num espaço de dimensão infinita. Mostraremos que uma das principais vantagens desta "representação discreta", é que no limite em que a dimensão do espaço se torna infinita recuperamos os resultados usuais para sistemas contínuos num espaço de dimensão infinita. Em seguida, faremos uso desta representação para implementar a descrição em termos de distribuições, apresentada no capítulo 2 (mais especificamente (2.45)), num espaço de fases de dimensão finita. Estas ferramentas serão de fundamental importância para que possamos aplicar uma descrição em termos de distribuições no espaço de fases, aos sistemas com propensão coletiva.

\subsection{Base de Operadores Unitários de Schwinger}

É um fato bem conhecido que para a descrição quântica completa de um sistema físico, é necessária a existência de um conjunto de operadores que permita a construção de todas as possíveis quantidades dinâmicas relacionadas a este sistema. Os elementos deste conjunto são então identificados como sendo os elementos de uma base completa no espaço de operadores do sistema em questão.

Um conjunto particular de operadores foi proposto por Schwinger [33, 34, 35, 36]. Em seus trabalhos, Schwinger notou que é possível obter uma base completa no espaço dos operadores a partir de um par de operadores unitários $\hat{U}$ e $\hat{V}$, que atuam no conjunto de $D$ autovetores um do outro, da seguinte forma:

$$
\hat{V}^{s}\left|u_{n}\right\rangle=\left|u_{n-s}\right\rangle, \quad \hat{U}^{s}\left|v_{n}\right\rangle=\left|v_{n+s}\right\rangle, \quad n=0,1, \ldots, D-1
$$


onde temos subentendida a condição cíclica

$$
\left|u_{k}\right\rangle \equiv\left|u_{k(\bmod D)}\right\rangle, \quad\left|v_{m}\right\rangle \equiv\left|v_{m(\bmod D)}\right\rangle
$$

que implica que

$$
\hat{U}^{D}=\hat{V}^{D}=\hat{1}
$$

e de onde vem que os autovalores de $\hat{U}$ e $\hat{V}$ são raízes da unidade, ou seja,

$$
\hat{U}\left|u_{k}\right\rangle=\exp \left[\frac{2 \pi \mathrm{i}}{D} k\right]\left|u_{k}\right\rangle, \quad \hat{V}\left|v_{k}\right\rangle=\exp \left[\frac{2 \pi \mathrm{i}}{D} k\right]\left|v_{k}\right\rangle
$$

o que mostra que o par de operadores $\hat{U}$ e $\hat{V}$ obedece também a uma álgebra de Weyl:

$$
\hat{U}^{j} \hat{V}^{l}=\exp \left[\frac{2 \pi \mathrm{i}}{D} j l\right] \hat{V}^{l} \hat{U}^{j}
$$

e seus autovetores são relacionados por uma transformada de Fourier discreta:

$$
\left\langle v_{k} \mid u_{n}\right\rangle=\frac{1}{\sqrt{D}} \exp \left[-\frac{2 \pi \mathrm{i}}{D} k n\right] .
$$

Vale a pena mencionar o fato de que esta construção é totalmente geral, uma vez que Schwinger obteve os resultados acima a partir da simples suposição da existência de $D$ estados ortonormais (qualquer base ortonormal em um espaço vetorial complexo de dimensão $D$ ). Schwinger então, verificou que o par de operadores $\hat{U}$ e $\hat{V}$ poderia ser utilizado para definir uma base no espaço dos operadores (como será discutido com maiores detalhes na próxima seção) e que a partir desta base seria possível obter uma descrição completa do sistema físico em questão.

Como mencionado anteriormente, uma das principais vantagens desta representação de Schwinger é o fato de podermos gerar os resultados usuais para um espaço de dimensão infinita no limite $D \rightarrow \infty$. Afim de verificarmos esta propriedade de uma forma explícita, seguindo [6], primeiro introduzimos o fator de rede

$$
\epsilon=\sqrt{\frac{2 \pi}{D}}
$$

que obviamente se anula no limite $D \rightarrow \infty$. Em seguida construímos, a partir dos autoestados de $\hat{U}$ e $\hat{V}$, dois operadores hermitianos $\hat{P}$ e $\hat{Q}$ de tal forma que

$$
\hat{P}=\sum_{j=-(D-1) / 2}^{(D-1) / 2} j \epsilon^{\delta} p_{0}\left|v_{j}\right\rangle\left\langle v_{j}\left|, \quad \hat{Q}=\sum_{j^{\prime}=-(D-1) / 2}^{(D-1) / 2} j^{\prime} \epsilon^{2-\delta} q_{0}\right| u_{j^{\prime}}\right\rangle\left\langle u_{j^{\prime}}\right|,
$$

onde $\delta$ é um parâmetro livre que pode assumir qualquer valor dentro do intervalo aberto $(0,2)$ (a discussão original de Schwinger é equivalente a assumir $\delta=1$ ). Os parâmetros $p_{0}$ e $q_{0}$ são 
parâmetros reais que podem carregar unidades apropriadas de acordo com o sistema físico em questão (momento e posição por exemplo), e $\Delta p=\epsilon^{\delta} p_{0}$ e $\Delta q=\epsilon^{2-\delta} q_{0}$ são as distâncias entre autovalores sucessivos dos operadores $\hat{P}$ e $\hat{Q}$, respectivamente.

Com o auxílio dos operadores $\hat{P}$ e $\hat{Q}$, podemos reescrever os operadores de Schwinger $\hat{U}$ e $\hat{V}$

$$
\hat{V}=\exp \left[\frac{\mathrm{i} \epsilon^{2-\delta} \hat{P}}{p_{0}}\right], \quad \hat{U}=\exp \left[\frac{\mathrm{i} \epsilon^{\delta} \hat{Q}}{q_{0}}\right]
$$

de modo que os autoestados de $(\hat{U}, \hat{V})$ e $(\hat{P}, \hat{Q})$ se relacionam da seguinte forma

$$
\left|v_{j}\right\rangle \equiv|p\rangle, \quad\left|u_{j^{\prime}}\right\rangle \equiv|q\rangle,
$$

com $q=q_{0} \epsilon^{2-\delta} j^{\prime}$ e $p=p_{0} \epsilon^{\delta} j$. Consequentemente(3.8) pode ser reescrita como

$$
\hat{P}=\frac{1}{\epsilon^{\delta} p_{0}} \sum_{p=-[(D-1) / 2] \epsilon^{\delta} p_{0}}^{[(D-1) / 2] \epsilon^{\delta} p_{0}} p|p\rangle\left\langle p\left|\Delta p, \quad \hat{Q}=\frac{1}{\epsilon^{2-\delta} q_{0}} \sum_{q=-[(D-1) / 2] \epsilon^{2-\delta} q_{0}}^{[(D-1) / 2] \epsilon^{2-\delta} q_{0}} q\right| q\right\rangle\langle q| \Delta q
$$

e as equações (3.1) se tornam

$$
\exp \left[\frac{\mathrm{i} q^{\prime} \hat{P}}{p_{0} q_{0}}\right]|q\rangle=\left|q-q^{\prime}\right\rangle, \quad \exp \left[\frac{\mathrm{i} p^{\prime} \hat{Q}}{p_{0} q_{0}}\right]|p\rangle=\left|p+p^{\prime}\right\rangle
$$

com $\left(p^{\prime}, q^{\prime}\right)$ definidos segundo (3.10).

As equações (3.12) acima possuem uma clara semelhança com as equações canônicas usuais para posição e momento, a menos do fato de que somente valores discretos dos parâmetros são permitidos e que as condições cíclicas (equação (3.2)) continuam válidas.

Se tomarmos agora o limite $D \rightarrow \infty, \operatorname{com} \delta$ podendo assumir qualquer valor dentro do intervalo aberto $(0,2)$, cada operador hermitiano definido pelas equações (3.11), passara a ter um espectro contínuo e não-limitado. Isto ocorre porque o limite $D \rightarrow \infty$ de $(3.11)$ nos fornece

$$
\hat{P}=\int_{-\infty}^{\infty} p|p\rangle\left\langle p\left|d p, \quad \hat{Q}=\int_{-\infty}^{\infty} q\right| q\right\rangle\langle q| d q,
$$

desde que $\Delta p \rightarrow d p$ e $\Delta q \rightarrow d q$, e as equações (3.12) são agora válidas para qualquer valor real de $\left(p, q, p^{\prime}, q^{\prime}\right)$.

Deve ser observado que, devido ao método através do qual são construídos, os parâmetros $(p, q)$ abrangem todo o conjunto dos números racionais, que é um subconjunto próprio do conjunto dos números reais. Por outro lado, todo número real pode ser escrito como o limite de uma sequência infinita de números racionais. Portanto a expressão

$$
\exp \left[\frac{\mathrm{i}\left(p^{\prime}+p^{\prime \prime}+p^{\prime \prime \prime}+\cdots\right) \hat{Q}}{p_{0} q_{0}}\right]|p\rangle=\left|p+p^{\prime}+p^{\prime \prime}+p^{\prime \prime \prime}+\cdots\right\rangle
$$


pode convergir para qualquer autovalor real e seu autovetor associado.

Temos também que, após tomarmos o limite $D \rightarrow \infty$, as condições cíclicas se tornam irrelevantes. Neste caso os operadores $\hat{P}$ e $\hat{Q}$ satisfazem às seguintes relações

$$
\begin{aligned}
& \hat{Q}|q\rangle=q|q\rangle, \quad\left\langle q^{\prime} \mid q\right\rangle=\delta\left(q^{\prime}-q\right), \quad-\infty \leqslant q^{\prime}, q \leqslant \infty \\
& \hat{P}|p\rangle=p|p\rangle, \quad\left\langle p^{\prime} \mid p\right\rangle=\delta\left(p^{\prime}-p\right), \quad\langle p \mid q\rangle=\frac{1}{\sqrt{2 \pi p_{0} q_{0}}} \exp \left(\frac{\mathrm{i} p q}{p_{0} q_{0}}\right) .
\end{aligned}
$$

Estas são as já familiares relações que descrevem um par canônico usual de variáveis de posição e momento, bastando apenas que $p_{0} q_{0}=\hbar$.

Um ponto interessante que devemos notar, é que os fatores $\epsilon^{2-\delta}$ e $\epsilon^{\delta}$, de certa forma, controlam o quão "rapidamente" (à medida que $D$ aumenta) o espectro dos operadores $\hat{P}$ e $\hat{Q}$, inicialmente discreto, se torna "contínuo", ou seja, o quão "rapidamente" perde-se a capacidade de distinguir dois autovalores consecutivos. O resultado acima é portanto bastante peculiar, uma vez que ele nos mostra que a maneira como o limite é tomado não afeta o resultado final.

A situação se torna um pouco diferente se nós considerarmos o caso extremo $\delta=0$ (ou $\delta=2$, que é equivalente). Neste caso, segundo a definição dos operadores (3.8) (lembrando que em (3.8) as letras $P$ e $Q$ não necessariamente significam momento e posição, mas sim operadores genéricos cujo significado depende da escolha do parâmetro $\delta$ ), uma das variáveis não é escalonada e o que se obtém é

$$
\hat{V}=\exp \left[\frac{\mathrm{i} \epsilon^{2} \hat{M}}{m_{0}}\right], \quad \hat{U}=\exp \left[\frac{\mathrm{i} \hat{\Theta}}{\theta_{0}}\right]
$$

onde

$$
\hat{M}=\sum_{j=-(D-1) / 2}^{(D-1) / 2} j m_{0}\left|v_{j}\right\rangle\left\langle v_{j}\left|, \quad \hat{\Theta}=\sum_{j^{\prime}=-(D-1) / 2}^{(D-1) / 2} j^{\prime} \epsilon^{2} \theta_{0}\right| u_{j^{\prime}}\right\rangle\left\langle u_{j^{\prime}}\right| .
$$

Novamente temos que os parâmetros $m_{0}$ e $\theta_{0}$ são os responsáveis por carregar as unidades dimensionais dos autovalores dos operadores, no caso $\hat{M}$ e $\hat{\Theta}$, e a relação entre os autoestados de $(\hat{U}, \hat{V})$ e $(\hat{M}, \hat{\Theta})$ é dada por

$$
\left|v_{j}\right\rangle \equiv|m\rangle, \quad\left|u_{j^{\prime}}\right\rangle=|\theta\rangle
$$

onde $\theta=\theta_{0} \epsilon^{2} j^{\prime}$ e $m=m_{0} j$. Neste caso, os operadores (3.18) ficam 


$$
\hat{M}=\sum_{j=-(D-1) / 2}^{(D-1) / 2} m|m\rangle\left\langle m\left|, \quad \hat{\Theta}=\frac{1}{\epsilon^{2} \theta_{0}} \sum_{j^{\prime}=-[(D-1) / 2] \epsilon^{2} \theta_{0}}^{[(D-1) / 2] \epsilon^{2} \theta_{0}} \theta\right| \theta\right\rangle\langle\theta| \Delta \theta,
$$

que no limite $D \rightarrow \infty$ nos fornece

$$
\begin{gathered}
\hat{M}=\sum_{m=-\infty}^{\infty} m|m\rangle\left\langle m\left|, \quad \hat{\Theta}=\int_{-\pi}^{\pi} \theta\right| \theta\right\rangle\langle\theta| d \theta ; \\
\hat{\Theta}|\theta\rangle=\theta|\theta\rangle, \quad\left\langle\theta^{\prime} \mid \theta\right\rangle=\delta\left(\theta^{\prime}-\theta\right), \quad-\pi \leqslant \theta^{\prime}, \theta \leqslant \pi ; \\
\hat{M}|m\rangle=m|m\rangle, \quad\left\langle m^{\prime} \mid m\right\rangle=\delta_{m^{\prime}, m}, \quad-\infty \leqslant m^{\prime}, m \leqslant \infty ; \\
\langle\theta \mid m\rangle=\frac{1}{\sqrt{2 \pi m_{0} \theta_{0}}} \exp \left(\frac{\mathrm{i} \theta m}{m_{0} \theta_{0}}\right) .
\end{gathered}
$$

Uma vez que no limite $D \rightarrow \infty$ o índice $m$ torna-se não-limitado, a notação cíclica torna-se sem significado para os estados $|m\rangle$ neste limite. Para os estados $|\theta\rangle$, no entanto, ela representa as condições de contorno usuais que um conjunto de estados de ângulo deve satisfazer, ou seja,

$$
|\theta\rangle \equiv|\theta(\bmod 2 \pi)\rangle
$$

e a atuação do operador de deslocamento ângular obedece naturalmente à essas condições de contorno. No entanto é necessário mencionar que os valores do índice $\theta$ estão confinados ao intervalo $[0,2 \pi)$, por definição.

Consequentemente, fazendo $m_{0} \theta_{0}=\hbar$ nas equações de (3.20) a (3.23), obtemos os resultados usuais para variáveis de ângulo e momento angular. Vale notar que estes foram obtidos a partir da mesma "origem discreta", da qual foram obtidos os resultados para posição e momento. Ou seja, obtivemos a apartir de um grau de liberdade que não possui equivalente clássico (ou seja, descrito por um número finito de estados), os resultados para um grau de liberdade que possui um número infinito de estados distribuídos de forma contínua. Isto nos mostra o quão versátil é a descrição de Schwinger em termos dos operadores unitários $\hat{U}$ e $\hat{V}$.

Na próxima seção mostraremos com é possível, utilizando os operadores unitários $\hat{U}$ e $\hat{V}$, construir uma base no espaço de operadores, capaz de descrever todas as quantidades de interesse físico relacionadas ao sistema em estudo.

\subsection{Formalismo no Espaço de Fases Discreto}

Foi mostrado na seção anterior e em [6], que as descrições quânticas usuais em termos coordenadas cartesianas e angulares, podem ser vistas como diferentes limites da formulação de Schwinger para tratar variáveis quânticas discretas.

Nosso objetivo agora é traduzir esta discussão para o contexto do espaço de fases. Esta poderia 
ser uma forma de unificar, sob a mesma estrutura, três formalismos aparentemente distintos, cada um adaptado a um tipo específico de variáveis quânticas, a saber cartesianas, angulares e discretas. Para tanto, utilizaremos uma técnica que tem como principal utilidade mapear operadores em funções reais de números inteiros e que é fortemente baseada na descrição de Schwinger.

Tendo em vista que a descrição de um grau de liberdade quântico caracterizado por um número finito de estados e consequentemente sem contrapartida clássica, em um espaço de fases discreto, pode ser feita através de uma base no espaço de operadores correspondente, consideremos a base no espaço de operadores cujos elementos são [4, 37]

$$
\hat{G}(j, l)=\frac{1}{D} \sum_{m, n=-\beta}^{\beta} \hat{U}^{m} \hat{V}^{n} \exp \left(\frac{\mathrm{i} \pi m n}{D}\right) \exp \left[-\frac{2 \pi \mathrm{i}}{D}(m j+n l)\right] \exp [\mathrm{i} \pi \phi(m+\beta, n+\beta ; D)],
$$

onde $(j, l) \in[-\beta, \beta]$, com $\beta=\frac{D-1}{2}$ e operadores $\hat{U}$ e $\hat{V}$ são os operadores unitários de Schwinger. A fase $\phi(m, n ; D)$, introduzida com o objetivo de garantir uma simetria mod $D$ nas operações envolvendo os índices da base como discutido em [37], é dada por

$$
\phi(m, n ; D)=D I_{m}^{D} I_{n}^{D}-m I_{n}^{D}-n I_{m}^{D}
$$

onde

$$
I_{k}^{D}=\left[\frac{k}{D}\right]
$$

denota a parte inteira de $k$ com relação a $D$.

As principais propriedades dos elementos (3.25) são

$$
\begin{gathered}
\operatorname{Tr}[\hat{G}(m, n)]=1 \\
\operatorname{Tr}\left[\hat{G}^{\dagger}(m, n) \hat{G}(r, s)\right]=D \delta_{m, r} \delta_{n, s} ; \\
\operatorname{Tr}\left[\hat{G}^{\dagger}(m, n) \hat{G}(u, v) \hat{G}(r, s)\right]=\sum_{a, b, c, d=-\beta}^{\beta} \frac{1}{D^{2}} \exp \left[\frac{\mathrm{i} \pi}{D}(b c-a d)\right] \times \\
\times \exp [-\mathrm{i} \pi \phi(a+c+\beta, b+d+\beta ; D)] \times \\
\times \exp \left\{\frac{2 \pi \mathrm{i}}{D}[a(m-u)+b(n-v)+c(m-r)+d(n-s)]\right\},
\end{gathered}
$$

Podemos então, com estas propriedades, utilizar os elementos (3.25) para representar todos os operadores lineares agindo no referido espaço $D$-dimensional, $D$ finito. Esta representação pode ser feita através da decomposição direta

$$
\hat{O}=\sum_{m, n} O(m, n) \hat{G}(m, n)
$$


onde o coeficiente $O(m, n)$, que fornece a representação do operador $\hat{O}$ no espaço de fases discreto [4], é dado por

$$
O(m, n)=\frac{1}{D} \operatorname{Tr}\left[\hat{G}^{\dagger}(m, n) \hat{O}\right] .
$$

Um ponto importante a ser obsevado é que (3.32) representa a transformada de Weyl discreta de um operador $[4,5,38]$. Isto significa que no caso em que $\hat{O} \equiv \hat{\rho},(3.32)$ nos fornece a representação (função) de Wigner de um estado quântico num espaço de fases discreto.

A propriedade (3.30) desempenha um papel importante na representação do produto de dois operadores no espaço discreto, que é dado por[37, 5]

$$
\begin{aligned}
&\left(\hat{O}_{1} \hat{O}_{2}\right)(m, n)=\operatorname{Tr}\left[\hat{G}(m, n) \hat{O}_{1} \hat{O}_{2}\right] \\
&=\frac{1}{D^{2}} \sum_{u, v, r, s=-\beta}^{\beta} O_{1}(u, v) O_{2}(r, s) \operatorname{Tr}\left[\hat{G}^{\dagger}(m, n) \hat{G}(u, v) \hat{G}(r, s)\right] \\
&=\frac{1}{D^{4}} \sum_{u, v, r, s=-\beta}^{\beta} \sum_{a, b, c, d=-\beta}^{\beta} O_{1}(u, v) O_{2}(r, s) \exp \left[\frac{\mathrm{i} \pi}{D}(b c-a d)\right] \times \\
& \times \exp [\mathrm{i} \pi \Phi(a, b, c, d ; D)] \exp \left\{\frac{2 \pi \mathrm{i}}{D}[a(m-u)+b(n-v)+c(m-r)+d(n-s)]\right\},
\end{aligned}
$$

onde

$$
\Phi(a, b, c, d ; D)=-\phi(a+c+\beta, b+d+\beta ; D) .
$$

O traço de um operador segue diretamente de (3.31) e (3.32)

$$
\operatorname{Tr}\left[\hat{O}_{1}\right]=\frac{1}{D} \sum_{m, n} O_{1}(m, n),
$$

enquanto o traço do produto de dois operadores pode ser obtido usando a hermiticidade dos elementos da base e a equação (3.29),

$$
\begin{aligned}
\operatorname{Tr}\left[\hat{O}_{1} \hat{O}_{2}\right] & =\frac{1}{D^{2}} \sum_{m, n=-\beta}^{\beta} \sum_{j, l=-\beta}^{\beta} O_{1}(m, n) O_{2}(j, l) \operatorname{Tr}\left[\hat{G}^{\dagger}(m, n) \hat{G}(j, l)\right] \\
& =\frac{1}{D} \sum_{m, n=-\beta}^{\beta} O_{1}(m, n) O_{2}(m, n) .
\end{aligned}
$$

Uma vez que temos a representação no espaço de fases discreto, do produto de dois operadores, torna-se simples obter a representação do comutador de dois operadores neste espaço 


$$
\begin{aligned}
& {\left[\hat{O}_{1}, \hat{O}_{2}\right] \quad(m, n)=\frac{2 \mathrm{i}}{D^{4}} \sum_{u, v, r, s=-\beta}^{\beta} \sum_{a, b, c, d=-\beta}^{\beta} O_{1}(u, v) O(r, s) \times} \\
& \times \exp [\mathrm{i} \pi \Phi(a, b, c, d ; D)] \Gamma(m, n, u, v, r, s, a, b, c, d ; D)
\end{aligned}
$$

onde

$$
\begin{aligned}
& \Gamma(m, n, u, v, r, s, a, b, c, d ; D)=\sin \left[\frac{\pi}{D}(b c-a d)\right] \times \\
& \quad \times \exp \left\{\frac{2 \pi \mathrm{i}}{D}[a(m-u)+b(n-v)+c(m-r)+d(n-s)]\right\} .
\end{aligned}
$$

Existe um interesse particular na representação no espaço discreto do comutador de dois operadores, pois com ele é possível estudar, por exemplo, a evolução temporal do operador densidade na equação de von Neumann [5]. Na próxima seção, aplicaremos as ferramentas descritas nesta seção ao formalismo de distribuições no espaço de fases descrito na seção 2.2. Mais especificamente, encontraremos um "análogo discreto" da heterogeneidade distribucional no espaço de fases (2.45).

\subsection{Distribuições num Espaço de Fases Discreto}

Nesta seção, aplicaremos o formalismo no espaço discreto apresentado na seção anterior com o objetivo de extendermos o estudo da estrutura de distribuições no espaço de fases a sistemas caracterizados por espaços discretos. Para tanto, utilizaremos a representação de Schwinger no espaço de operadores afim de obtermos um "análogo discreto" de (2.45), que no limite $D \rightarrow \infty$ nos forneça os resultados conhecidos para as representações canônicas (por exemplo (2.45) para $p$ e $q$ ).

Definimos então, para um sistema $D$-dimensional ( $D$ finito) e seguindo [31, 22], o "análogo discreto" do operador densidade com média (coarse-graining) descrito em (2.42), como sendo ${ }^{1}$

$$
\hat{\rho}_{\alpha}=\frac{1}{M} \sum_{m, n} \Delta_{\alpha}(m, n) \hat{U}^{m} \hat{V}^{n} \hat{\rho} \hat{V}^{\dagger n} \hat{U}^{\dagger m},
$$

onde $\hat{\rho}$ é o operador densidade sem média e $\Delta_{\alpha}(m, n)$ é uma versão discreta para a função de alisamento em (2.42), que agora depende das variáveis discretas $(m, n) \in[-(M-1) / 2,(M-1) / 2]$, com $M$ ímpar. De (3.39) e fazendo uma analogia com (2.42), para que tenhamos $\lim _{\alpha \rightarrow 0} \hat{\rho}_{\alpha}=\hat{\rho}$, vemos que a função $\Delta_{\alpha}(m, n)$ deve possuir o seguinte comportamento assintótico

$$
\lim _{\alpha \rightarrow 0} \Delta_{\alpha}(m, n)=M \delta_{m, 0} \delta_{n, 0}
$$

\footnotetext{
${ }^{1}$ Uma generalização para vários graus de liberdade, em termos de uma decomposição em fatores primos da dimensão do espaço de Hilbert do sistema, pode ser feita segundo proposto por Schwinger[33]. Esta propriedade da dimensão finita do espaço foi estudada de forma rigorosa em [39].
} 
Além disso, temos que $\Delta_{\alpha}(m, n)$ satisfaz à seguinte condição de normalização

$$
\sum_{m, n} \Delta_{\alpha}(m, n)=1
$$

É fácil verificar que no limite $M \rightarrow \infty(3.39)$ se torna (2.42). Para tanto, tomemos $M=D$ e consideremos as definições $(3.7)$ a $(3.10)^{2}$. Através da mudança de variáveis $u=p_{0} \epsilon^{\delta} m$ e $v=$ $-q_{0} \epsilon^{2-\delta} n$, é possível mostrar que (3.39) fica

$$
\hat{\rho}_{\alpha}=\frac{1}{q_{0} p_{0} \epsilon^{2} D} \sum_{u, v=-\beta}^{\beta} \Delta u \Delta v \Delta_{\alpha}(u, v) \exp \left[-\frac{\mathrm{i} v \hat{P}}{p_{0} q_{0}}\right] \exp \left[\frac{\mathrm{i} u \hat{Q}}{p_{0} q_{0}}\right] \hat{\rho} \exp \left[-\frac{\mathrm{i} u \hat{Q}}{p_{0} q_{0}}\right] \exp \left[\frac{\mathrm{i} v \hat{P}}{p_{0} q_{0}}\right]
$$

onde $\Delta u=p_{0} \epsilon^{\delta}$ e $\Delta v=q_{0} \epsilon^{2-\delta}$ é distância entre autovalores consecutivos.

Se tomarmos o limite $D \rightarrow \infty$, considerando que $\Delta u \rightarrow d u$ e $\Delta v \rightarrow d v$ devido ao adensamento do espectro dos operadores $\hat{P}$ e $\hat{Q}$, obtemos

$$
\hat{\rho}_{\alpha}=\frac{1}{2 \pi q_{0} p_{0}} \int_{-\infty}^{\infty} \int_{-\infty}^{\infty} d u d v \delta_{\alpha}(u, v) \exp \left[-\frac{\mathrm{i} v \hat{P}}{p_{0} q_{0}}\right] \exp \left[\frac{\mathrm{i} u \hat{Q}}{p_{0} q_{0}}\right] \hat{\rho} \exp \left[-\frac{\mathrm{i} u \hat{Q}}{p_{0} q_{0}}\right] \exp \left[\frac{\mathrm{i} v \hat{P}}{p_{0} q_{0}}\right]
$$

e utilizando a relação,

$$
\exp \left[-\mathrm{i}\left(v \frac{\hat{P}}{p_{0} q_{0}}-u \frac{\hat{Q}}{p_{0} q_{0}}\right)\right]=\exp \left(-\mathrm{i} v \frac{\hat{P}}{p_{0} q_{0}}\right) \exp \left(\mathrm{i} u \frac{\hat{Q}}{p_{0} q_{0}}\right) \exp \left(\mathrm{i} \frac{u v}{2 p_{0} q_{0}}\right)
$$

temos que (3.43) se torna

$$
\hat{\rho}_{\alpha}=\frac{1}{2 \pi p_{0} q_{0}} \int_{-\infty}^{\infty} \int_{-\infty}^{\infty} d u d v \delta_{\alpha}(u, v) \exp \left[-\mathrm{i}\left(v \frac{\hat{P}}{p_{0} q_{0}}-u \frac{\hat{Q}}{p_{0} q_{0}}\right)\right] \hat{\rho} \exp \left[\mathrm{i}\left(v \frac{\hat{P}}{p_{0} q_{0}}-u \frac{\hat{Q}}{p_{0} q_{0}}\right)\right],
$$

que a menos de uma constante é idêntica a (2.42), se fizermos $p_{0} q_{0}=1$. Este fato nos mostra que no limite $D \rightarrow \infty$, (3.39) de fato nos fornece os mesmos resultados de (2.42) (ver Apêndice A para mais detalhes).

Podemos então utilizar (3.39) afim de obter uma "versão discreta" de (2.45). Considerando a definição de entropia (2.43) e utilizando a decomposição (3.31) e a expressão (3.36) para o traço de dois operadores, obtemos que

\footnotetext{
${ }^{2}$ Deste ponto em diante, salvo menção explícita em contrário, consideraremos $M=D$.
} 
$\operatorname{Tr}\left[\hat{\rho}_{\alpha}^{2}\right]=\frac{1}{D^{2}} \sum_{m, n} \sum_{m^{\prime}, n^{\prime}} \Delta_{\alpha}(m, n) \Delta_{\alpha}\left(m^{\prime}, n^{\prime}\right) \sum_{a, b} \operatorname{Tr}\left[\hat{G}^{\dagger}(a+n, b-m) \hat{\rho}\right] \operatorname{Tr}\left[\hat{G}^{\dagger}\left(a+n^{\prime}, b-m^{\prime}\right) \hat{\rho}\right]$

onde $\hat{G}(i, j)$ é dado por (3.25), a qual podemos substituir diretamente em (2.43) e por conseguinte obter (2.45).

No entanto, utilizaremos aqui uma definição da heterogeneidade distribucional no espaço de fases que é "mais conveniente" para descrever o comportamento de distribuições num espaço de dimensão finita. Em analogia com $(2.45)(1)$ definimos

$$
\tilde{h}_{q}(\hat{\rho})=\lim _{\alpha \rightarrow 0} \frac{\Delta S}{v(\alpha)},
$$

onde $v(\alpha)$ é a variancia da média (coarse-graining). Para o caso de uma função $\Delta_{\alpha}(m, n) \mathrm{em}$ (3.39) com a forma

$$
\Delta_{\alpha}(m, n)=C_{\alpha} \exp \left[-\frac{\left(m^{2}+n^{2}\right)}{\alpha}\right]
$$

com $C_{\alpha}$ vindo da condição de normalização (3.41), que é a forma que utilizaremos nesta tese, $v(\alpha)$ é dada por

$$
v(\alpha) \equiv-\frac{1}{f(\alpha)}\left[\frac{d f(\alpha)}{d\left(\frac{1}{\alpha}\right)}\right]
$$

com

$$
f(\alpha)=\sum_{m, n} \exp \left[-\frac{\left(m^{2}+n^{2}\right)}{\alpha}\right],
$$

o que leva a

$$
v(\alpha)=\frac{\sum_{m, n}\left(m^{2}+n^{2}\right) \exp \left[-\frac{\left(m^{2}+n^{2}\right)}{\alpha}\right]}{\sum_{m, n} \exp \left[-\frac{\left(m^{2}+n^{2}\right)}{\alpha}\right]} .
$$

A definição (3.46) da heterogeneidade em termos da variancia da média $v(\alpha)$ e não em termos de $\alpha$ como em (2.41)(1) e (2.45)(1), faz-se necessária neste contexto devido a um problema de "estabilidade" de $\tilde{h}_{q}(\hat{\rho})$ no limite $\alpha \rightarrow 0$. O que ocorre neste caso, é que a diferença de entropias $\Delta S$ se anula muito rapidamente, fazendo com que a razão $\Delta S / \alpha$ se anule para um valor de $\alpha$ relativamente grande e portanto obrigando-nos a trabalhar com uma média (coarse-graining) muito "grossa" e que perde muitos detalhes da estrutura da distribuição (função de Wigner) no espaço de fases. Por outro lado, a razão $\Delta S / v(\alpha)$ é mais estável com relação ao limite $\alpha \rightarrow 0$ devido a 
uma "compensação" na rapidez com que a diferença $\Delta S$ se anula neste limite. Consequentemente, a definição da heterogeneidade (3.46) em termos de $v(\alpha)$ permite que nos aproximemos mais do valor $\alpha=0$ e portanto que trabalhemos com uma média (coarse-graining) mais "fina", conseguindo assim levar em conta mais detalhes da estrutura da distribuição (função de Wigner) no espaço de fases.

A figura 3.1 mostra o comportamento de $f(\alpha), v(\alpha)$ e $v(\alpha) / \alpha$ para $M=11$. Nesta figura, podemos ver que para $\alpha \lesssim 0.5$ a função $f(\alpha) \simeq 1$, ou seja, o limite $\alpha \rightarrow 0$ se torna inefetivo e como consequência direta a média se torna menos efetiva, o que implica $S\left(\hat{\rho}_{\alpha}\right) \simeq S(\hat{\rho})$. Nos gráficos para $v(\alpha)$ e $v(\alpha) / \alpha$, vemos que ambas se anulam para $\alpha \lesssim 0.5$ e que isto ocorre mais rapidamente para $v(\alpha)$ do que para $\alpha$, o que compensaria a inefetividade da média. Isto de fato ocorre, para $\alpha \lesssim 0.5$ temos que $S\left(\hat{\rho}_{\alpha}\right)-S(\hat{\rho})$ diminiu por inefetividade da média, mas o mesmo ocorre com $v(\alpha)$ fazendo com que $\tilde{h}_{q}(\hat{\rho})$, definida da forma $(2.45)(1)$, seja relativamente estável para $\alpha \sim 0.25$.
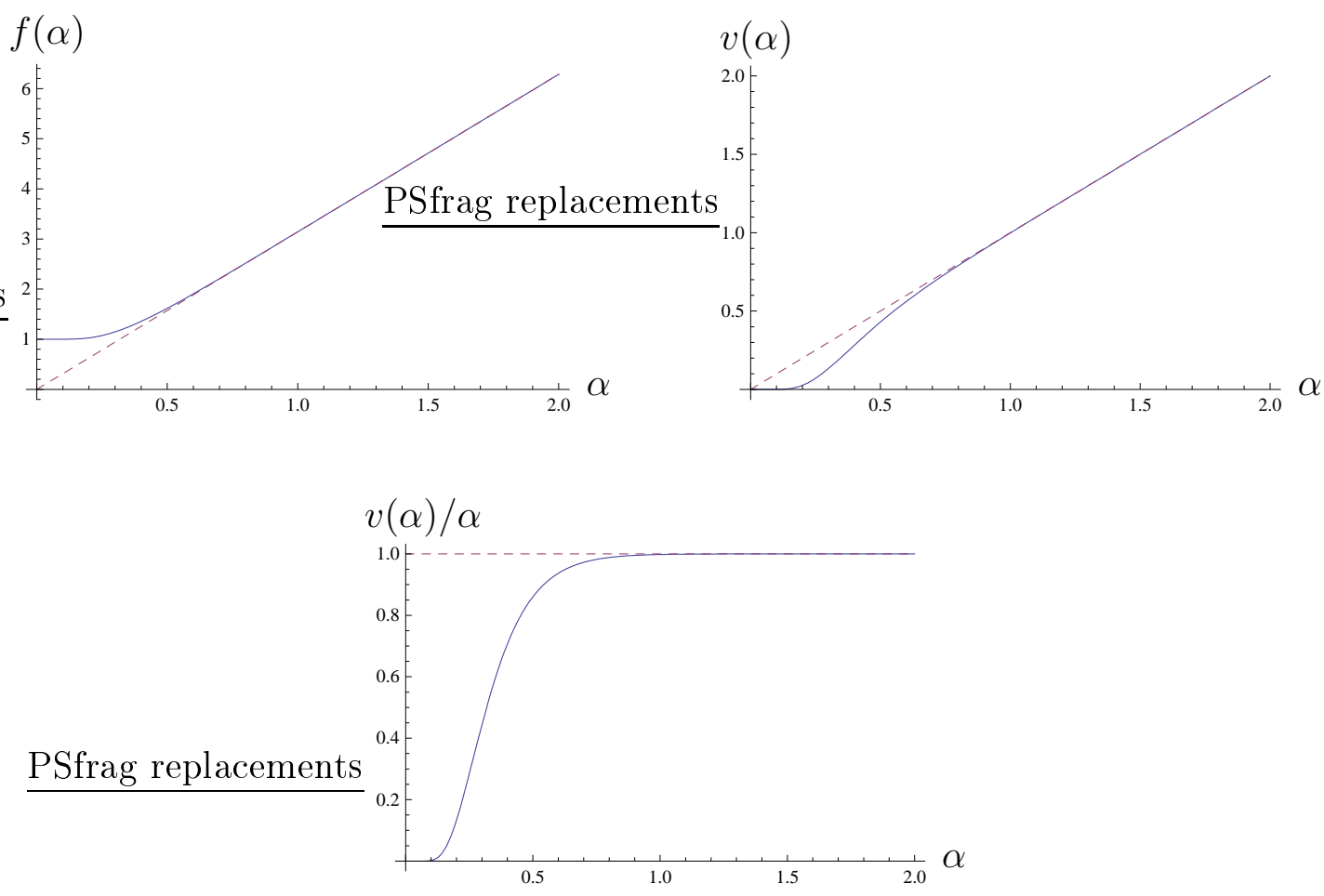

Figura 3.1: Comparação entre a função $f(\alpha)$, a variancia $v(\alpha)$ e $v(\alpha) / \alpha$ para $M=11$. As linhas tracejadas correspondem respectivamente às funções $\pi \alpha, \alpha$ e 1.

Um outro ponto importante a ser mencionado é que ao analizarmos a expressão que define $\hat{\rho}_{\alpha}$ (3.39) vemos que os limites $D \rightarrow \infty$ e $\alpha \rightarrow 0$ não comutam $^{3}$

$$
\lim _{\alpha \rightarrow 0} \lim _{D \rightarrow \infty} \frac{S\left(\hat{\rho}_{\alpha}\right)-S(\hat{\rho})}{v(\alpha)} \neq \lim _{D \rightarrow \infty} \lim _{\alpha \rightarrow 0} \frac{S\left(\hat{\rho}_{\alpha}\right)-S(\hat{\rho})}{v(\alpha)} \equiv 0
$$

\footnotetext{
${ }^{3}$ Esta não comutatividade dos limites também foi observada no estudo das propriedades espectrais de propagadores quânticos [40] e no estudo da decoerência em mapas quânticos [22].
} 
ou seja, que o limite $\alpha \rightarrow 0$ só deve ser tomado após o "limite contínuo" $D \rightarrow \infty$, pois caso contrário obteríamos uma função $\tilde{h}_{q}(\hat{\rho})$ identicamente nula (ver Apêndice A).

No próximo capítulo estudaremos um exemplo de sistema com propensão coletiva, o modelo de Bose-Hubbard com dois sítios e $N$ bósons. Este sistema, apesar de ser bem simples, possui um comportamento bastante rico e portanto servirá como um bom campo de aplicação para os resultados obtidos até aqui. 


\section{Capítulo 4}

\section{Modelo com Propensão Coletiva}

Neste capítulo aplicaremos os resultados até aqui obitdos, para um modelo que apresenta propensão coletiva, o modelo de Bose-Hubbard com dois sítios. Este modelo descreve, por exemplo, um condensado de Bose-Einstein confinado em uma armadilha do tipo poço duplo. O fato deste modelo ter sido extensivamente estudado na literatura em suas mais variadas versões, faz dele um bom teste inicial para os métodos desenvolvidos nos capítulos anteriores, ou seja, por termos um conhecimento melhor das características do modelo, podemos também ter um controle maior sobre a influência destas características no comportamento de $\tilde{h}_{q}(\hat{\rho})$.

\subsection{Modelo de Bose-Hubbard com Dois Sítios e $N$ Bósons}

O modelo de Bose-Hubbard descreve de maneira simplificada um sistema de bósons interagentes distribuídos em uma rede. Uma característica importante deste modelo é que este considera apenas um estado por cada sítio da rede. O modelo de Hubbard foi concebido originalmente como um modelo fundamental da Física do Estado Sólido, onde ele é mais usualmente empregado em sua versão fermiônica afim de descrever o comportamento de elétrons em sólidos, no entanto, nos últimos anos este modelo vem sendo bastante utilizado no estudo de condensados de Bose-Einstein em redes óticas.

Além disso, temos que devido às temperaturas extremamente baixas e à periodicidade precisa das redes óticas, estes sistemas abrem a possibilidade de realização experimental de vários modelos teóricos para sistemas de muitas partículas interagentes. Um exemplo importante deste tipo de realização experimental, é a transição entre uma fase superfluida e uma fase isolante de Mott, que foi observada em um condensado de Bose-Einstein preso em uma rede ótica tridimensional [41].

Para um número muito grande de sítios e partículas, a solução exata do modelo de BoseHubbard torna-se extremamente complicada, até mesmo numericamente. O caso com apenas dois sítios possui uma estrutura muito mais simples e pode ser usado para descrever, por exemplo, um condensado de Bose-Einstein em uma armadilha do tipo poço duplo. Isto faz com que este 
seja o caso mais estudado na literatura $[42,43,44,45,46,47]$ e portanto este será o modelo que utilizaremos.

\subsubsection{Representação em Termos de Operadores Coletivos (Quasi-Spin)}

A forma usual para o Hamiltoniano de Bose-Hubbard é (ver [3] para maiores detalhes sobre a construção do Hamiltoniano )

$$
H_{B H}=\sum_{i} \mathcal{E}_{i} a_{i}^{\dagger} a_{i}-\eta \sum_{\langle i, j\rangle}\left(a_{i}^{\dagger} a_{j}+a_{j}^{\dagger} a_{i}\right)+\sum_{i} \frac{\Lambda_{i}}{2} a_{i}^{\dagger} a_{i}^{\dagger} a_{i} a_{i},
$$

onde $\langle i, j\rangle$ denota que os sítios $i$ e $j$ são vizinhos e a dimensão do espaço de Hilbert correspondente é

$$
D=\frac{(N+L-1) !}{N !(L-1) !}
$$

para $N$ átomos e um número finito de sítios $L$. Na equação (4.1) vemos que o Hamiltoniano $H_{B H}$ possui um termo de "hopping" (proporcional a $\eta$ ), o qual está ligado ao tunelamento das partículas entre sítios diferentes. Além disso, vemos que este possui também um termo de interação de dois corpos (proporcial a $\Lambda_{i} / 2$ ) "intrasítio" e um termo de energia de um corpo (proporcial a $\mathcal{E}_{i}$ ), possivelmente diferente para cada sítio $i$.

No caso de apenas dois sítios $(L=2)$ e $N$ bósons distribuídos entre dois modos localizados, associados aos operadores de criação $a_{ \pm}^{\dagger}$, temos que $D=N+1$. Neste caso, considerando $\Lambda_{+}=$ $\Lambda_{-}=\Lambda$ e $\mathcal{E}_{+}=-\mathcal{E}_{-}=\mathcal{E} / 2$ temos que (4.1) fica

$$
H_{B H_{2}}=\frac{\mathcal{E}}{2}\left(a_{+}^{\dagger} a_{+}-a_{-}^{\dagger} a_{-}\right)-\eta\left(a_{+}^{\dagger} a_{-}+a_{-}^{\dagger} a_{+}\right)+\frac{\Lambda}{2}\left(a_{+}^{\dagger} a_{+}^{\dagger} a_{+} a_{+}+a_{-}^{\dagger} a_{-}^{\dagger} a_{-} a_{-}\right)
$$

onde o número total de bósons $\hat{N}=a_{+}^{\dagger} a_{+}+a_{-}^{\dagger} a_{-}$é uma constante de movimento.

Uma maneira conveniente de trabalhar com o Hamiltoniano (4.3), consiste em definir os operadores coletivos (também conhecidos na literatura como operadores de quasi-spin [2])

$$
J_{ \pm}=J_{1} \pm \mathrm{i} J_{2} \equiv a_{ \pm}^{\dagger} a_{\mp}, \quad J_{3} \equiv \frac{a_{+}^{\dagger} a_{+}-a_{-}^{\dagger} a_{-}}{2}, \quad J \equiv \frac{a_{+}^{\dagger} a_{+}+a_{-}^{\dagger} a_{-}}{2}=\frac{\hat{N}}{2}
$$

que formam a já conhecida realização de Schwinger de uma álgebra de momento angular em termos de dois modos bosônicos [48]. O papel desempenhado pelo operador $J$ é dado pela relação

$$
J_{1}^{2}+J_{2}^{2}+J_{3}^{2}=J(J+1)=\frac{\hat{N}}{2}\left(\frac{\hat{N}}{2}+1\right),
$$

ou seja, o valor do quasi-spin $J$ é a metade do número de bósons. Temos então que o Hamiltoniano 
(4.3) em termos dos operadores coletivos (4.4) fica

$$
H_{B H_{2}}=\mathcal{E} J_{3}-2 \eta J_{1}+\Lambda\left[J(J-1)+J_{3}^{2}\right] .
$$

A representação em termos de operadores coletivos, que explicita a propensão coletiva do sistema ${ }^{1}$, incluindo o fato de o operador $J(J+1)$ ser uma constante de movimento, torna o problema "solúvel", no sentido de que é possível obter os autovalores e autovetores do sistema (4.6), diagonalizando (numericamente) matrizes finitas. Para tanto utilizaremos os autovetores de $H_{B H_{2}} / N$ na seguinte representação no espaço de Fock

$$
H_{B H_{2}}\left|E_{i}\right\rangle=E_{i}\left|E_{i}\right\rangle, \quad\left|E_{i}\right\rangle=\sum_{m=-J}^{J} c_{m}^{(i)}|m\rangle_{+} \otimes|N-m\rangle_{-},
$$

que nos fornece diretamente, através dos $2 J+1=N+1$ números $\left\{\left|c_{m}^{(i)}\right|^{2}\right\}$, as distribuições correspondentes à ocupação de cada um dos dois níveis localizados. Alternativamente, podemos visualizar estas distribuições através da função de Wigner discreta, calculada utilizando as amplitudes que aparecem sob a forma dos coeficientes da expansão dos autoestados de energia $\left|E_{i}\right\rangle$ na representação (4.7). Este procedimento foi utilizado em [2] e detalhadamente discutido em [21].

A figura 4.1 mostra o espectro dos autovalores de energias de $H_{B H_{2}} / N,{ }^{2}$ para $\Lambda N=-2, \eta=\mathcal{E}=$ 0.2 e $N=64$, e o número médio de partículas $\left\langle E_{i}|\hat{N}| E_{i}\right\rangle \equiv\langle\hat{N}\rangle_{i} \equiv\left\langle\hat{N}_{+}+\hat{N}_{-}\right\rangle_{i} \equiv\left\langle a_{+}^{\dagger} a_{+}+a_{-}^{\dagger} a_{-}\right\rangle_{i}$ e suas respectivas variâncias $\sigma_{i} \equiv \sqrt{\left\langle\hat{N}^{2}\right\rangle_{i}-\langle\hat{N}\rangle_{i}^{2}}$, correspondentes a cada autovalor de energia.

Um ponto interessante a ser notado na figura 4.1 é a existência de duas regiões do espectro, separadas por uma energia $\bar{E} \simeq-37,-43$ e -55 respectivamente nos gráficos (a), (b) e (c), qualitativamente diferentes. Na primeira delas $\left(E_{i}<\bar{E}\right)$ vemos que as partículas tendem a se localizar em um dos dois modos localizados $a_{ \pm}$e que, como vemos pela variância $\sigma_{i}$ pequena, os estados internos de energia $\left|E_{i}\right\rangle$ possuem um número total de partículas bem definido. Já na segunda região $\left(E_{i} \geq \bar{E}\right)$ as partículas tendem a se deslocalizar e, devido as fortes correlações entre $N_{+}$e $N_{-}$[2], os níveis internos de energia possuem uma variância $\sigma_{i}$ grande.

A existência destas duas regiões está diretamente relacionada a assimetria dos dois modos localizados $a_{ \pm}(\mathcal{E}=0.2$ na figura 4.1 ; o caso simétrico corresponde a $\mathcal{E}=0)$ e ao valor do "parâmetro de tunelamento" $\eta / \Lambda$. Este fato fica mais claro ao observarmos na figura 4.1 que a distribuição do espectro de autovalores de $H_{B H_{2}} / N$, se modifica conforme variamos o valor do parâmetro de hopping $\eta \operatorname{com} \Lambda$ fixo (compare os gráficos (a), (b) e (c)). Este efeito foi estudado com bastante detalhes em [43, 2, 49, 50] (e referências lá citadas) e pode ser visto como diretamente ligado à transição superfluido-isolante de Mott que ocorre para $L \rightarrow \infty \operatorname{com} N / L=$ constante em (4.1) e que foi experimentalmente obsevada em [41].

\footnotetext{
${ }^{1} \mathrm{O}$ termo coletivo neste caso se refere ao fato de reduzirmos a dinâmica de um sistema com $N$ partículas (4.3) à dinâmica de um sistema com apenas um grau de liberdade (4.6).

${ }^{2} \mathrm{O}$ motivo de utilizarmos o Hamiltoniano "por partícula" $H_{B H_{2}} / N$ ficará claro mais adiante, mas podemos adiantar que é com o objetivo de estudar o limite $N \rightarrow \infty$ como limite clássico.
} 

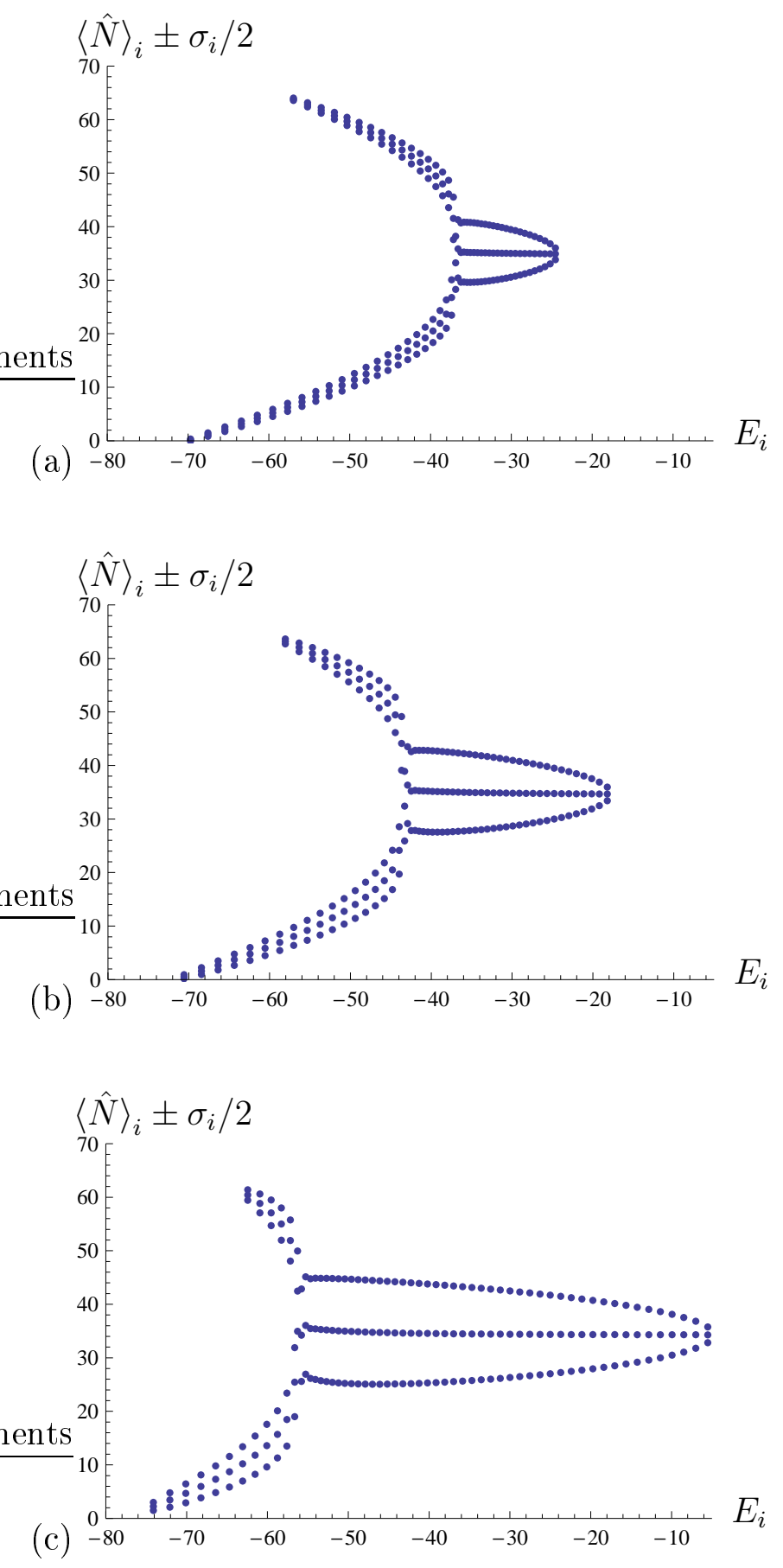

Figura 4.1: Espectro dos autovalores de energia de $H_{B H_{2}} / N$ para $\Lambda N=-2, \mathcal{E}=0.2, N=64$ e $\eta=0.1$ (a), 0.2 (b) e 0.4 (c). Também é mostrado o número médio de partículas, e sua respectiva variância, correspondente a cada autovalor de energia.

\subsubsection{Transformada de Weyl Discreta e a Função de Wigner}

Seguindo $[2,21]$, temos que para um dado estado $|a\rangle$ representado em termos da base $\left\{\left|u_{k}\right\rangle\right\}$, ou seja, 


$$
|a\rangle=\sum_{k} a_{k}\left|u_{k}\right\rangle
$$

a transformada discreta de Weyl (3.32) deste estado é dada por

$$
\begin{aligned}
D a^{W}(p, q) & =\operatorname{Tr}\left[\hat{G}^{\dagger}(p, q)|a\rangle\langle a|\right] \\
& =\frac{1}{D^{1 / 2}} \sum_{j, l} \exp \left[\frac{2 \pi}{D} \mathrm{i}(p j+q l)-\mathrm{i} \pi \phi(j, l ; D)\right] r(j, l),
\end{aligned}
$$

com

$$
r(j, l)=\frac{1}{D^{1 / 2}} \sum_{m} a_{m} a_{\{m+l\}}^{*} \exp \left[-\frac{2 \pi \mathrm{i}}{D} j\left(m+\frac{l}{2}\right)\right],
$$

onde $\{m+l\}$ denota o valor da soma $m+l$ ciclicamente confinado ao intervalo de variação adotado para os índices $m$ e $l$.

Um ponto importante que devemos notar é que as funções $a^{W}(p, q)$, construídas da forma (4.8), em geral assumem valores complexos. Afim de obter funções que assumam apenas valores reais, é necessário que o intervalo de variação dos índices discretos $j, l$ e $m$ seja simétrico com relação ao zero. Isto significa que, para $D$ ímpar, os índices $j, l$, e $m$ devem satisfazer $-(D-1) / 2 \leq$ $j, l, m \leq(D-1) / 2$, que corresponde à definição usual da transformada de Weyl contínua (ver a equação (3.8) de [4]). Neste caso temos explicitamente que

$$
\{m+l\}=m+l-D I_{m+l+(D-1) / 2}^{D},
$$

com $I_{k}^{D}$ dado por (3.27). Devemos notar também que em função do intervalo adotado para os índices $j$ e $l$ a fase $\phi(j, l ; D)$ se torna sem importância no cálculo da transformada (4.8) (ver por exemplo $[21,5])$.

As figuras 4.2(a) e 4.2(b) mostram a transformada de Weyl discreta do Hamiltoniano $H_{B H_{2}} / N$, ou seja (3.32) com $\hat{O} \equiv H_{B_{2}} / N$, e a figura 4.2(c) mostra a função de Wigner discreta (4.8) do estado fundamental de $H_{B_{2}} / N$. Em nossos cálculos numéricos adotamos unidades tais que $\hbar=1$; isto significa que energias são dadas em unidades do parâmetro de pseudopotencial de dois corpos $\Lambda$, que por sua vez é dado em unidades do parâmetro de "hopping" $\eta$ (Hz por exemplo). Neste caso, os tempos são dados em unidades do inverso de energia (ver equação (4.17)), ou seja, $1 / \Lambda$ (por exemplo $1 / \mathrm{Hz} \equiv s$ ). 

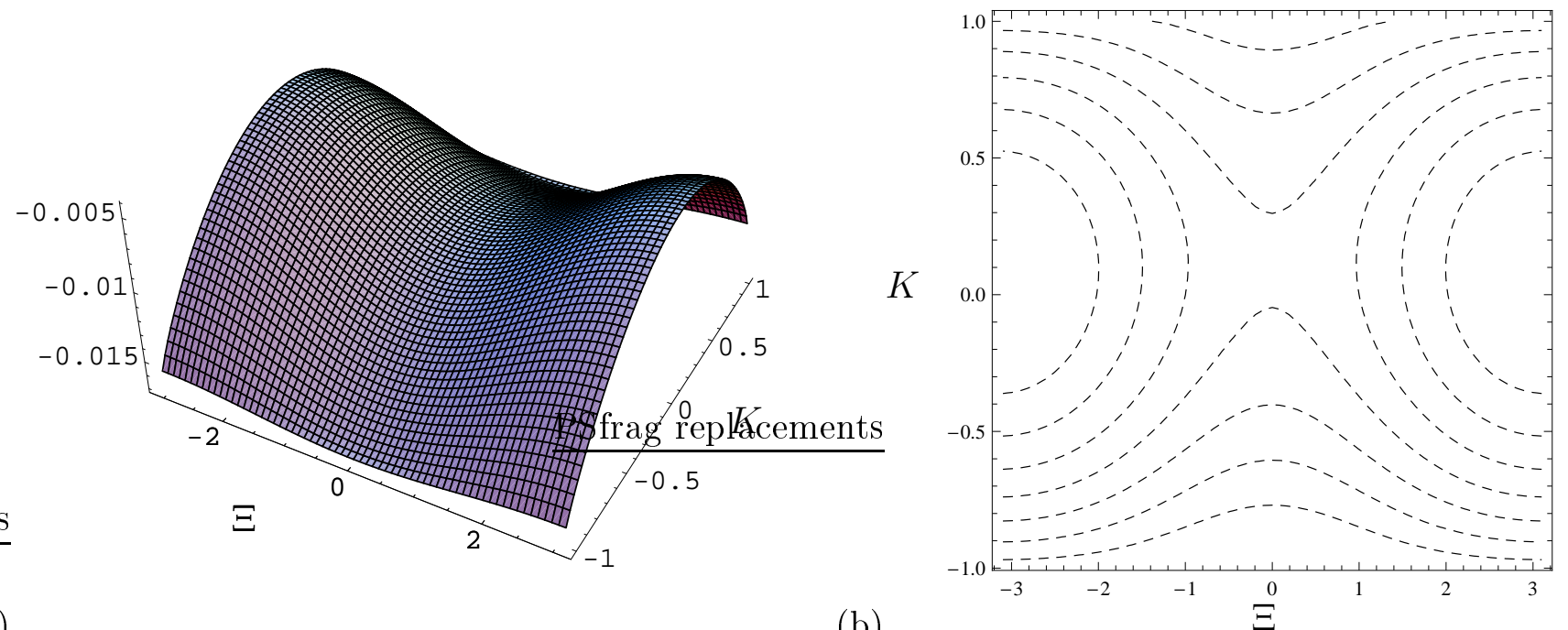

(a)

(b)

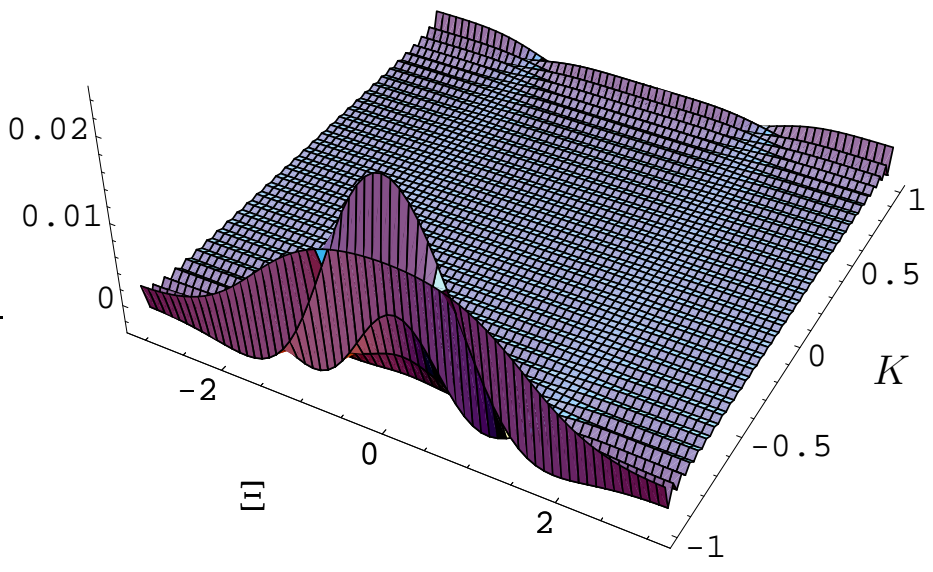

(c)

Figura 4.2: Transformada de Weyl discreta do Hamiltoniano $H_{B H_{2}} / N$ (gráficos (a) e (b)) e função de Wigner do estado fundamental de $H_{B H_{2}} / N$ (gráfico (c)) em termos das variáveis escalonadas $K \equiv q / J$ e $\Xi \equiv 2 \pi p /(2 J+1),-J \leq p, q \leq J$, que aparecem no cálculo de $\rho^{W}(p, q)$ em (4.8), para $J=N / 2=32, \mathcal{E}=\eta=0.2$ e $\Lambda N=-2$.

Como ocorre com seu equivalente para sistemas contínuos, $\rho^{W}(p, q)$ em (2.22), esta representação discreta da função de Wigner também se mostra bastante útil quando queremos visualizar explicitamente o aumento ou diminuição da estrutura no espaço de fases (neste caso discreto) e o efeito da média (coarse-graining) feita neste espaço. Isto fica claro nas figuras 4.3 a 4.6 , que mostram a transformada de Weyl discreta de um estado quântico $\left|\Psi_{t}\right\rangle$ representado por $\hat{\rho}=\left|\Psi_{t}\right\rangle\left\langle\Psi_{t}\right|$ (ver equação (4.8)), antes e após realizarmos uma média (coarse-graining) no espaço de fases, ou 
seja, a função de Wigner discreta de $\hat{\rho}$ e $\hat{\rho}_{\alpha}$ dada em (3.39). Nas figuras 4.3 a 4.6, consideramos um estado inicial $\left|\Psi_{0}\right\rangle$, localizado no espaço de fases, que possui a forma

$$
\left|\Psi_{0}\right\rangle=|\xi, \vartheta\rangle_{N} \equiv \frac{A^{\dagger N}}{\sqrt{N !}}|0\rangle
$$

cuja evolução temporal é dada por

$$
\left|\Psi_{t}\right\rangle=\sum_{n} c_{n} \mathrm{e}^{-\mathrm{i} E_{n} t / \hbar}\left|E_{n}\right\rangle
$$

$\operatorname{com} c_{n}=\left\langle E_{n} \mid \Psi_{0}\right\rangle, E_{n}$ e $\left|E_{n}\right\rangle$ sendo respectivamente os autovalores e autoestados do Hamiltoniano "por partícula" $\mathrm{H}_{\mathrm{BH}_{2}} / \mathrm{N}$.

No estado (4.9), o operador $A^{\dagger}$ é definido, em termos dos dois modos localizados $a_{ \pm}$, como

$$
A^{\dagger} \equiv \xi a_{+}^{\dagger}+\mathrm{e}^{-\mathrm{i} \vartheta} \sqrt{1-\xi^{2}} a_{-}^{\dagger}
$$

Com isso, podemos reescrever o estado (4.9) em termos dos autovetores comuns de $J(J+1)$ e $J_{3}$, que fica então

$$
|\xi, \vartheta\rangle_{N}=\sum_{n=0}^{N}\left[\sqrt{\left(\begin{array}{c}
N \\
n
\end{array}\right)}\left(\mathrm{e}^{-\mathrm{i} \vartheta} \sqrt{1-\xi^{2}}\right)^{N-n}\right]\left|J=N / 2, J_{3}=n-N / 2\right\rangle .
$$

Tais estados foram descritos em [51] como estados homogêneos (invariantes translacionais) extremais, cujo comportamento no limite $N \rightarrow \infty$ se torna totalmente clássico.

Uma propriedade interessante dos estados $|\xi, \vartheta\rangle_{N}$, é que estes coincidem com os estados coerentes de $S U(2)[52]$

$$
|N / 2, \tau\rangle \equiv \frac{1}{\left[1+|\tau|^{2}\right]^{N / 2}} \mathrm{e}^{\tau J_{+}}\left|J=N / 2, J_{z}=-N / 2\right\rangle,
$$

se fizermos

$$
\begin{gathered}
\tau \equiv \mathrm{e}^{-\mathrm{i} \vartheta} \tan \frac{\Omega}{2} \\
\xi=\cos \frac{\Omega}{2}, \quad 0 \leq \Omega \leq \pi .
\end{gathered}
$$

E como consequência direta, estes estados também satisfazem a relação de supercompleteza

$$
\frac{2 J+1}{4 \pi} \int d \Omega|N / 2, \tau\rangle\left\langle N / 2, \tau\left|=\sum_{m}\right| N / 2, m\right\rangle\langle N / 2, m|=\hat{1}_{J} .
$$

Por fim, realizamos uma média (coarse-graining) no espaço de fases, com uma "função de alisamento" $\Delta_{\alpha}(m, n)$ da forma(3.47). Numericamente, o procedimento é o seguinte: para cada 
$\hat{\rho}=\left|\Psi_{t}\right\rangle\left\langle\Psi_{t}\right|$, com $\left|\Psi_{t}\right\rangle=\sum_{r} \psi_{r}(t)\left|u_{r}\right\rangle$, calculamos os elementos de matriz $\rho^{k k^{\prime}}=\left\langle u_{k}|\hat{\rho}| u_{k^{\prime}}\right\rangle=$ $\psi_{k}^{*}(t) \psi_{k^{\prime}}(t)$ e $\rho_{\alpha}^{k k^{\prime}}=\left\langle u_{k}\left|\hat{\rho}_{\alpha}\right| u_{k^{\prime}}\right\rangle$ pela equação (3.39), com

$$
\begin{aligned}
\rho_{\alpha}^{k k^{\prime}} \stackrel{(1)}{=} \frac{1}{M} \sum_{m, n=-\frac{(M-1)}{2}}^{\frac{(M-1)}{2}} \Delta_{\alpha}(m, n)\left\langle u_{k}\left|\hat{U}^{m} \hat{V}^{n} \hat{\rho} \hat{V}^{-n} \hat{U}^{-m}\right| u_{k^{\prime}}\right\rangle \\
\stackrel{(2)}{=} \frac{C_{\alpha}}{M} \sum_{m, n=-\frac{(M-1)}{2}}^{\frac{(M-1)}{2}} \exp \left[-\frac{\left(m^{2}+n^{2}\right)}{\alpha}+\frac{2 \pi \mathrm{i}}{D} m\left(k-k^{\prime}\right)\right] \psi_{k+n}^{*}(t) \psi_{k^{\prime}+n}(t),
\end{aligned}
$$

onde $k, k^{\prime}=1, \ldots, D$ e $M$ nos diz o número de células do espaço de fases (discreto) utilizadas na média (coarse-graining). Utilizando (4.12), podemos calcular

$$
\begin{aligned}
& \operatorname{Tr}\left[\hat{\rho}^{2}\right]=\sum_{k, k^{\prime}}\left(\rho^{k k^{\prime}}\right)^{2}, \\
& \operatorname{Tr}\left[\hat{\rho}_{\alpha}^{2}\right]=\sum_{k, k^{\prime}}\left(\rho_{\alpha}^{k k^{\prime}}\right)^{2},
\end{aligned}
$$

e então obter as entropias $S(\hat{\rho}), S\left(\hat{\rho}_{\alpha}\right)$ pela equação $(2.43)$ e a heterogeneidade $\tilde{h}_{q}(\hat{\rho})$ pela equação (3.46). 

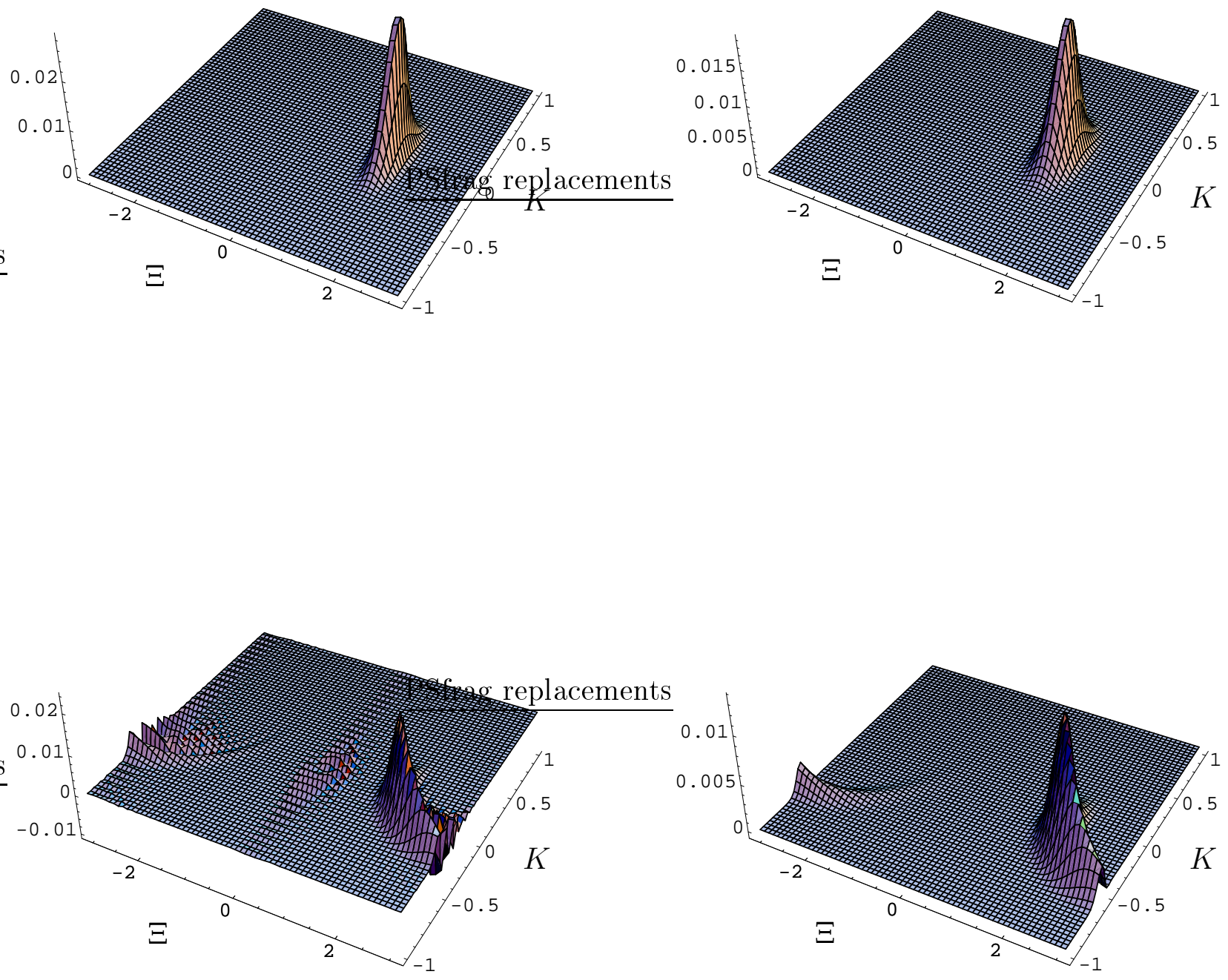

Figura 4.3: Função de Wigner discreta de $\hat{\rho}$ (esquerda) e $\hat{\rho}_{\alpha}$ (direita) em termos das variáveis $K$ e $\Xi$. Os valores dos parâmetros são $\Lambda N=-2, \eta=\mathcal{E}=0.2, N=64, M=7, K_{0}=0, \Xi_{0}=1.5$, $\alpha=4, t=0$ (acima) e $t=230$ (abaixo). 

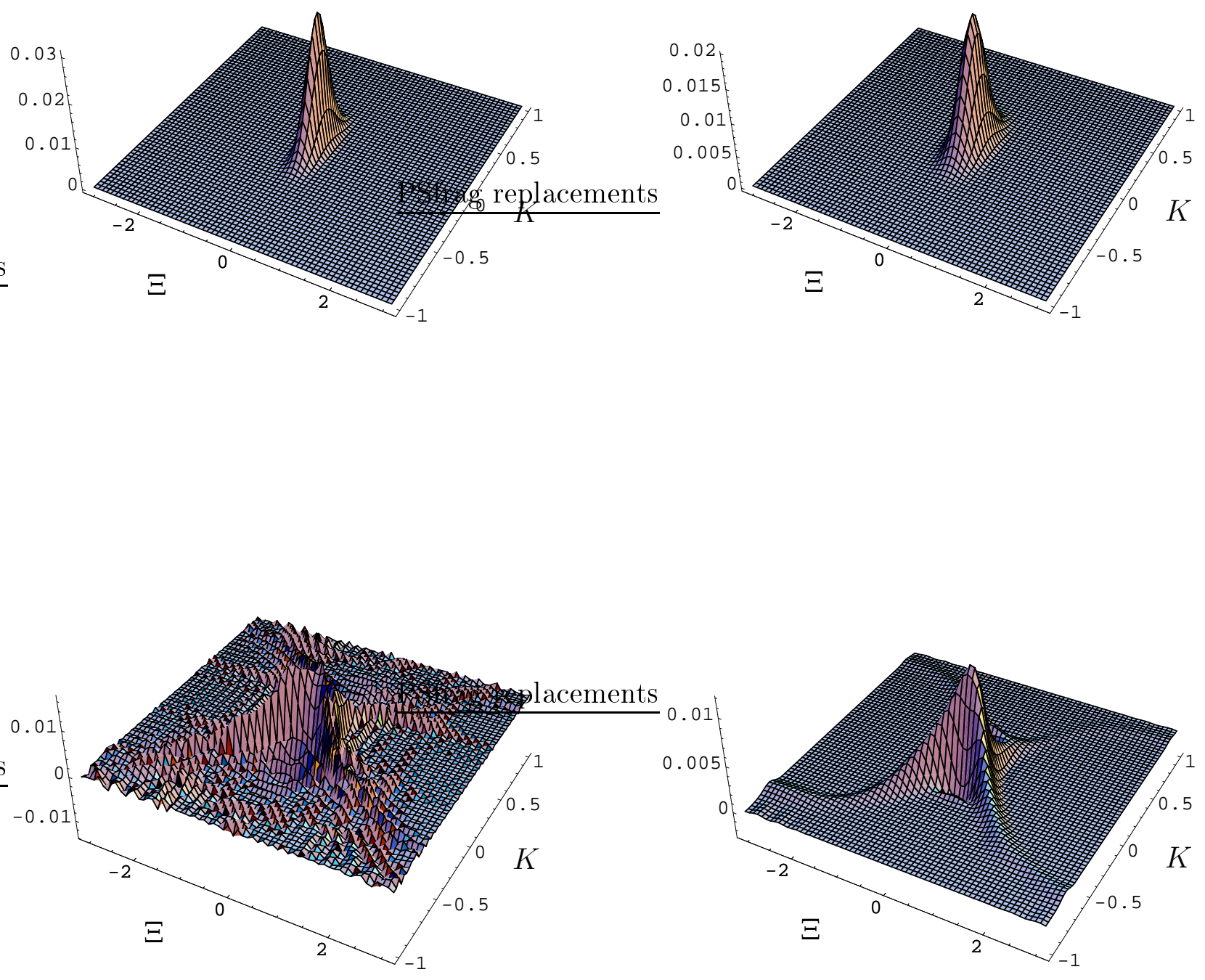

Figura 4.4: $\mathrm{O}$ mesmo da figura 4.3 para $K_{0}=0$ e $\Xi_{0}=0$. 

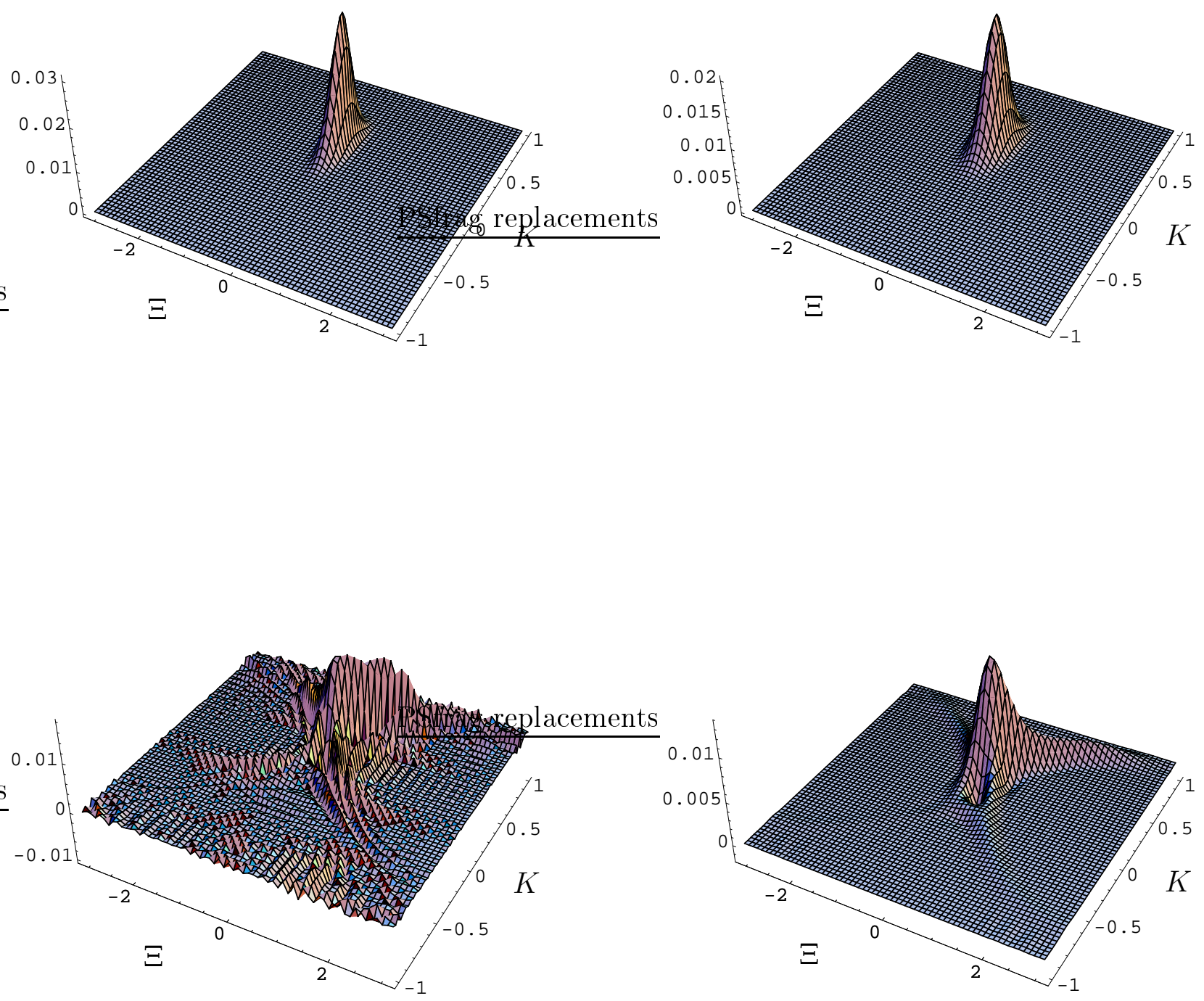

Figura 4.5: $O$ mesmo da figura 4.3 para $K_{0}=0.1, \Xi_{0}=0$. 


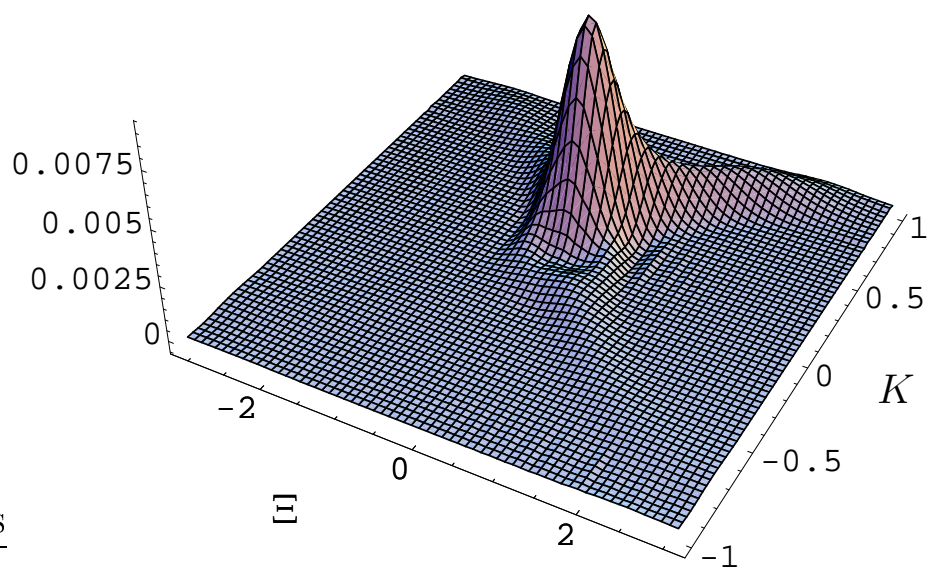

Figura 4.6: Função de Wigner discreta de $\hat{\rho}_{\alpha}$ para $\Lambda N=-2, \eta=\mathcal{E}=0.2, N=64, M=9$, $K_{0}=0.1, \Xi_{0}=0, \alpha=16$ e $t=230$.

As figuras 4.3 a 4.6 nos mostram claramente que, conforme o estado inicial evolui no tempo a função de Wigner discreta correspondente se espalha através do espaço de fases. Vemos que a forma como esta se espalha depende do ponto inicial $\left(\Xi_{0}, K_{0}\right)$, ao redor do qual o estado inicial está localizado.

Podemos ver também que para todas as condições iniciais conforme o estado inicial evolui temos o aparecimento de um padrão oscilatório (franjas) e também a ocorrência de valores negativos de $\rho^{W}(\Xi, K)$. Segundo [18, 19], este é exatamente o comportamento esperado, ou seja, a evolução da função de Wigner deve usualmente seguir a evolução de uma distribuição clássica com o acréscimo de oscilações quânticas (franjas). Nas figuras 4.3 a 4.6 também é possível visualizar o efeito da média (coarse-graining) no espaço de fases, que é principalmente o de "amortecer" estas oscilações quânticas (franjas) "alisando" (localizando) a função de Wigner no espaço de fases.

Temos então que a função de Wigner discreta (4.8), nos permite visualizar e analisar o comportamento de distribuições no espaço de fases, ainda no contexto quântico, permitindo assim uma comparação direta com o contexto clássico. Esta possibilidade abre caminho para a implementação de uma descrição em termos de distribuições no espaço de fases, mesmo no caso de um sistema com espectro puramente discreto num espaço de dimensão finita.

\subsubsection{Limite Semi-Clássico}

O sistema (4.6) possui um limite semi-clássico para $N \gg 1$ e neste caso uma formulação da dinâmica do sistema em termos clássicos se torna bastante útil. Para tanto, consideremos, ao invés 
dos operadores $J_{i}, \quad i=1,2,3$, cujos autovalores estão no intervalo entre $-J$ e $+J$, os operadores escalonados

$$
j_{i} \equiv \frac{J_{i}}{J}, \quad J=\frac{N}{2}
$$

cujos autovalores estão no intervalo entre -1 e +1 .

A definição (4.14) nos diz que o espectro dos operadores escalonados $j_{i}$ se torna cada vez mais denso à medida que nos aproximamos do regime semi-clássico $(J \rightarrow \infty)$, implicando que os $j_{i}$ podem, neste limite, ser tratados como variáveis contínuas.

Das relações de comutação de momento angular satisfeitas pelos operadores $J_{i}$, a saber

$$
\left[J_{i}, J_{j}\right]=\mathrm{i} \varepsilon_{i j k} J_{k}
$$

onde $\varepsilon_{i j k}$ é o símbolo antissimétrico, segue que

$$
\lim _{J \rightarrow \infty} \frac{J}{\mathrm{i}}\left[\frac{J_{i}}{J}, \frac{J_{j}}{J}\right] \equiv\left\{j_{i}, j_{j}\right\}=\varepsilon_{i j k} j_{k} \equiv \varepsilon_{i j k} \lim _{J \rightarrow \infty} \frac{J_{k}}{J}
$$

de tal forma que os operadores escalonados comutam no limite $J \rightarrow \infty$ e o objeto $\lim _{J \rightarrow \infty}\left(\frac{J}{\mathrm{i}}\right)\left[\frac{J_{i}}{J}, \frac{J_{j}}{J}\right]$ em (4.15) desempenha o papel dos parênteses de Poisson das variáveis dinâmicas $j_{i}$ e $j_{j}$.

O "Hamiltoniano por partícula" $H_{B H_{2}} / N$ pode então ser escrito, na representação de quasi-spin, em termos dos operadores escalonados $j_{i}$ como

$$
\frac{1}{2 J} H_{B H_{2}} \equiv h_{B H_{2}}=\frac{\mathcal{E}}{2} j_{3}-\eta j_{1}+\frac{\Lambda N}{4}\left[1+\frac{1}{N}+j_{3}^{2}\right],
$$

e se tratarmos os $j_{i}$ como variáveis clássicas, este Hamiltoniano pode ser usado para obter as equações de movimento através dos parênteses de Poisson (4.15), ou seja, utilizando

$$
\frac{d j_{i}}{d t}=\lim _{J \rightarrow \infty} \frac{d}{d t} \frac{J_{i}}{J}=\lim _{J \rightarrow \infty} \frac{2 J}{\mathrm{i}}\left[\frac{J_{i}}{J}, \frac{H_{B H_{2}}}{2 J}\right] \equiv 2\left\{j_{i}, h_{B H_{2}}^{(c l)}\right\}, \quad i=1,2,3 .
$$

onde $h_{B H_{2}}^{(c l)} \equiv \lim _{J \rightarrow \infty} h_{B H_{2}}$ denota a Hamiltoniana clássica. Um ponto a ser observado aqui é que a equação (4.17) introduz um escalonamento no tempo de tal forma que $t \rightarrow t / J$.

De (4.17) podemos verificar que o quasi-spin total $\sum_{i=1}^{3} j_{i}^{2}$ é uma constante do movimento (clássica) e consequentemente que sua dinâmica está restrita a uma esfera de raio unitário no espaço de quasi-spin. Fica claro então que as variáveis $j_{i}$ são de fato as componentes cartesianas de um vetor unitário de quasi-spin clássico, cuja dinâmica é dada pelas equações de movimento (4.17). Podemos então reformular a dinâmica do sistema em termos de coordenadas polares esféricas, ou seja, utilizando o ângulo polar $(0 \leq \theta \leq \pi)$ que o vetor de quasi-spin forma com o eixo $j_{3}$ positivo e o ângulo azimutal $(0 \leq \varphi \leq 2 \pi)$ no plano $j_{1} \times j_{2}$ que o vetor forma com o eixo $j_{1}$ positivo. Nestas coordenadas as componentes do vetor de quasi-spin ficam 


$$
\begin{aligned}
& j_{1}=\sin \theta \cos \varphi, \\
& j_{2}=\sin \theta \sin \varphi, \\
& j_{3}=\cos \theta .
\end{aligned}
$$

Com isso a Hamiltoniano "clássica", escrito em termos das variáveis canônicas $j_{3}$ e $\varphi$, no limite $J \rightarrow \infty$ fica

$$
h_{B H_{2}}^{(c l)}=\frac{\tilde{\Lambda}}{4}\left[1+j_{3}^{2}\right]+\frac{\mathcal{E}}{2} j_{3}-\eta \sqrt{1-j_{3}^{2}} \cos \varphi,
$$

onde utilizamos a relação $\sin \theta=\sqrt{1-j_{3}^{2}}$ e a definição $\Lambda N=\tilde{\Lambda}$ e as equações canônicas de movimento ficam então

$$
\begin{aligned}
\frac{d \varphi}{d t} & =2 \frac{\partial h_{B H_{2}}^{(c l)}}{\partial j_{3}}=\mathcal{E}+\tilde{\Lambda} j_{3}+2 \eta \frac{j_{3}}{\sqrt{1-j_{3}^{2}}} \cos \varphi \\
\frac{d j_{3}}{d t} & =-2 \frac{\partial h_{B H_{2}}^{(c l)}}{\partial \varphi}=2 \eta \sqrt{1-j_{3}^{2}} \sin \varphi,
\end{aligned}
$$

e os estados estacionários são os valores de $j_{3}$ e $\varphi$ para os quais estas equações se anulam. Isto implica em $\sin \varphi=0$, tal que $\varphi=0$ ou $\varphi=\pi$, onde os valores de $j_{3}$ correspondentes são soluções de

$$
j_{3}\left(\tilde{\Lambda}+2 \frac{\eta \cos \varphi}{\sqrt{1-j_{3}^{2}}}\right)=-\mathcal{E}, \quad \cos \varphi= \pm 1
$$

A figura 4.7 mostra as curvas de nível de (4.18), em termos de $\varphi$ e $j_{3}$, para $\tilde{\Lambda}=-2$ e $\eta=\mathcal{E}=0.2$. Podemos notar a existência de uma separação do movimento em órbitas com características dinâmicas diferentes: por exemplo, as curvas $h_{\mathrm{BH}_{2}}^{(c l)}=-1$ e $h_{\mathrm{BH}_{2}}^{(c l)}=-1.1$ correspondem a órbitas periódicas, que quânticamente estão associadas ao regime de self-trapping, ou seja, à uma localização preferencial das das partículas em um dos dois modos localizados; enquanto que as curvas a partir de $h_{\mathrm{BH}_{2}}^{(\mathrm{cl})}=-1.2$ correspondem à órbitas não limitadas que quanticamente estão associadas ao regime de oscilações Josephson, ou seja, não existe localização preferencial das partículas em apenas um dos dois modos localizados e os átomos possuem energia suficiente para se mover de um modo localizado para o outro. Esta separação de regimes também pode ser vista na figura 4.1 para diferentes valores dos parâmetros dos Hamiltoniano quântico. Nas figuras 4.3 e 4.4 podemos ver como o espalhamento do pacote inicial se altera conforme escolhemos uma condição inicial na região correspondente respectivamente ao regime de self-trapping e à separatriz do movimento. Vemos claramente que o pacote inicial se espalha consideravelmente mais rápido neste último caso. 
Note a semelhança entre a figura 4.7 e a figura 4.2(b). Esta semelhança torna evidente a correspondência entre $h_{B H_{2}}$ e $h_{B H_{2}}^{(c l)}$ para um valor de $N$ suficientemente grande $(N=64$ na figura 4.2(b)), o que demonstra a importância do escalonamento de $H_{B H_{2}}$ em (4.16) ao considerarmos o limite $N \rightarrow \infty$ como limite clássico.

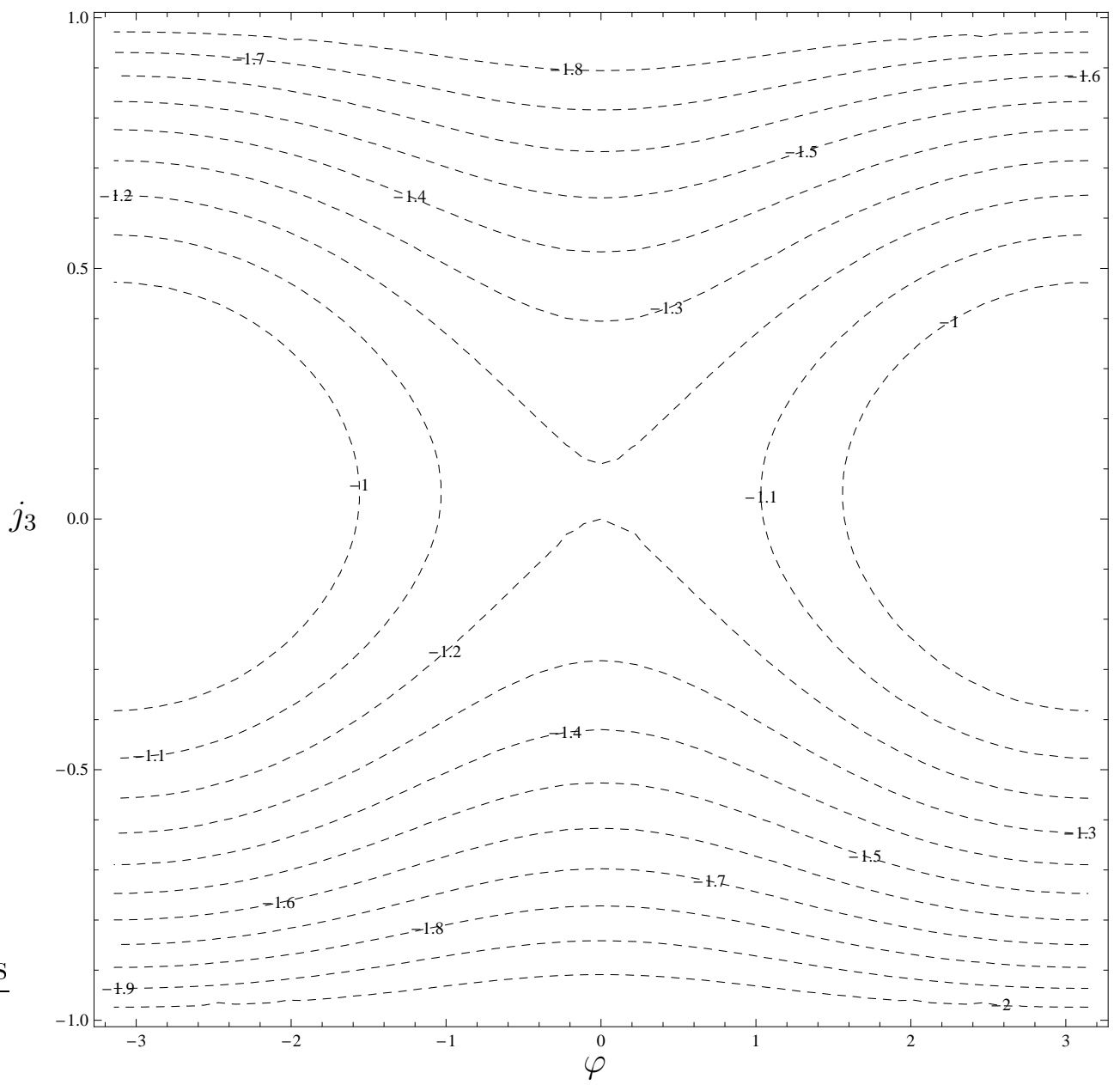

Figura 4.7: Curvas de nível da Hamiltoniana clássica (4.18) para $\tilde{\Lambda}=-2$ e $\eta=\mathcal{E}=0.2$ (cf. figura $4.2)$.

\subsection{Heterogeneidade Distribucional no Modelo de Bose-Hubbard}

\subsubsection{Estado Inicial e Cálculo de $\tilde{h}_{q}(\hat{\rho})$}

Nesta seção descreveremos alguns dos resultados obtidos no estudo da heterogeneidade distribucional no espaço de fases $\tilde{h}_{q}(\hat{\rho})$ para o modelo de Bose-Hubbard com dois sítios e $N$ bósons. É possível adiantar que, como já era esperado, o comportamento de $\tilde{h}_{q}(\hat{\rho})$ é fortemente dependente, pelo menos quantitativamente, do estado inicial, ou seja, da posição da distribuição inicial no 
espaço de fases e da forma da distribuição inicial. Este fato pode ser verificado nas figuras 4.3 a 4.5 .

Consideremos então inicialmente, ao invés do pacote inicial coerente (4.9) uma variação deste dada por

$$
\left|\Psi_{0}^{\mathrm{coh}}\right\rangle \equiv|\xi, \vartheta\rangle_{N}+|\xi,-\vartheta\rangle_{N}
$$

onde $|\xi, \vartheta\rangle_{N}$ é dado por (4.11), ou seja, ao invés de apenas um pico localizado ao redor de um ponto do espaço de fases teremos uma superposição coerente de dois picos. A representação de Wigner no espaço de fases discreto do estado (4.20) é mostrada na figura 4.8.

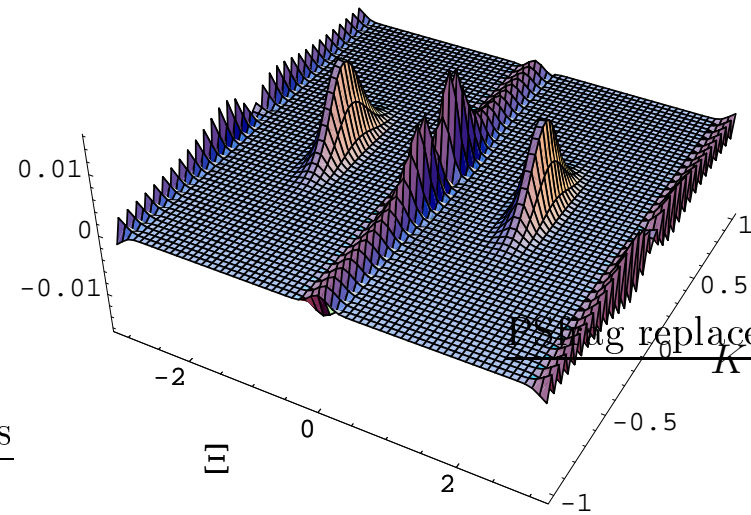

(a)

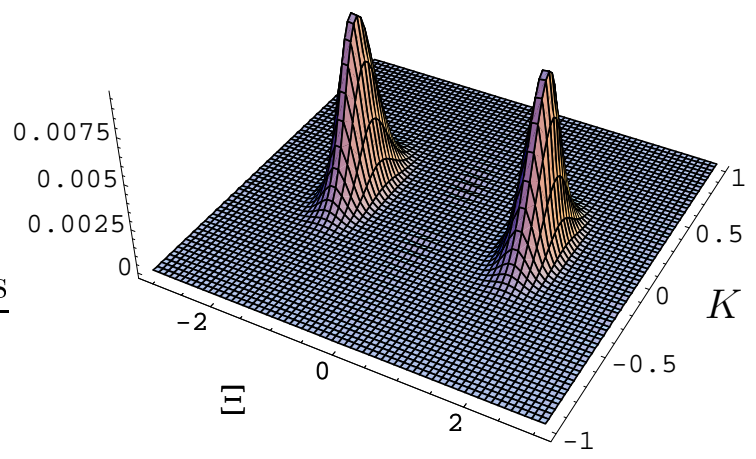

(b)

Figura 4.8: Funções de Wigner discreta de $\hat{\rho}$ (esquerda) e $\hat{\rho}_{\alpha}$ (direita), para o estado (4.20) em $t=0$. Os valores dos parâmetros são $\Lambda=-2, \eta=\mathcal{E}=0.2, N=100, M=7, K_{0}=0, \Xi_{0}=1.5 \mathrm{e}$ $\alpha=4$.

Deve-se notar na figura 4.8, além dos dois picos principais, a existência de um padrão oscilatório ao longo da região $K=0$, que aparece devido à superposição coerente entre os dois picos. Perceba que, diferentemente do caso (4.9), neste caso o estado inicial possui uma estrutura mais complexa já em $t=0$, inclusive com valores negativos de $\rho^{W}(\Xi, K)$, o que para (4.9) ocorria apenas para $t \neq 0$. De acordo com $[53,54,55]$ este padrão oscilatório (interferências) bem como os valores negativos de $\rho^{W}(\Xi, K)$, estão diretamente relacionados à correlações quânticas do sistema e este se torna "mais clássico" à medida que estas oscilções são amortecidas, ou seja, este padrão oscilatório pode ser visto como sendo uma espécie de "assinatura quântica" do sistema.

O papel da média (coarse-graining) neste caso, é principalmente o de amortecer (alisar) este padrão oscilatório e em particular os valores negativos da função de Wigner. Este efeito pode ser observado diretamente na figura 4.8, onde também é possível observar que a altura do dois picos principais também é afetada pela média, mas com uma intensidade bem menor que as oscilações, que praticamente desaparecem, pelo menos para a média utilizada.

Um fato interessante que verificamos ao estudar o efeito da média (coarse-graining) no espaço de 
fases, foi que ao olharmos para os autovetores da matriz densidade correspondente ao operador $\hat{\rho}_{\alpha}$ notamos que o padrão oscilatório (interferências) existente em $\hat{\rho}$ e que é aparentemente suprimido em $\hat{\rho}_{\alpha}$, na verdade foi "transferido" para os autovetores de $\hat{\rho}_{\alpha}$. Este fato fica claro nas figuras 4.9 a 4.11 .

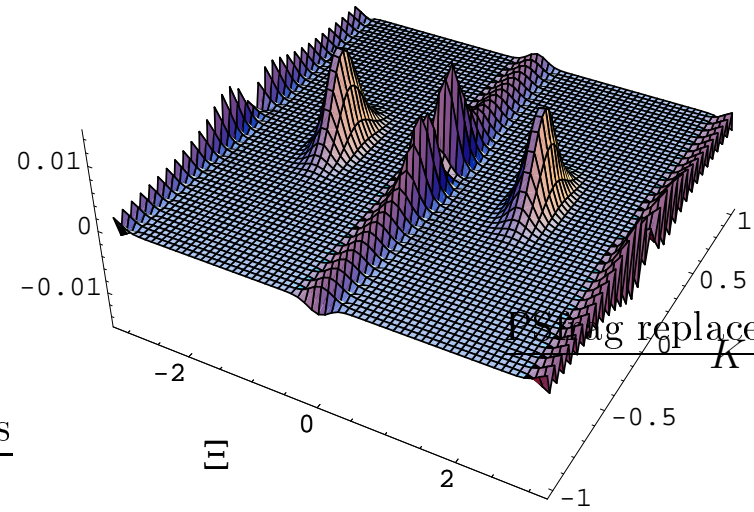

(a)

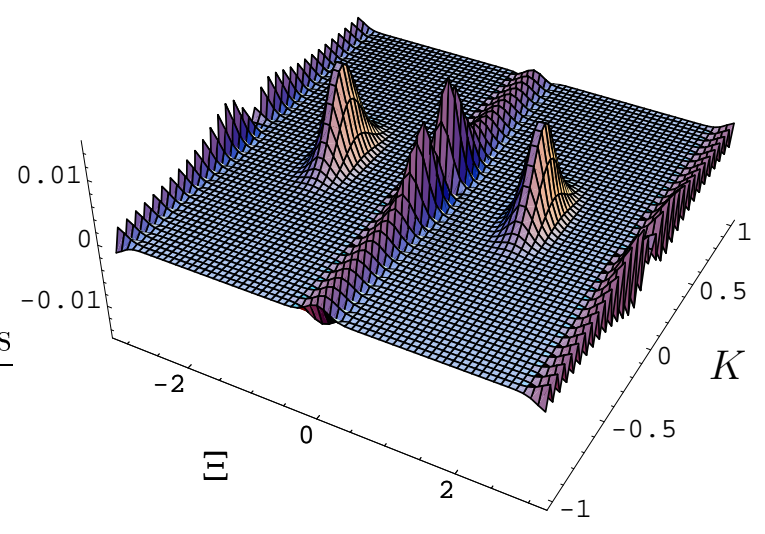

(b)

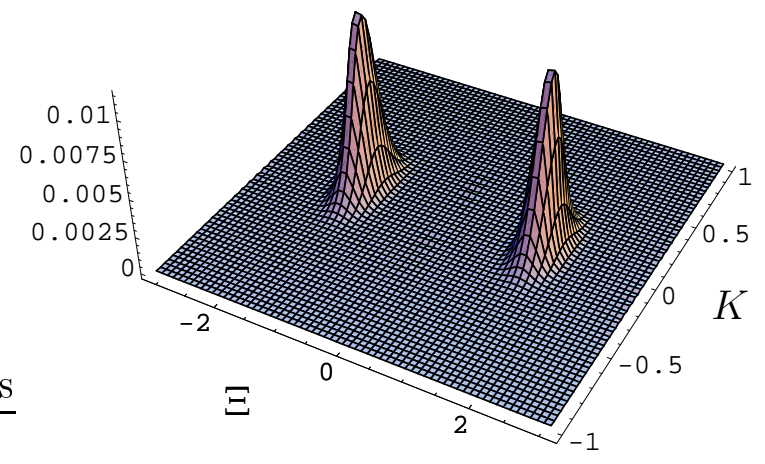

(c)

Figura 4.9: Nos gráficos do topo temos as funções de Wigner $\rho_{\alpha, 1}^{W}$ e $\rho_{\alpha, 2}^{W}$ dos autovetores correpondentes aos dois maiores autovalores da matriz de $\hat{\rho}_{\alpha}\left(\lambda_{1} \simeq 0.396013\right.$ (a) e $\lambda_{2} \simeq 0.391274$ (b)), para o estado inicial (4.20) em $t=0$. No gráfico de baixo, temos a soma ponderada $\lambda_{1} \rho_{\alpha, 1}^{W}+\lambda_{2} \rho_{\alpha, 2}^{W}$. 


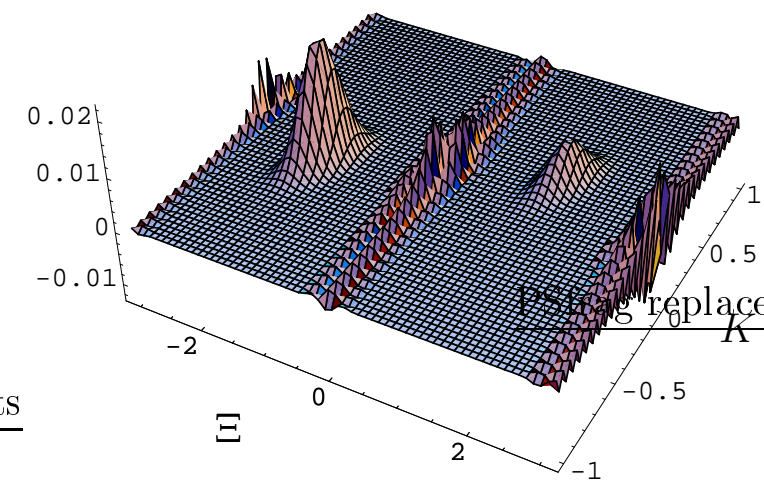

(a)

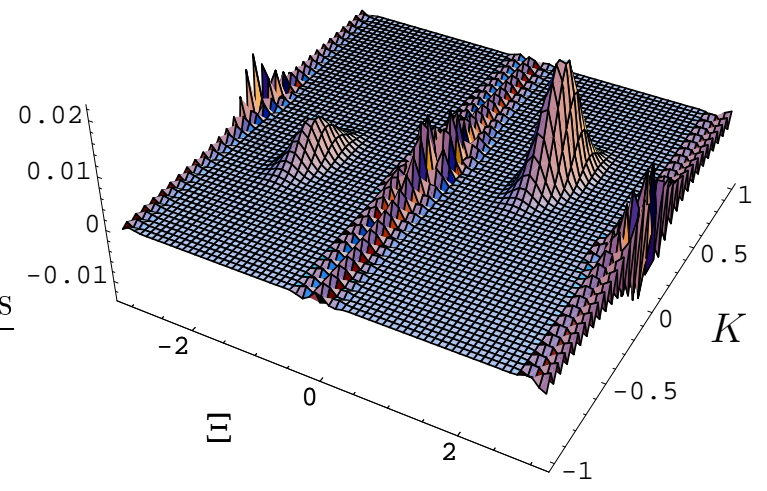

(b)

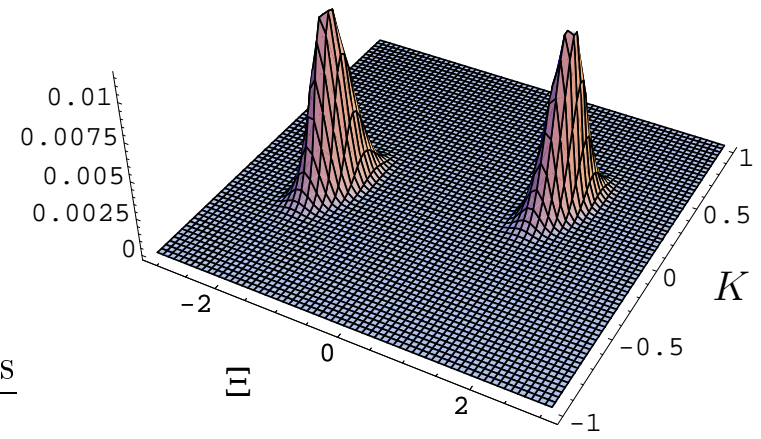

(c)

Figura 4.10: O mesmo da figura 4.9 para $t=15$. Neste caso, $\lambda_{1} \simeq 0.39122$ (a) e $\lambda_{2} \simeq 0.38898(\mathrm{~b})$. 


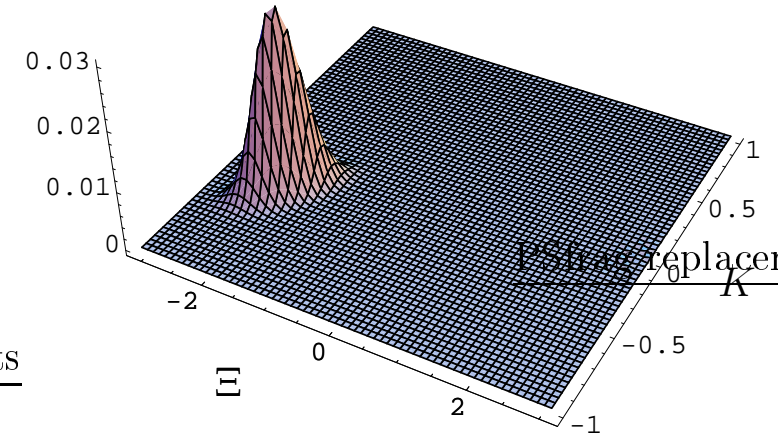

(a)

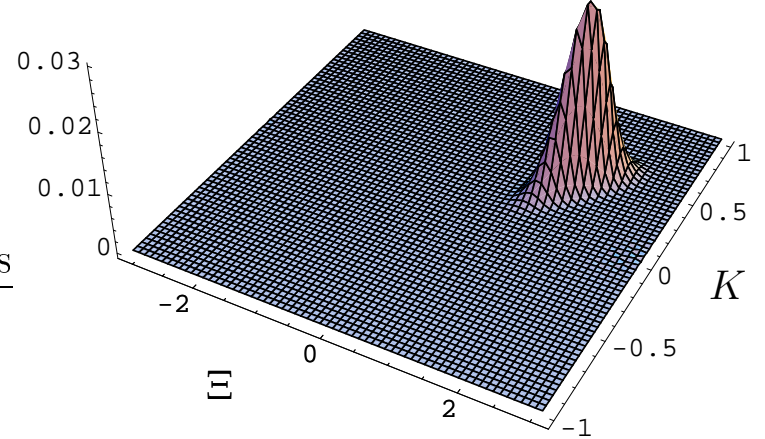

(b)

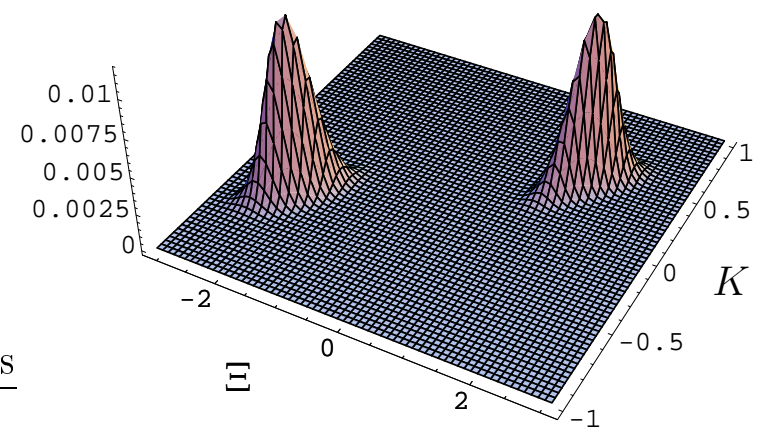

(c)

Figura 4.11: O mesmo da figura 4.9 para $t=30$. Neste caso, $\lambda_{1} \simeq 0.38612$ (a) e $\lambda_{2} \simeq 0.37882(\mathrm{~b})$. 
Vemos claramente nas figuras 4.9 e 4.10 que apesar do estado $\hat{\rho}_{\alpha}$ ser localizado no espaço de fases, os autovetores da matriz correspondente são extremamente deslocalizados. Isto é possível devido à "complementaridade" dos autovetores (que fica bem evidente na figura 4.10), pois estes ao serem combinados provocam o cancelamento das interferências no estado total $\hat{\rho}_{\alpha}$. Este comportamento se modifica à medida que o sistema unitariamente evolui no tempo. Esta mudança fica evidente na figura 4.11, onde os autovetores dominantes são extremamente localizados. Podemos ver também, para os três instantes de tempo, que os dois autovetores dominantes contém praticamente toda a informação sobre o estado, pelo menos aquela necessária à construção da função de Wigner de $\hat{\rho}_{\alpha}$.

Estas observações nos dão uma idéia mais clara de como a média (coarse-graining) atua no espaço de fases, mais especificamente como esta "introduz" decoerência no sistema ${ }^{3}$. Consequentemente, pelo menos neste caso, é preciso ser cuidadoso ao afirmar que a eliminação das interferências no espaço de fases é condição suficiente para que o sistema se torne clássico, como afirmado em $[53,54,55]$.

Por fim, a figura 4.12 mostra a evolução temporal de $\tilde{h}_{q}(\hat{\rho})$, para o estado inicial (4.20) e uma média do tipo (3.47), para diferentes valores de $N$. Como mencionado anteriormente (ver discussão ao final do capítulo 3 , logo após a equação (3.50)) o fato de utilizarmos a definição de $\tilde{h}_{q}(\hat{\rho})(3.46)$ em termos da variancia $v(\alpha)$ permite que nos aproximemos mais do valor $\alpha=0$ do que a definição de $h_{q}(\hat{\rho})$ (2.45) em termos de $\alpha$ apenas. No caso da figura 4.12, foi possível utilizar um valor $\alpha=0.04$, sendo que para valores de $\alpha$ abaixo deste, devido as limitações de precisão do cálculo numérico, $\tilde{h}_{q}(\hat{\rho})$ se comporta de forma bastante instável e em seguida se torna identicamente nula, como pode ser visto na figura 4.13.

\footnotetext{
${ }^{3}$ Eliminação do padrão oscilatório (interferências).
} 

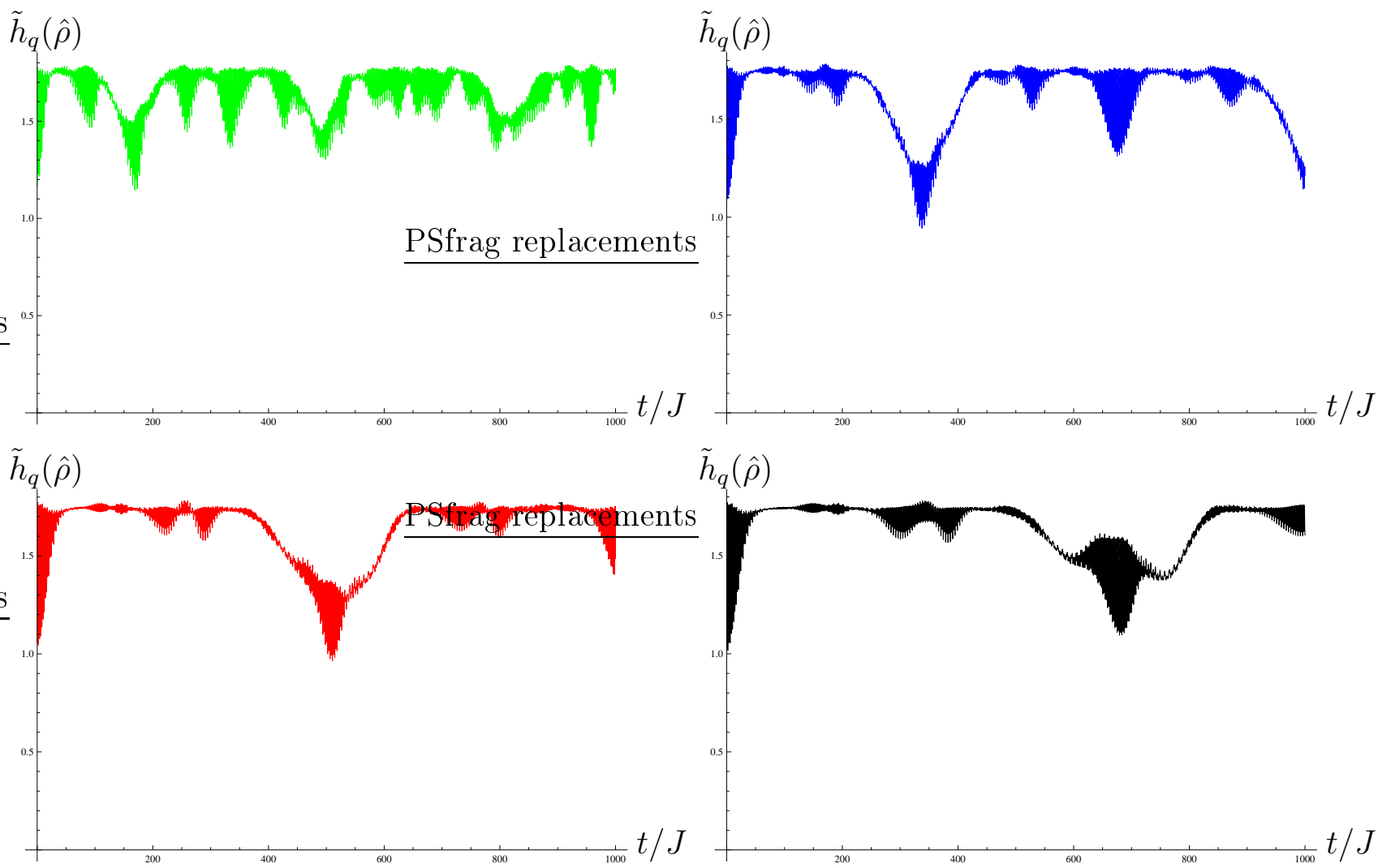

$\tilde{h}_{q}(\hat{\rho})$

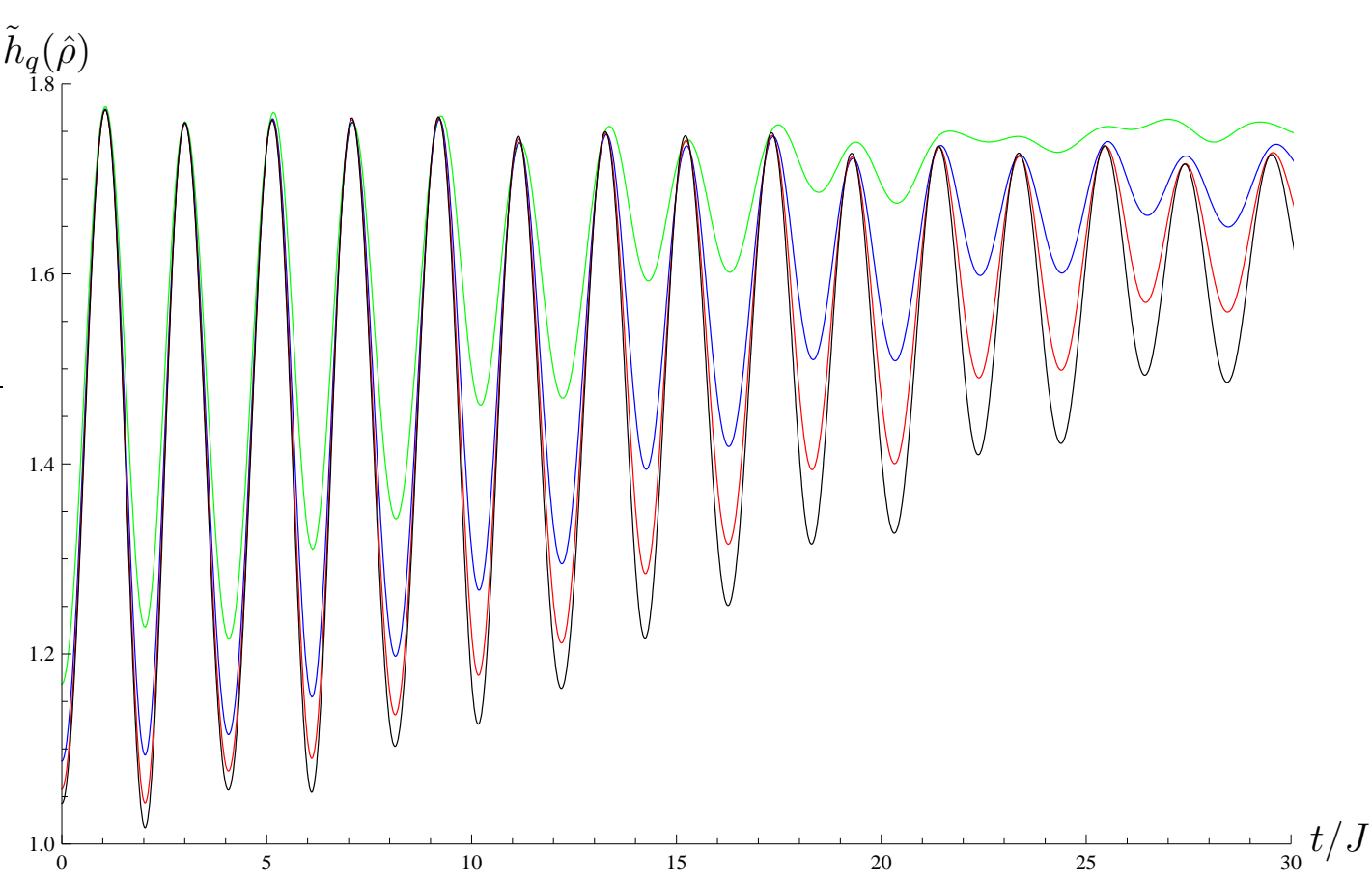

Figura 4.12: Comportamento da heterogeneidade distribucional $\tilde{h}_{q}(\hat{\rho})$ para o estado inicial (4.20) como função do tempo escalonado $t / J$. Os valores dos parâmetros são os mesmos da figura 4.8, exceto por $\alpha=0.04$. As diferentes cores referem-se aos diferentes valores de $N=50$ (verde), 100 (azul), 150 (vermelho) e 200 (preto). 


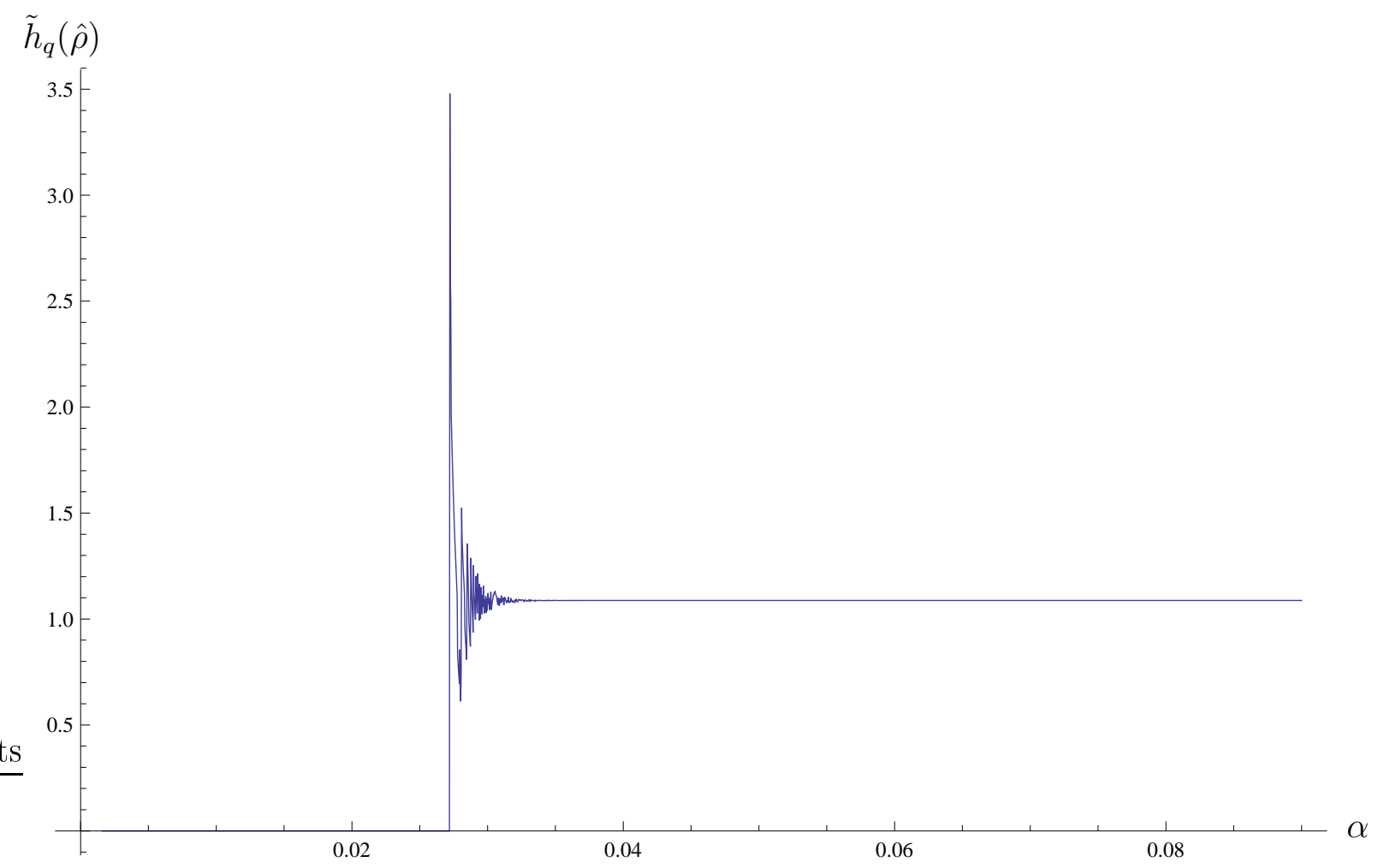

Figura 4.13: Heterogeneidade distribucional $\tilde{h}_{q}(\hat{\rho})$ como função do parâmetro da média (coarsegraining) $\alpha$ para $N=100$ e $t / J=0$. Os valores dos demais parâmetros e o estado inicial são os mesmos da figura 4.12 .

O primeiro ponto importante que podemos observar na figura 4.12 é que analisando estes comportamentos para tempos mais longos $(t / J \sim 1000)$, verificamos a existência de um comportamento (quase) periódico, cujo período muda com o valor de $N$. Além disso, podemos ver que existem diversos "sub-períodos" que também mudam com o valor de $N$. Acreditamos que a existência destes "sub-períodos" esteja relacionada com a distribuição dos autovalores de energia. Uma observação que corrobora esta suposição, como veremos no capítulo 5, é o fato desta "sub-periodicidade" não ocorrer, ou pelo menos ser menos evidente, no caso de um sistema classicamente caótico. Outro fato importante que devemos mencionar é que o comportamento periódico exibido por $\tilde{h}_{q}(\hat{\rho})$ está de pleno acordo com os resultados obtidos na literatura, para sistemas classicamente integráveis (ver discussão ao final do capítulo 2, em especial o penúltimo parágrafo). Este fato é mais uma evidência da sensibilidade de $\tilde{h}_{q}(\hat{\rho})$ para medir a variação da estrutura da distribuição no espaço de fases (neste caso, discreto).

\subsubsection{Correspondência Quântico-Clássica}

Do ponto de vista da correspondência quântico-clássica, faz mais sentido comparar o comportamento da heterogeneidade distribucional clássica $h_{c}(\rho)$ com a quântica $\tilde{h}_{q}(\hat{\rho})$ para um estado inicial "liso", ao invés de um estado que possua estruturas de interferência como é o estado (4.20). 
Esta abordagem nos permite ver mais claramente a diferença entre os casos clássico e quântico, pois classicamente a distribuição pode adquirir estruturas em escalas arbitrariamente pequenas, o que não ocorre no caso quântico devido ao princípio da incerteza.

Consideremos então, no lugar da superposição coerente dos estados $|\xi, \vartheta\rangle_{N}$ e $|\xi,-\vartheta\rangle_{N}$ como em (4.20), um estado inicial que é uma superposição "incoerente" destes estados. Este estado pode ser representado por

$$
\hat{\rho}_{0}^{\text {incoh }} \equiv \frac{1}{2}\left(\hat{\rho}_{+}+\hat{\rho}_{-}\right)
$$

onde

$$
\hat{\rho}_{ \pm} \equiv|\xi, \pm \vartheta\rangle_{N N}\langle\xi, \pm \vartheta|
$$

A principal diferença entre os estados (4.20) e (4.21) é a ausência de franjas de interferência no segundo. A figura 4.14 nos mostra o comportamento de $\tilde{h}_{q}(\hat{\rho})$ para o estado $(4.21)$, onde podemos ver que o comportamento (quase) periódico se mantém.
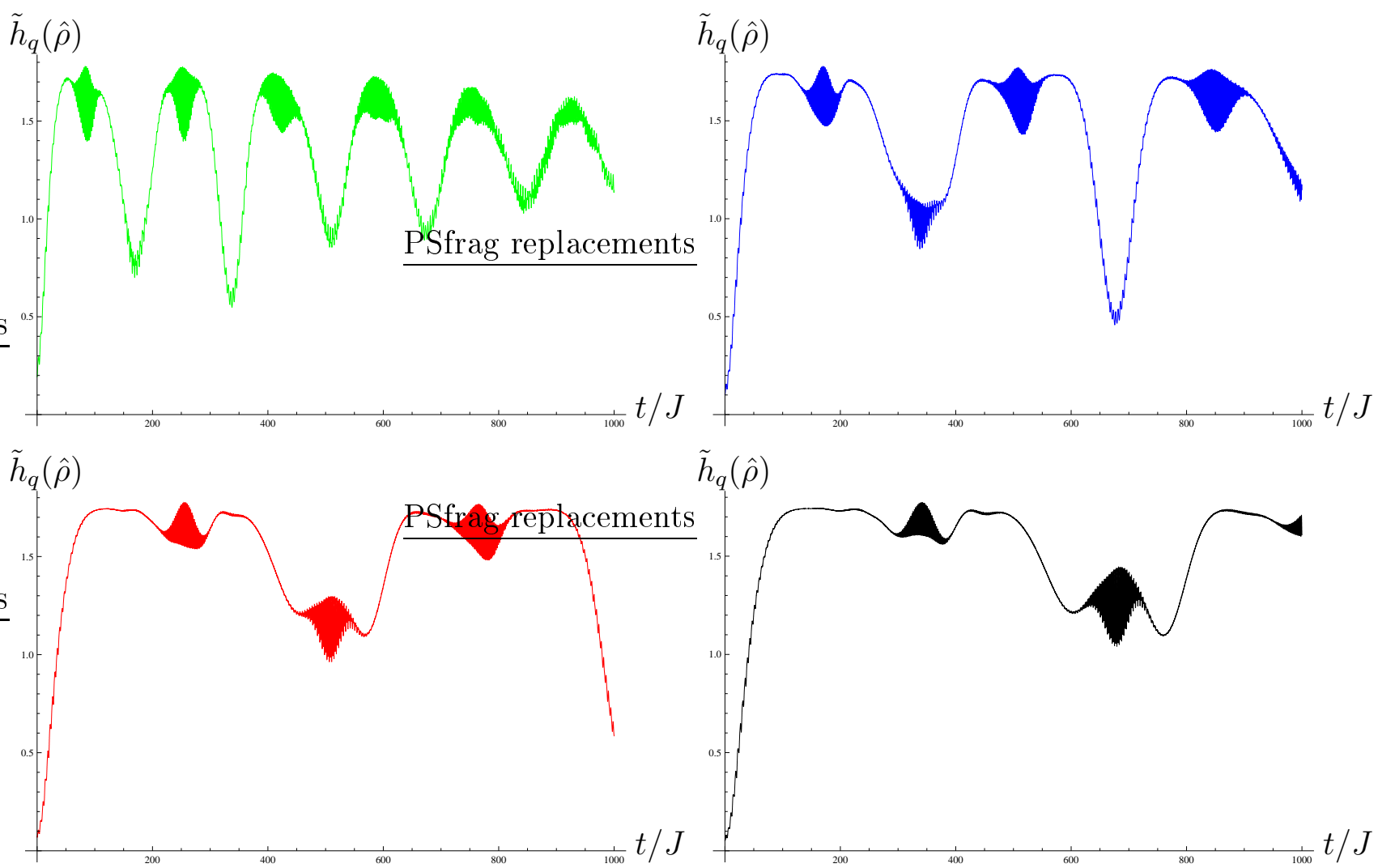

Figura 4.14: Comportamento de $\tilde{h}_{q}(\hat{\rho})$ para o estado inicial $\hat{\rho}_{0}^{\text {incoh }}$ em $(4.21)$. Os valores dos parâmetros e a descrição das cores são os mesmos da figura 4.12.

Um ponto interessante, sobre o qual podemos nos questionar, é a respeito da influência que a 
existência de um padrão oscilatório (interferências) no estado inicial tem sobre o comportamento de $\tilde{h}_{q}(\hat{\rho})$. A figura 4.15 nos dá uma idéia dessa influência ${ }^{4}$. Nela vemos que em $t=0$ existe uma separação nítida entre o caso coerente e o caso "incoerente", o que já era esperado pois a função de Wigner no caso coerente apresenta estruturas oscilatórias (interferências) provenientes de correlações entre os picos, enquanto que no caso "incoerente" a função de Wigner é completamente lisa no espaço de fases. Logo podemos concluir que um maior (menor) valor de $\tilde{h}_{q}(\hat{\rho})$ indica uma maior (menor) deslocalização da distribuição (neste caso da função de Wigner) no espaço de fases, e vice-versa. Esta propriedade de $\tilde{h}_{q}(\hat{\rho})$ já havia sido observada no caso de um espaço de dimensão infinita em [24].

Outra observação que podemos fazer sobre a figura 4.15, é que em ambos os casos (coerente e "incoerente") $\tilde{h}_{q}(\hat{\rho})$ exibe um comportamento (quase) periódico, o que, novamente enfatizando, está de acordo com os resultados na literatura, e.g. [16, 18], para o caso de sistemas classicamente integráveis. Note que qualitativamente os comportamentos são muito parecidos, mudando apenas a amplitude de oscilação, o que novamente indica o efeito quantitativo que as interferências existentes em $\hat{\rho}$ exercem sobre o comportamento de $\tilde{h}_{q}(\hat{\rho})$.

\footnotetext{
${ }^{4}$ Omitimos aqui o caso (4.9), pois estamos interessados principalmente no efeito das correlações quânticas sobre o comportamento de $\tilde{h}_{q}(\hat{\rho})$
} 

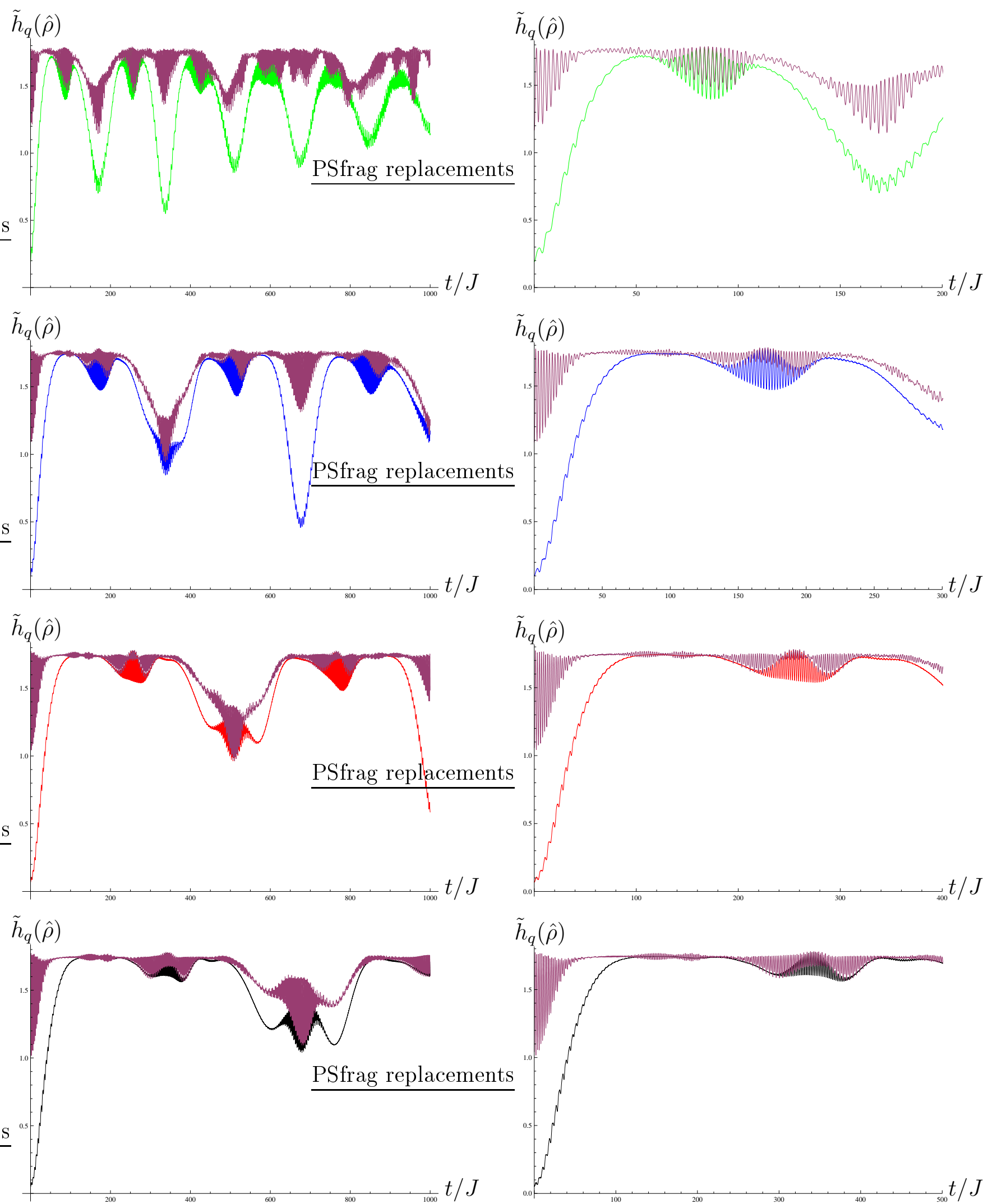

Figura 4.15: Comportamento de $\tilde{h}_{q}(\hat{\rho})$ com $t / J$ para os estados $(4.21)$ e $(4.20)$. Os valores dos parâmetros e a descrição das cores são os mesmos das figuras 4.12 e 4.14, exceto pelas curvas magenta que neste caso representam $\tilde{h}_{q}(\hat{\rho})$ para o estado inicial (4.20), com o respectivo valor de $N$. 
A figura 4.16 mostra o comportamento da heterogeneidade quântica $\tilde{h}_{q}(\hat{\rho})$ (3.46) e clássica $h_{c}(\rho)$ (2.41) com o tempo $t$ ( $t / J$ no caso quântico), para o estado (4.21) e diferentes valores de $N$ no caso quântico. A distribuição inicial clássica foi obtida, neste caso, solucionando as equações de movimento (4.19) e reconstruindo a distribuição inicial através de uma evolução temporal inversa, de tal forma que em $t=0$ a distribuição possua a forma desejada, neste caso a mesma forma da função de Wigner do estado $\hat{\rho}_{0}^{\text {incoh }}$ (4.21), que é exatamente a forma da figura (4.8)(b). Como a construção da distribuição inicial clássica $\rho$ requer uma discretização do intervalo de definição das variáveis clássicas $j_{3} \in[-1,1]$ e $\varphi \in[-\pi, \pi]$, a heterogeneidade distribucional clássica $h_{c}(\rho)$ é calculada através das definições (2.39), (2.40) e (2.41)(1), exceto pelo fato de que em (2.39) a integral usual é substituída pela soma de Riemann

$$
\sum_{k=1}^{n}\left|\Delta j_{3}^{(k)} \Delta \varphi^{(k)} \rho\left(x^{(k)}, y^{(k)}\right)\right|^{2}
$$

onde $n$ é o número de partições dos domínios de $j_{3}$ e $\varphi\left(n=200\right.$ na figura 4.16), $\Delta j_{3}^{(k)}=j_{3}^{(k)}-j_{3}^{(k-1)}$ e $\Delta \varphi^{(k)}=\varphi^{(k)}-\varphi^{(k-1)}$ são os tamanhos destas partições e $j_{3}^{(k-1)} \leq x^{(k)} \leq j_{3}^{(k)}$ e $\varphi^{(k-1)} \leq y^{(k)} \leq \varphi^{(k)}$. Na figura 4.16 consideramos $n=200$ partições iguais, ou seja, $\Delta j_{3}^{(k)}=\Delta j_{3}=2 / n$ e $\Delta \varphi^{(k)}=\Delta \varphi=$ $2 \pi / n$.

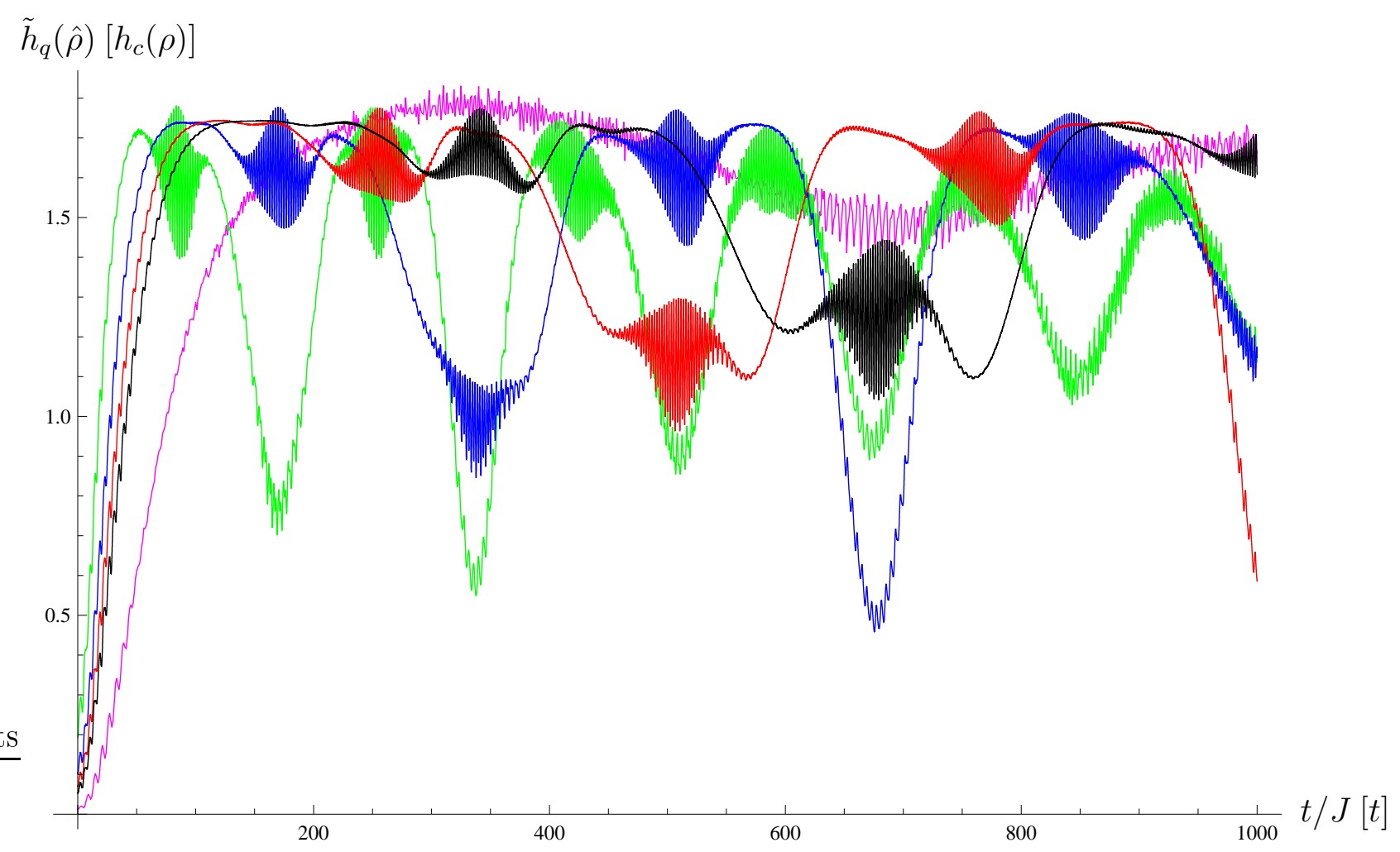

Figura 4.16: Comportamento de $\tilde{h}_{q}(\hat{\rho})$ e $h_{c}(\rho)$ para um estado inicial da forma (4.21). Os valores dos parâmetros e a descrição das cores são os mesmos da figura 4.15, exceto pela cor magenta que neste caso representa a heterogeneidade clássica $h_{c}(\rho)$. 
Para $t(t / J)=0$, vemos que a heterogeneidade distribucional possui praticamente o mesmo valor para todos os casos (quânticos e clássico). Isto se dá devido ao fato do estado quântico "incoerente" ser um estado liso inicialmente, ou seja, sem oscilações e consequentemente mais próximo de um estado clássico. Para $t(t / J)>0$, temos que inicialmente a distribuição adquire estrura mais rapidamente no caso quântico $\tilde{h}_{q}(\hat{\rho})$ (independendo do valor de $N$ ) do que no caso clássico $h_{c}(\rho)$, o que também já era esperado devido ao fato da função de Wigner $\rho^{W}$ seguir a mesma evolução temporal da distribuição clássica $\rho$ no espaço de fases, mas com a presença de interferências [18]. No entanto, vemos que este crescimenteo mais rápido de $\tilde{h}_{q}(\hat{\rho})$ não se mantém para $t(t / J) \gtrsim 200$. Isto se deve ao fato de que a função de Wigner resiste ao aparecimento de estruturas em escalas muito pequenas no espaço de fases devido ao princípio de incerteza, o que não acontece no caso da distribuição clássica.

Outro ponto interessante é o fato de que o tempo necessário para que apareçam as maiores recorrências (oscilações de maior amplitude) no valor de $\tilde{h}_{q}(\hat{\rho})$, ou seja, para que a forma do estado evoluido se aproxime da forma do estado inicial, aumenta conforme $N$ aumenta. Esta ocorrência está essencialmente relacionada à dimensionalidade finita do espaço de Hilbert do sistema (neste caso temos $N+1$ estados), devido a qual a distribuição (função de Wigner), não podendo espalharse indefinidamente, leva um certo tempo, que depende de $N$, para "se aproximar" do estado inicial.

Do ponto de vista da correspondência quântico-clássica, a figura 4.16 nos mostra que, pelo menos em termos da quantidade de estrutura da distribuição (função de Wigner) no espaço de fases, o comportamento inicial da função de Wigner $\rho^{W}$ se aproxima do comportamento inicial da distribuição clássica $\rho$ à medida que $N$ aumenta e que aparentemente é possível obter uma correspondência quântico-clássica tão "boa" quanto se queira, bastando para isso, neste caso, considerar um valor de $N$ suficientemente grande. Esta observação está de acordo com o fato de que o limite $N \rightarrow \infty$ corresponde de certa forma ao limite clássico do sistema, o que já é um resultado bastante satisfatório.

Tendo estudado o comportamento de $\tilde{h}_{q}(\hat{\rho})$ e verificado que, para sistemas classicamente integráveis, este comportamento está de acordo com os resultados obtidos na literatura, podemos enfim passar para o estudo de sistemas cujo análogo clássico apresenta um comportamento caótico. Isto será feito no capítulo 5 para um modelo com dois graus de liberdade. 


\section{Capítulo 5}

\section{Modelo com Dois Graus de Liberdade}

Neste capítulo daremos continuidade ao estudo do comportamento da heterogeneidade distribuci-

onal no espaço de fases $\tilde{h}_{q}(\hat{\rho})$. Com o intuito de fazê-lo, agora para sistemas cujo análogo clássico apresenta um comportamento caótico, consideraremos um modelo que apresenta uma propensão coletiva semelhante à observada no modelo de Bose-Hubbard com dois sítios e $N$ bósons. Este sistema, após uma escolha conveniente dos parâmetros, se reduz ao sistema de dois momentos angulares acoplados estudado em [56], cujo comportamento clássico correspondente pode apresentar caos a partir de um valor crítico do parâmetro de acoplamento. O sistema completo, foi estudado em [57] e também apresenta características típicas de um sistema classicamente caótico (e.g., cruzamentos evitados de níveis de energia). No entanto, o sistema estudado por Hines, além de também apresentar as propriedades pertinentes ao nosso estudo neste trabalho (propensão coletiva e comportamento clássico bem definido), tem a vantagem de possuir uma descrição mais simples e portanto mais fácil de ser tratada.

\subsection{Hamiltoniano do Sistema, Representação de Quasi-Spin e Limite Termodinâmico}

Um modelo com propensão coletiva bastante interessante, que pode apresentar um comportamento mais complexo do que o modelo de Bose-Hubbard com dois sítios e $N$ bósons e que além disso pode ser estudado utilizando a mesma abordagem é o sistema modelado pela densidade hamiltoniana

$$
\begin{aligned}
\mathcal{H}= & \frac{\lambda_{a}^{(1)}}{2 V^{2}} a_{1}^{\dagger} a_{1}^{\dagger} a_{1} a_{1}+\frac{\lambda_{a}^{(2)}}{2 V^{2}} a_{2}^{\dagger} a_{2}^{\dagger} a_{2} a_{2}+\frac{\mathcal{E}_{a}^{(1)}}{V} a_{1}^{\dagger} a_{1}+\frac{\mathcal{E}_{a}^{(2)}}{V} a_{2}^{\dagger} a_{2}+\frac{\eta_{a}}{V}\left[a_{1}^{\dagger} a_{2}+a_{2}^{\dagger} a_{1}\right]+ \\
& +\frac{\lambda_{b}^{(1)}}{2 V^{2}} b_{1}^{\dagger} b_{1}^{\dagger} b_{1} b_{1}+\frac{\lambda_{b}^{(2)}}{2 V^{2}} b_{2}^{\dagger} b_{2}^{\dagger} b_{2} b_{2}+\frac{\mathcal{E}_{b}^{(1)}}{V} b_{1}^{\dagger} b_{1}+\frac{\mathcal{E}_{b}^{(2)}}{V} b_{2}^{\dagger} b_{2}+\frac{\eta_{b}}{V}\left[b_{1}^{\dagger} b_{2}+b_{2}^{\dagger} b_{1}\right]+ \\
& +\frac{\lambda_{a b}^{(1)}}{V^{2}} a_{1}^{\dagger} b_{1}^{\dagger} b_{1} a_{1}+\frac{\lambda_{a b}^{(2)}}{V^{2}} a_{2}^{\dagger} b_{2}^{\dagger} b_{2} a_{2} .
\end{aligned}
$$


Este sistema pode ser usado para descrever, por exemplo, um condensado de Bose-Einstein de duas componentes $a$ e $b$, submetido a uma armadilha óptica do tipo poço duplo (índices 1 e 2). Como veremos mais adiante, o análogo clássico de (5.1) é um sistema de dois graus de liberdade e portanto possui uma dinâmica mais complexa do que o modelo de Bose-Hubbard com dois sítios e $N$ bósons, podendo até apresentar um comportamento caótico. A dinâmica deste sistema foi estudada nos trabalhos de $\mathrm{Li}$ [57] e $\mathrm{Ng}$ [58, 59], nos quais foram observados comportamentos interessantes. No primeiro, foi verificada a existência de cruzamentos evitados dos níveis de energia, o que pode ser um indício de comportamento caótico do sistema, além disso, foi demonstrada neste modelo a existência de auto armadilhamento. No segundo, foram investigadas propriedades de tunelamento entre os modos localizados e foi observada a existência de emaranhamento quântico entre os modos. Contudo, o resultado que mais chama a atenção neste trabalho é a verificação de que átomos de duas espécies distintas, inicialmente localizadas em diferentes modos, podem tunelar sob a forma de pares emaranhados quando a interação entre espécies é suficientemente forte. Estes efeitos nos dão uma idéia de quão rico é o comportamente do modelo (5.1).

Consideremos então $\mathcal{H}=\mathcal{H}_{a}+\mathcal{H}_{b}+\mathcal{H}_{a b}$, onde

$$
\begin{gathered}
\mathcal{H}_{a} \rightarrow \frac{\lambda_{a}^{(1)}}{2 V^{2}} a_{1}^{\dagger} a_{1}^{\dagger} a_{1} a_{1}+\frac{\lambda_{a}^{(2)}}{2 V^{2}} a_{2}^{\dagger} a_{2}^{\dagger} a_{2} a_{2}+\frac{\mathcal{E}_{a}^{(1)}}{V} a_{1}^{\dagger} a_{1}+\frac{\mathcal{E}_{a}^{(2)}}{V} a_{2}^{\dagger} a_{2}+\frac{\eta_{a}}{V}\left[a_{1}^{\dagger} a_{2}+a_{2}^{\dagger} a_{1}\right], \\
\mathcal{H}_{b} \rightarrow \frac{\lambda_{b}^{(1)}}{2 V^{2}} b_{1}^{\dagger} b_{1}^{\dagger} b_{1} b_{1}+\frac{\lambda_{b}^{(2)}}{2 V^{2}} b_{2}^{\dagger} b_{2}^{\dagger} b_{2} b_{2}+\frac{\mathcal{E}_{b}^{(1)}}{V} b_{1}^{\dagger} b_{1}+\frac{\mathcal{E}_{b}^{(2)}}{V} b_{2}^{\dagger} b_{2}+\frac{\eta_{b}}{V}\left[b_{1}^{\dagger} b_{2}+b_{2}^{\dagger} b_{1}\right]
\end{gathered}
$$

e

$$
\mathcal{H}_{a b} \rightarrow \frac{\lambda_{a b}^{(1)}}{V^{2}} a_{1}^{\dagger} b_{1}^{\dagger} b_{1} a_{1}+\frac{\lambda_{a b}^{(2)}}{V^{2}} a_{2}^{\dagger} b_{2}^{\dagger} b_{2} a_{2}
$$

Note que neste caso o número de átomos de cada espécie $\left(N_{a}=a_{1}^{\dagger} a_{1}+a_{2}^{\dagger} a_{2}\right.$ e $\left.N_{b}=b_{1}^{\dagger} b_{1}+b_{2}^{\dagger} b_{2}\right)$ e o número total de átomos $\left(N=N_{a}+N_{b}\right)$ se conservam, ao contrário do número de átomos em cada poço $\left(N_{1}=a_{1}^{\dagger} a_{1}+b_{1}^{\dagger} b_{1}\right.$ e $\left.N_{2}=a_{2}^{\dagger} a_{2}+b_{2}^{\dagger} b_{2}\right)$. Utilizando agora a representação de Schwinger dos operadores de momento angular, definimos mais uma vez os operadores coletivos (quasi-spin)

$$
J_{ \pm}^{(a)}=J_{x}^{(a)} \pm J_{y}^{(a)} \equiv a_{1,2}^{\dagger} a_{2,1}, \quad J_{z}^{(a)} \equiv \frac{1}{2}\left(a_{1}^{\dagger} a_{1}-a_{2}^{\dagger} a_{2}\right)
$$

com expressões correspondentes para átomos da espécie $b$.

Temos então que, neste caso (5.2), (5.3) e (5.4) ficam respectivamente

$$
\begin{aligned}
\mathcal{H}_{a}= & \frac{\left(\mathcal{E}_{a}^{(1)}+\mathcal{E}_{a}^{(2)}\right)}{V} J^{(a)}+\frac{\left(\mathcal{E}_{a}^{(1)}-\mathcal{E}_{a}^{(2)}\right)}{V} J_{z}^{(a)}+\frac{\eta_{a}}{V}\left(J_{+}^{(a)}+J_{-}^{(a)}\right)+ \\
& +\frac{\lambda_{a}^{(1)}}{2 V^{2}}\left(J^{(a)}+J_{z}^{(a)}\right)\left(J^{(a)}+J_{z}^{(a)}-1\right)+\frac{\lambda_{a}^{(2)}}{2 V^{2}}\left(J^{(a)}-J_{z}^{(a)}\right)\left(J^{(a)}-J_{z}^{(a)}-1\right),
\end{aligned}
$$




$$
\begin{aligned}
\mathcal{H}_{b}= & \frac{\left(\mathcal{E}_{b}^{(1)}+\mathcal{E}_{b}^{(2)}\right)}{V} J^{(b)}+\frac{\left(\mathcal{E}_{b}^{(1)}-\mathcal{E}_{b}^{(2)}\right)}{V} J_{z}^{(b)}+\frac{\eta_{b}}{V}\left(J_{+}^{(b)}+J_{-}^{(b)}\right)+ \\
& +\frac{\lambda_{b}^{(1)}}{2 V^{2}}\left(J^{(b)}+J_{z}^{(b)}\right)\left(J^{(b)}+J_{z}^{(b)}-1\right)+\frac{\lambda_{b}^{(2)}}{2 V^{2}}\left(J^{(b)}-J_{z}^{(b)}\right)\left(J^{(b)}-J_{z}^{(b)}-1\right)
\end{aligned}
$$

e

$$
\mathcal{H}_{a b}=\frac{\lambda_{a b}^{(1)}}{V^{2}}\left(J^{(a)}+J_{z}^{(a)}\right)\left(J^{(b)}+J_{z}^{(b)}\right)+\frac{\lambda_{a b}^{(2)}}{V^{2}}\left(J^{(a)}-J_{z}^{(a)}\right)\left(J^{(b)}-J_{z}^{(b)}\right) .
$$

onde $J^{(a, b)} \equiv N_{a, b} / 2$.

Novamente com o objetivo de tomar o limite termodinâmico, torna-se mais conveniente trabalhar com um dos Hamiltonianos escalonados $h^{\left(J_{a}\right)} \equiv V \mathcal{H} / J_{a}$ ou $h^{\left(J_{b}\right)} \equiv V \mathcal{H} / J_{b}$ (por simplicidade, $J_{a} \equiv J^{(a)}$ e $\left.J_{b} \equiv J^{(b)}\right)$. Note que é indiferente se escolhemos trabalhar com $h^{\left(J_{a}\right)}$ ou $h^{\left(J_{b}\right)}$, pois no limite termodinâmico temos $J_{a} \rightarrow \infty$ e $J_{b} \rightarrow \infty$ com $J_{a} / J_{b}=$ constante. Considerando então $h^{\left(J_{a}\right)}$ e definindo $R=J_{b} / J_{a}$ temos que (5.6), (5.7) e (5.8) ficam

$$
\begin{aligned}
\frac{V \mathcal{H}_{a}}{J_{a}}=\mathcal{E}_{a}^{(1)}+ & \mathcal{E}_{a}^{(2)}+\left(\mathcal{E}_{a}^{(1)}-\mathcal{E}_{a}^{(2)}\right) \frac{J_{z}^{(a)}}{J_{a}}+\frac{n_{a} \lambda_{a}^{(1)}}{4}\left(1+\frac{J_{z}^{(a)}}{J_{a}}\right)\left(1+\frac{J_{z}^{(a)}}{J_{a}}-\frac{1}{J_{a}}\right)+ \\
& +\frac{n_{a} \lambda_{a}^{(2)}}{4}\left(1-\frac{J_{z}^{(a)}}{J_{a}}\right)\left(1-\frac{J_{z}^{(a)}}{J_{a}}-\frac{1}{J_{a}}\right)+2 \eta_{a} \frac{J_{x}^{(a)}}{J_{a}} \\
\frac{1}{R} \frac{V \mathcal{H}_{b}}{J_{a}}=\mathcal{E}_{b}^{(1)}+ & \mathcal{E}_{b}^{(2)}+\left(\mathcal{E}_{b}^{(1)}-\mathcal{E}_{b}^{(2)}\right) \frac{J_{z}^{(b)}}{J_{b}}+\frac{n_{b} \lambda_{b}^{(1)}}{4}\left(1+\frac{J_{z}^{(b)}}{J_{b}}\right)\left(1+\frac{J_{z}^{(b)}}{J_{b}}-\frac{1}{J_{b}}\right)+ \\
& +\frac{n_{b} \lambda_{b}^{(2)}}{4}\left(1-\frac{J_{z}^{(b)}}{J_{b}}\right)\left(1-\frac{J_{z}^{(b)}}{J_{b}}-\frac{1}{J_{b}}\right)+2 \eta_{b} \frac{J_{x}^{(b)}}{J_{b}}
\end{aligned}
$$

e

$$
\frac{1}{R} \frac{V \mathcal{H}_{a b}}{J_{a}}=\frac{n_{a} \lambda_{a b}^{(1)}}{2}\left(1+\frac{J_{z}^{(a)}}{J_{a}}\right)\left(1+\frac{J_{z}^{(b)}}{J_{b}}\right)+\frac{n_{a} \lambda_{a b}^{(2)}}{2}\left(1-\frac{J_{z}^{(a)}}{J_{a}}\right)\left(1-\frac{J_{z}^{(b)}}{J_{b}}\right)
$$

onde $n_{a, b}=2 J_{a, b} / V$ e $J_{x}^{(a, b)}=\frac{1}{2}\left(J_{+}^{(a, b)}+J_{-}^{(a, b)}\right)$. 
O limite termodinâmico neste caso consiste em fazer $J_{a, b} \rightarrow \infty$ com $n_{a, b}$ constantes. Com isso temos então que a Hamiltoniana clássica fica

$$
\begin{gathered}
h_{c}^{\left(J_{a}\right)}=\mathcal{E}_{a}^{(1)}+\mathcal{E}_{a}^{(2)}+\left(\mathcal{E}_{a}^{(1)}-\mathcal{E}_{a}^{(2)}\right) j_{z}^{(a)}+\frac{n_{a} \lambda_{a}^{(1)}}{4}\left(1+j_{z}^{(a)}\right)^{2}+\frac{n_{a} \lambda_{a}^{(2)}}{4}\left(1-j_{z}^{(a)}\right)^{2}+2 \eta_{a} j_{x}^{(a)}+ \\
+R\left[\mathcal{E}_{b}^{(1)}+\mathcal{E}_{b}^{(2)}+\left(\mathcal{E}_{b}^{(1)}-\mathcal{E}_{b}^{(2)}\right) j_{z}^{(b)}+\frac{n_{b} \lambda_{b}^{(1)}}{4}\left(1+j_{z}^{(b)}\right)^{2}+\frac{n_{b} \lambda_{b}^{(2)}}{4}\left(1-j_{z}^{(b)}\right)^{2}+2 \eta_{b} j_{x}^{(b)}\right]+ \\
+R\left[\frac{n_{a} \lambda_{a b}^{(1)}}{2}\left(1+j_{z}^{(a)}\right)\left(1+j_{z}^{(b)}\right)+\frac{n_{a} \lambda_{a b}^{(2)}}{2}\left(1-j_{z}^{(a)}\right)\left(1-j_{z}^{(b)}\right)\right] .
\end{gathered}
$$

De posse da Hamiltoniana clássica, é possível obter as equações canônicas de movimento para o sistema. Definindo $j_{a, b} \equiv j_{z}^{(a, b)}$ e $E_{a, b}^{ \pm} \equiv \mathcal{E}_{a, b}^{(1)} \pm \mathcal{E}_{a, b}^{(2)}$ e utilizando a relação $j_{x}^{(a, b)}=\sqrt{1-j_{a, b}^{2}} \cos \varphi_{a, b}$, temos que as equações de movimento, fazendo uma analogia com (4.17), ficam então

$$
\begin{aligned}
& \text { (a) }: \frac{d \varphi_{a}}{d t}=\frac{1}{\hbar} \frac{\partial h_{c}^{\left(J_{a}\right)}}{\partial j_{a}}=\frac{E_{a}^{-}}{\hbar}+\frac{n_{a} \lambda_{a}^{(1)}}{2 \hbar}\left(1+j_{a}\right)+\frac{n_{a} \lambda_{a}^{(2)}}{2 \hbar}\left(1-j_{a}\right)+ \\
& +R\left[\frac{n_{a} \lambda_{a b}^{(1)}}{2 \hbar}\left(1+j_{b}\right)-\frac{n_{a} \lambda_{a b}^{(2)}}{2 \hbar}\left(1-j_{b}\right)\right]-\frac{2 \eta_{a}}{\hbar} \frac{j_{a}}{\sqrt{1-j_{a}^{2}}} \cos \varphi_{a}, \\
& \text { (b) : } \frac{d j_{a}}{d t}=-\frac{1}{\hbar} \frac{\partial h_{c}^{\left(J_{a}\right)}}{\partial \varphi_{a}}=\frac{2 \eta_{a}}{\hbar} \sqrt{1-j_{a}^{2}} \sin \varphi_{a} \\
& \text { (c) : } \frac{d \varphi_{b}}{d t}=\frac{1}{\hbar} \frac{\partial h_{c}^{\left(J_{a}\right)}}{\partial j_{b}}=\frac{E_{b}^{-}}{\hbar}+\frac{n_{b} \lambda_{b}^{(1)}}{2 \hbar}\left(1+j_{b}\right)+\frac{n_{b} \lambda_{b}^{(2)}}{2 \hbar}\left(1-j_{b}\right)+ \\
& +R\left[\frac{n_{a} \lambda_{a b}^{(1)}}{2 \hbar}\left(1+j_{a}\right)-\frac{n_{a} \lambda_{a b}^{(2)}}{2 \hbar}\left(1-j_{a}\right)\right]-\frac{2 \eta_{b} R}{\hbar} \frac{j_{b}}{\sqrt{1-j_{b}^{2}}} \cos \varphi_{b}, \\
& \text { (d) : } \frac{d j_{b}}{d t}=-\frac{1}{\hbar} \frac{\partial h_{c}^{\left(J_{a}\right)}}{\partial \varphi_{b}}=\frac{2 \eta_{b} R}{\hbar} \sqrt{1-j_{b}^{2}} \sin \varphi_{b} .
\end{aligned}
$$

Com as equações de movimento (5.13), podemos enfim estudar o comportamento temporal do sistema. Como primeiro passo, seria interessante encontrar os pontos de equilíbrio do sistema. Para tanto, procuraremos os pontos onde as equações $(5.13 b)$ e $(5.13 d)$ se anulam $\left(d j_{a . b} / d t=0\right)$, ou seja,

$$
\frac{2 \eta_{a}}{\hbar} \sqrt{1-j_{a}^{2}} \sin \varphi_{a}=0 \quad \text { e } \quad \frac{2 \eta_{b} R}{\hbar} \sqrt{1-j_{b}^{2}} \sin \varphi_{b}=0
$$

que nos fornecem como possíveis soluções $j_{a, b}= \pm 1$ ou $\varphi_{a, b}=k \pi, k \in \mathbb{Z}$.

Para $j_{a, b}= \pm 1$ vemos que ocorrem problemas de divergência nos últimos termos das equações $(5.13 a)$ e $(5.13 c)$, o que nos leva a concluir que as soluções interessantes são $\varphi_{a, b}=k \pi, k \in \mathbb{Z}$. Portanto, se quisermos encontrar os pontos de equilíbrio do sistema, será necessário procurar pelas 
soluções do seguinte sistema de equações acopladas

$$
\begin{aligned}
& (a): \frac{E_{a}^{-}}{\hbar}+\frac{n_{a} \lambda_{a}^{(1)}}{2 \hbar}\left(1+j_{a}\right)+\frac{n_{a} \lambda_{a}^{(2)}}{2 \hbar}\left(1-j_{a}\right)+R\left[\frac{n_{a} \lambda_{a b}^{(1)}}{2 \hbar}\left(1+j_{b}\right)-\frac{n_{a} \lambda_{a b}^{(2)}}{2 \hbar}\left(1-j_{b}\right)\right]+ \\
& \quad-\frac{2 \eta_{a}}{\hbar} \frac{j_{a}}{\sqrt{1-j_{a}^{2}}}( \pm 1)=0 \\
& (b): \frac{E_{b}^{-}}{\hbar}+\frac{n_{b} \lambda_{b}^{(1)}}{2 \hbar}\left(1+j_{b}\right)+\frac{n_{b} \lambda_{b}^{(2)}}{2 \hbar}\left(1-j_{b}\right)+R\left[\frac{n_{a} \lambda_{a b}^{(1)}}{2 \hbar}\left(1+j_{a}\right)-\frac{n_{a} \lambda_{a b}^{(2)}}{2 \hbar}\left(1-j_{a}\right)\right]+ \\
& \quad-\frac{2 \eta_{b} R}{\hbar} \frac{j_{b}}{\sqrt{1-j_{b}^{2}}}( \pm 1)=0 .
\end{aligned}
$$

Para tanto, adotaremos unidades tais que $n_{a}=1$ e $\hbar=1$; além disso tomaremos para os parâmetros os seguintes valores teste $R=1$ (que implica $n_{b}=n_{a}$ ), $\lambda_{a}^{(1)}=\lambda_{a}^{(2)}=1, \lambda_{b}^{(1)}=\lambda_{b}^{(2)}=1$, $\lambda_{a b}^{(1)}=\lambda_{a b}^{(2)}=1, \mathcal{E}_{a}^{(1)}=\mathcal{E}_{a}^{(2)} \equiv 0$ e $\mathcal{E}_{b}^{(1)}=\mathcal{E}_{b}^{(2)} \equiv 0$. Com estes valores, os seguintes pontos aparecem como solução, quando anulamos as derivadas temporais em (5.13),

\begin{tabular}{|c|c|c|c|c|}
\hline Ponto & $\varphi_{a}$ & $\varphi_{b}$ & $j_{a}$ & $j_{b}$ \\
\hline \hline 1 & 0 & 0 & 0.632293 & 0.632293 \\
\hline 2 & 0 & $\pi$ & 0.231399 & -0.524292 \\
\hline 3 & $\pi$ & 0 & -0.524292 & 0.231399 \\
\hline 4 & $\pi$ & $\pi$ & -0.321336 & -0.321336 \\
\hline
\end{tabular}

Ao aplicarmos o teste da segunda derivada (autovalores da matriz Hessiana), vemos que os pontos 1 e 4 são respectivamente pontos de máximo e mínimo, enquanto que os pontos 2 e 3 são ambos pontos de sela. Este fato fica mais claro através da figura 5.1. Nela podemos ver o comportamento de $h_{c}^{\left(J_{a}\right)}$ na vizinhança de cada um dos pontos da tabela. 

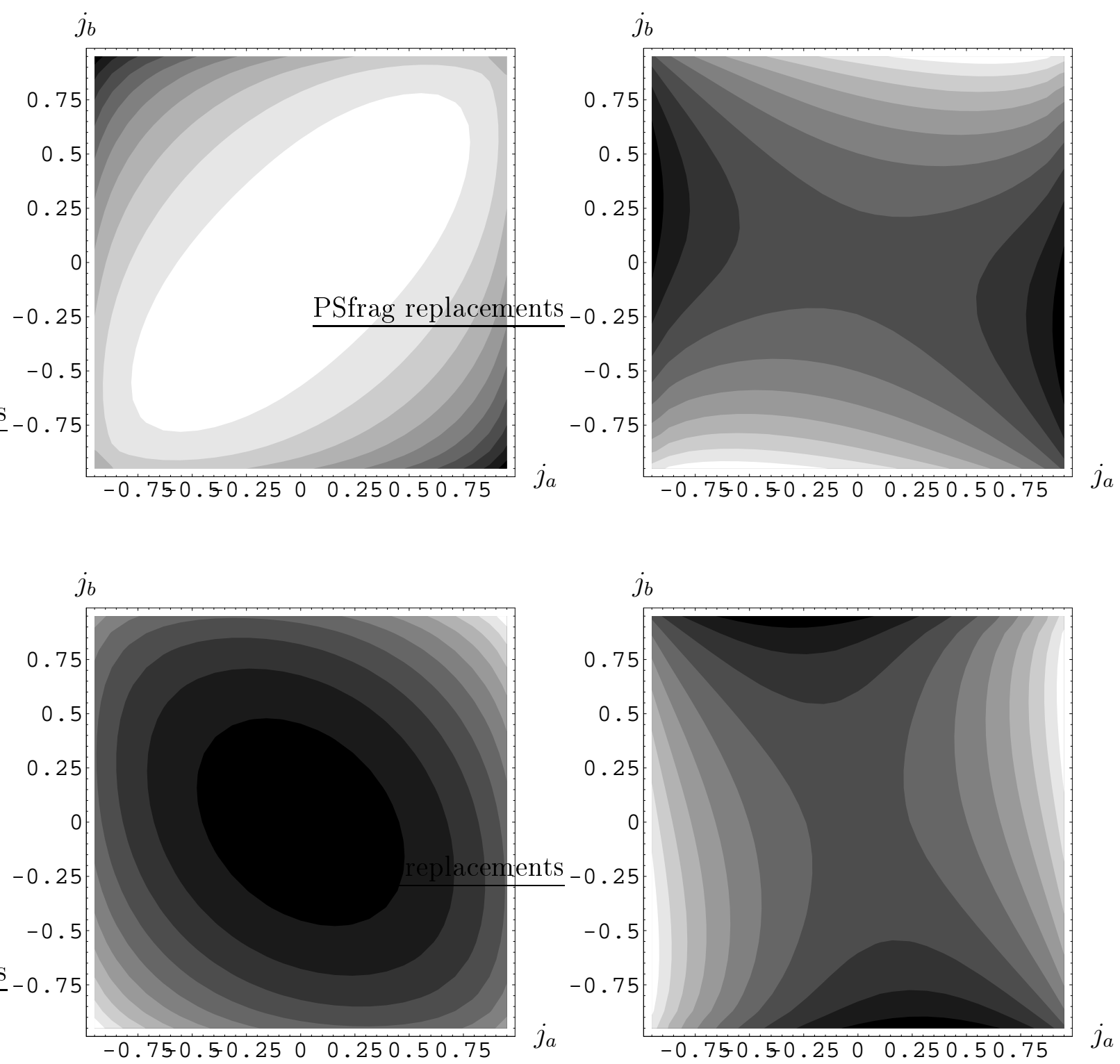

Figura 5.1: Contornos de $h_{c}^{\left(J_{a}\right)}$ por $\left(j_{a}, j_{b}\right)$, para $\left(\varphi_{a}, \varphi_{b}\right)=(0,0),(0, \pi),(\pi, 0)$ e $(\pi, \pi)$, respectivamente no sentido horário.

A existência de pontos de equilíbrio instável (pontos de sela 2 e 3) nos fornece mais um indício de que o sistema (5.1) pode apresentar um comportamento irregular. Contudo, já é possível encontrar um comportamento bastante complexo em uma versão mais simples de (5.1). De fato, como mencionamos anteriormente, este sistema se reduz ao sistema estudado por [56], a saber (5.20), cujo limite clássico apresenta um comportamento caótico para um determinado valor do parâmetro de acoplamento $\mu$. Este fato será explorado na seção seguinte. 


\subsection{Sistema de Dois Momentos Angulares Acoplados}

\subsubsection{Hamiltoniano do Sistema e seu Limite Clássico}

Uma maneira mais direta de verificar que o sistema (5.1) apresenta, em determinadas condições, um comportamento irregular é, como mencionado anteriormente, utilizar o fato de que este sistema contém o sistema de dois momentos angulares acoplados estudado em [56].

A forma mais simples do Hamiltoniano que descreve um sistema de dois momentos angulares acoplados é uma generalização do caso $N=2$ do modelo de Ising com campo transversal, i.e.,

$$
\hat{H}=\omega \hat{J}_{x} \otimes \hat{I}+\omega \hat{I} \otimes \hat{J}_{x}+\frac{\chi}{j} \hat{J}_{z} \otimes \hat{J}_{z}
$$

onde o operador de momento angular $\hat{J}_{i}$ satisfaz às relações de comutação de $S U(2),\left[\hat{J}_{x}, \hat{J}_{y}\right]=\mathrm{i} \hat{J}_{z}$ (e permutações cíclicas). Definindo $\mu=\chi / \omega$, obtemos um Hamiltoniano a um parâmetro

$$
\hat{H}=\hat{J}_{x, 1}+\hat{J}_{x, 2}+\frac{\mu}{j} \hat{J}_{z, 1} \hat{J}_{z, 2}
$$

onde utilizamos a notação $\hat{J}_{i, 1}=\hat{J}_{i} \otimes \hat{I}$ e $\hat{J}_{i, 2}=\hat{I} \otimes \hat{J}_{i}, i=x, y, z$, de tal forma que o índice 1 (2) se refere ao subsistema $1(2)$.

Os quadrados do momento angular total dos subsistemas individuais,

$$
\hat{J}_{1(2)}^{2}=\hat{J}_{x, 1(2)}^{2}+\hat{J}_{y, 1(2)}^{2}+\hat{J}_{z, 1(2)}^{2},
$$

satisfazem às relações de comutação

$$
\left[\hat{J}_{1}^{2}, \hat{H}\right]=\left[\hat{J}_{2}^{2}, \hat{H}\right]=0
$$

e portanto são constantes de movimento. Em [56], foram considerados momentos angulares idênticos, de tal forma que $\hat{J}_{1}^{2}=\hat{J}_{2}^{2}=j(j+1)$. Isto nos permite representar o sistema numa base formada pelo produto de autoestados de $\hat{J}_{z}$, tal que $|j, m\rangle \otimes|j, n\rangle \equiv|m, n\rangle$, onde $-j \leq m, n \leq j$.

Note que o termo de acoplamento no Hamiltoniano é escalonado com $j$, afim de tomarmos o limite clássico, que neste caso corresponde ao limite $j \rightarrow \infty$. Para obter a Hamiltoniana clássica, definimos as coordenadas $L_{i, 1(2)}=\left\langle\hat{J}_{i, 1(2)}\right\rangle / j$, que no limite clássico permite a fatoração dos momentos, i.e., $\left\langle\hat{J}_{x, 1} \hat{J}_{z, 1}\right\rangle / j^{2} \rightarrow L_{x, 1} L_{z, 1}$ (ver o Apêndice de [56] para maiores detalhes). O Hamiltoniana clássica correspondente fica então

$$
E=L_{x, 1}+L_{x, 2}+\mu L_{z, 1} L_{z, 2}
$$

onde $E=\langle\hat{H}\rangle / j$, com o vínculo $L_{x, 1(2)}^{2}+L_{y, 1(2)}^{2}+L_{z, 1(2)}^{2}=1$.

Segundo [56], a análise do sistema clássico correspondente ao Hamiltoniano (5.18), mostrou 
que a não-linearidade do termo de interação leva à existência de comportamento caótico para um determinado intervalo do valor dos parâmetros e certas condições iniciais.

\subsubsection{Equivalência Entre o Modelo de Dois Momentos Angulares Aco- plados e o Modelo (5.1)}

Se considerarmos na Hamiltoniana clássica (5.12) unidades tais que $n_{a}=1$ e o conjunto de parâmetros $J_{a}=J_{b}=J$ (que implica $R=1$ e $n_{b}=n_{a}$ ), $\eta_{a}=\eta_{b}=1 / 2, \lambda_{a}^{(1)}=\lambda_{a}^{(2)}=0, \lambda_{b}^{(1)}=\lambda_{b}^{(2)}=0$, $\lambda_{a b}^{(1)}=\lambda_{a b}^{(2)}=\mu, \mathcal{E}_{a}^{(1)}=\mathcal{E}_{a}^{(2)}=\mathcal{E}_{a}, \mathcal{E}_{b}^{(1)}=\mathcal{E}_{b}^{(2)}=\mathcal{E}_{b}$ e $\mathcal{E}_{a}+\mathcal{E}_{b}=-\mu / 2$, temos que (5.12) fica

$$
h_{c}^{(J)}=j_{x}^{(a)}+j_{x}^{(b)}+\mu j_{z}^{(a)} j_{z}^{(b)}
$$

que como podemos ver possui a mesma forma de (5.18).

Segundo [56], para valores do parâmetro de acoplamento $\mu$ abaixo de um dado valor crítico $\mu_{c}=1$ o sistema (5.19) apresenta uma dinâmica predominantemente regular, enquanto que para valores de $\mu$ acima de $\mu_{c}$ o espaço de fases do sistema apresenta extensas regiões de comportamento caótico. Este comportamento pode ser confirmado numericamente através da seção de Poincaré de (5.19) mostrada na figura 5.2.

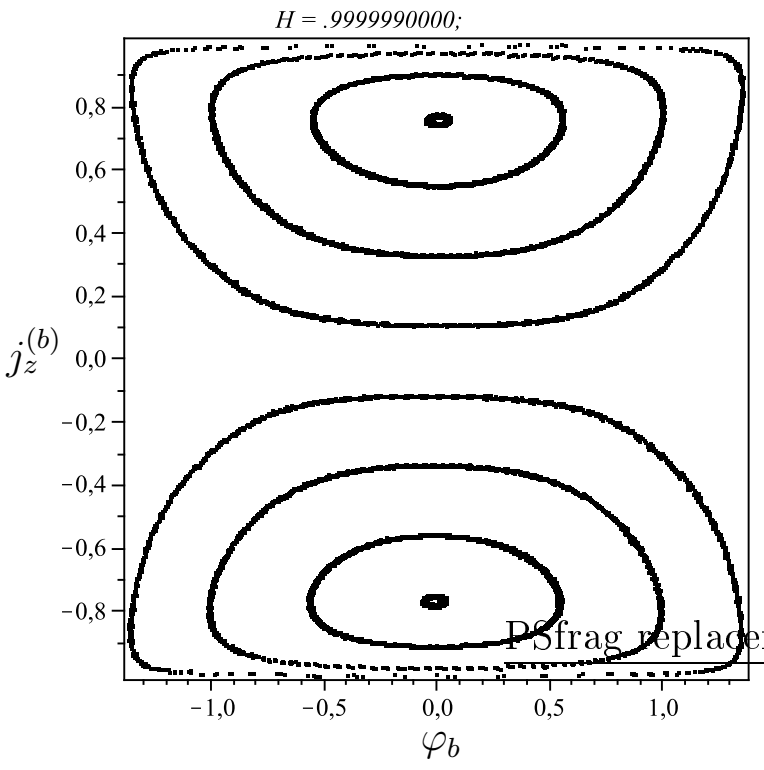

(a)

\section{Figura 5.2: Seção de Poincaré $\left(\cdot(b), \varphi_{b}\right)$}

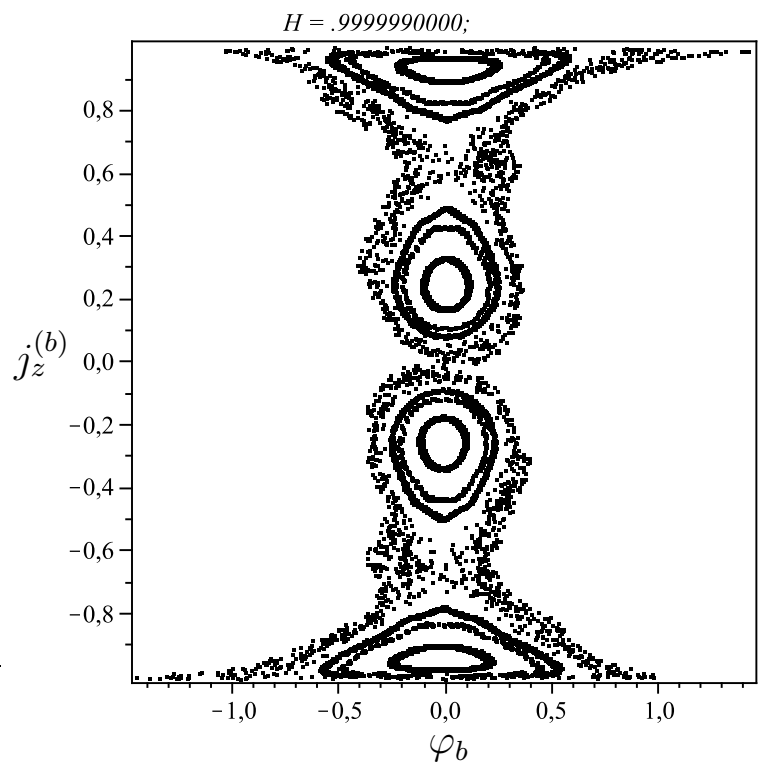

(b)

Vemos portanto que o sistema descrito por (5.20) é dotado de propriedades muito interessantes e pertinentes ao nosso estudo. Este sistema, além de possuir propensão coletiva, apresenta um comportamento no limite clássico que pode ser regular ou caótico, dependendo apenas do valor do parâmetro de acoplamento $\mu$, o que nos permite comparar os dois casos. Com isso, passaremos 
na próxima seção ao estudo da heterogeneidade distribucional no espaço de fases $\tilde{h}_{q}(\hat{\rho})$, para este sistema.

\subsection{Heterogeneidade Distribucional para o Modelo de Dois Momentos Angulares Acoplados}

Tomando como base a equivalência, sob determinadas condições, que existe entre os modelos (5.12) e (5.18) e que foi descrita na subseção 5.2.2, estudaremos na presente seção o comportamento da heterogeneidade distribucional quântica no espaço de fases $\tilde{h}_{q}(\hat{\rho})$, não para o modelo $(5.1)$ mas sim para o modelo

$$
H=J_{x}^{(a)}+J_{x}^{(b)}+\frac{\mu}{J} J_{z}^{(a)} J_{z}^{(b)}
$$

onde os $J_{x, z}^{(a, b)}$ e $J$ são os operadores coletivos (ou de quasi-spin) definidos em (5.5). Como podemos ver, o modelo (5.20) possui uma forma mais simples sendo portanto bem mais simples de ser tratado do que o modelo (5.1). Além disso, o limite $J \rightarrow \infty$ do Hamiltoniano escalonado $H / J$ (limite clássico) é dado exatamente por (5.19), cujo comportamento é bem conhecido (ver [56]). A utilização de (5.20) nos possibilitará então estudar a influência que a regularidade ou não do sistema no limite clássico tem sobre o comportamento da heterogeneidade distribucional quântica, ou seja, sobre o comportamento de $\tilde{h}_{q}(\hat{\rho})$.

Para que possamos estudar o comportamento de $\tilde{h}_{q}(\hat{\rho})$ para o sistema $(5.20)$, precisamos extender a definição (3.39), da média (coarse-graining) de um operador densidade $\hat{\rho}$, para o caso de sistemas com dois graus de liberdade. Considerando novamente que os dois subsistemas $(a, b)$ são idênticos, podemos representar o espaço de Hilbert $\mathcal{H}$ do sistema completo como o produto direto dos espaços de Hilbert de cada um dos subsistemas $\mathcal{H}_{i=a, b}$, ou seja, $\mathcal{H} \equiv \mathcal{H}_{a} \otimes \mathcal{H}_{a}$. Desta forma,

podemos fazer uma analogia com as definições (3.1) - (3.6) e construir operadores unitários $\hat{U}_{a, b}$ e $\hat{V}_{a, b}$ de tal forma que

$$
\begin{aligned}
& \hat{U}_{a}\left|v_{a}^{k_{a}} v_{b}^{k_{b}}\right\rangle=\left|v_{a}^{k_{a}+1} v_{b}^{k_{b}}\right\rangle, \\
& \hat{U}_{b}\left|v_{a}^{k_{a}} v_{b}^{k_{b}}\right\rangle=\left|v_{a}^{k_{a}} v_{b}^{k_{b}+1}\right\rangle, \\
& \hat{V}_{a}\left|u_{a}^{k_{a}} u_{b}^{k_{b}}\right\rangle=\left|u_{a}^{k_{a}-1} u_{b}^{k_{b}}\right\rangle \text {, } \\
& \hat{V}_{b}\left|u_{a}^{k_{a}} u_{b}^{k_{b}}\right\rangle=\left|u_{a}^{k_{a}} u_{b}^{k_{b}-1}\right\rangle,
\end{aligned}
$$

com 


$$
\begin{aligned}
\left|u_{a}^{k_{a}} u_{b}^{k_{b}}\right\rangle & \equiv\left|u_{a}^{k_{a}\left(\bmod D_{a}\right)} u_{b}^{k_{b}\left(\bmod D_{b}\right)}\right\rangle, \\
\left|v_{a}^{k_{a}} v_{b}^{k_{b}}\right\rangle & \equiv\left|v_{a}^{k_{a}\left(\bmod D_{a}\right)} v_{b}^{k_{b}\left(\bmod D_{b}\right)}\right\rangle,
\end{aligned}
$$

onde $D_{j}$ é a dimensão do espaço de Hilbert correspondente ao $j$-ésimo grau de liberdade, $j=a, b$. Segue então de (5.21) e (5.22)que

$$
\hat{V}_{j}^{D_{j}}=\hat{U}_{j}^{D_{j}}=\hat{1}
$$

de onde, juntamente com (5.22), segue que

$$
\begin{aligned}
& \hat{U}_{a}\left|u_{a}^{k_{a}} u_{b}^{k_{b}}\right\rangle=u_{a}^{k_{a}}\left|u_{a}^{k_{a}} u_{b}^{k_{b}}\right\rangle, \\
& \hat{U}_{b}\left|u_{a}^{k_{a}} u_{b}^{k_{b}}\right\rangle=u_{b}^{k_{b}}\left|u_{a}^{k_{a}} u_{b}^{k_{b}}\right\rangle, \\
& \hat{V}_{a}\left|v_{a}^{k_{a}} v_{b}^{k_{b}}\right\rangle=v_{a}^{k_{a}}\left|v_{a}^{k_{a}} v_{b}^{k_{b}}\right\rangle, \\
& \hat{V}_{b}\left|v_{a}^{k_{a}} v_{b}^{k_{a}}\right\rangle=v_{b}^{k_{b}}\left|v_{a}^{k_{a}} v_{b}^{k_{b}}\right\rangle,
\end{aligned}
$$

onde

$$
\begin{aligned}
& u_{a}^{k_{a}}=v_{a}^{k_{a}}=\exp \left(\frac{2 \pi \mathrm{i}}{D_{a}}\right), \\
& u_{b}^{k_{b}}=v_{b}^{k_{b}}=\exp \left(\frac{2 \pi \mathrm{i}}{D_{b}}\right) .
\end{aligned}
$$

Temos também que os $\hat{U}_{a, b}$ e $\hat{V}_{a, b}$ obedecem as relações de comutação

$$
\hat{V}_{i} \hat{U}_{j}=\left\{\begin{array}{ll}
\exp \left(\frac{2 \pi \mathrm{i}}{D_{i}}\right) \hat{U}_{j} \hat{V}_{i} & , \quad i=j \\
\hat{U}_{j} \hat{V}_{i} & , \quad i \neq j
\end{array} ;\right.
$$

e que os estados $\left|u_{a}^{k_{a}} u_{b}^{k_{b}}\right\rangle$ e $\left|v_{a}^{k_{a}} v_{b}^{k_{b}}\right\rangle$ se relacionam através de

$$
\left\langle u_{a}^{k_{a}} u_{b}^{k_{b}} \mid v_{a}^{l_{a}} v_{b}^{l_{b}}\right\rangle=\prod_{j}\left\langle u_{j}^{k_{j}} \mid v_{j}^{l_{j}}\right\rangle
$$

onde

$$
\left\langle u_{j}^{k_{j}} \mid v_{j}^{l_{j}}\right\rangle=D_{j}^{-1 / 2} \exp \left(\frac{2 \pi \mathrm{i}}{D_{j}} k_{j} l_{j}\right)
$$

$\operatorname{com} j=a, b$.

Com as definições (5.21) - (5.24) e fazendo uma analogia com a definição (3.39), temos que a 
média (coarse-graining) sobre o operador densidade $\hat{\rho}$ neste caso será dada por

$$
\hat{\rho}_{\alpha}=\sum_{m_{a}, n_{a}} \sum_{m_{b}, n_{b}} K_{\alpha}\left(m_{a}, n_{a}, m_{b}, n_{b}\right) \hat{U}_{a}^{m_{a}} \hat{V}_{a}^{n_{a}} \hat{U}_{b}^{m_{b}} \hat{V}_{b}^{n_{b}} \hat{\rho} \hat{V}_{b}^{-n_{b}} \hat{U}_{b}^{-m_{b}} \hat{V}_{a}^{-n_{a}} \hat{U}_{a}^{-m_{a}}
$$

onde $K_{\alpha}\left(m_{a}, n_{a}, m_{b}, n_{b}\right)$ é uma função de alisamento quadridimensional. Em nosso estudo consideraremos que esta função possui a forma

$$
K_{\alpha}\left(m_{a}, n_{a}, m_{b}, n_{b}\right)=C \exp \left[-\frac{\left(m_{1}^{2}+n_{1}^{2}+m_{2}^{2}+n_{2}^{2}\right)}{\alpha}\right],
$$

onde $C$ é uma constante de normalização que vem de

$$
\sum_{m_{a}, n_{a} ; m_{b}, n_{b}} K_{\alpha}\left(m_{a}, n_{a}, m_{b}, n_{b}\right)=1
$$

Considerando então, para o modelo com dois graus de liberdade (5.20), um estado inicial dado por

$$
\left|\Psi_{0}\right\rangle \equiv\left|\Psi_{0}^{(a)}\right\rangle \otimes\left|\Psi_{0}^{(b)}\right\rangle
$$

onde

$$
\left|\Psi_{0}^{(a, b)}\right\rangle \equiv\left|\xi_{a, b}, \vartheta_{a, b}\right\rangle_{N_{a, b}}+\left|\xi_{a, b},-\vartheta_{a, b}\right\rangle_{N_{a, b}}
$$

$\operatorname{com}\left|\xi_{i}, \vartheta_{i}\right\rangle_{N_{i}}, i=a, b$, definido em (4.11), ou seja, para cada um dos graus de liberdade do sistema temos uma superposição coerente de dois picos no espaço de fases correspondente.

O ponto do espaço de fases em torno do qual cada um dos picos estará localizado, é um ponto cujas trajetórias oriundas de seu correspondente no espaço de fases clássico apresentam um comportamento regular ou caótico dependendo do valor do parâmetro de acoplamento $\mu$ em (5.19). A figura 5.3 mostra a seção de Poincaré $\left(\varphi_{b}, j_{z}^{(b)}\right)$ no espaço de fases clássico para uma condição inicial oriunda no ponto $j_{z}^{(a)} \approx 0.5555555554, j_{z}^{(b)} \approx-0.7230908941$ e $\varphi_{a}=\varphi_{b}=0$. 


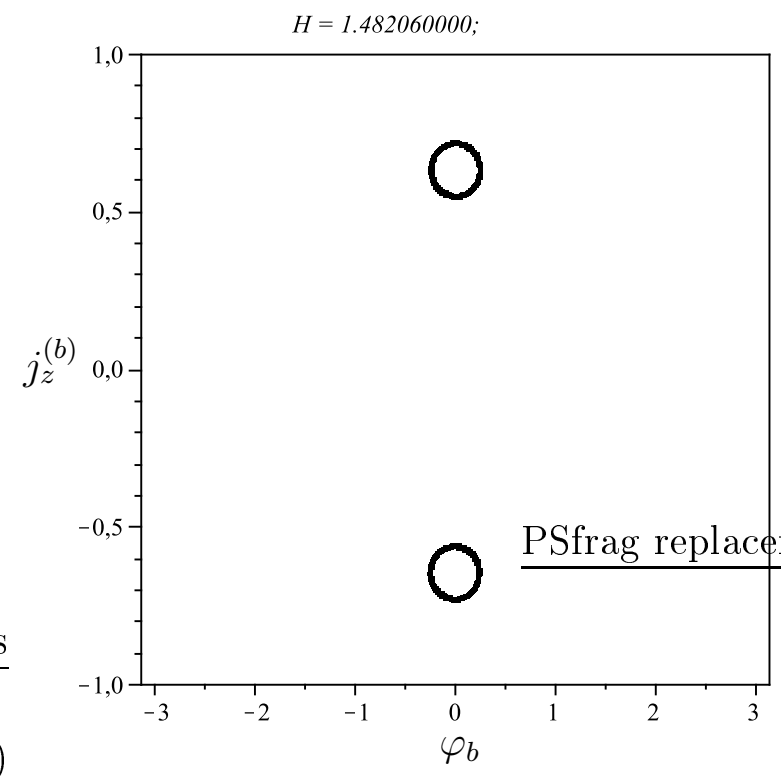

(a)

Figura 5.3: Seção de Poincaré $\left(\varphi_{b}, j_{z}^{(b)}\right)$. Nas figuras a condição inicial é $j_{z}^{(a)} \approx 0.5555555554$, $j_{z}^{(b)} \approx-0.7230908941$ e $\varphi_{a}=\varphi_{b}=0$. Os diferentes gráficos correspondem aos valores do parâmetro $\mu=0.1$ (a) e 1.3 (b).

O comportamento da heterogeneidade distribucional no espaço de fases $\tilde{h}_{q}(\hat{\rho})$ é mostrado na figura 5.4, para uma média (coarse-graining) do tipo (4.12)(2) em cada grau de liberdade. Vale mencionar que com a condição inicial utilizada, o comportamento de $\tilde{h}_{q}(\hat{\rho})$ para o estado inicial (5.26) é equivalente ao do caso em que o estado inicial possui apenas um pico localizado no espaço de fases, e.g. (4.9). 

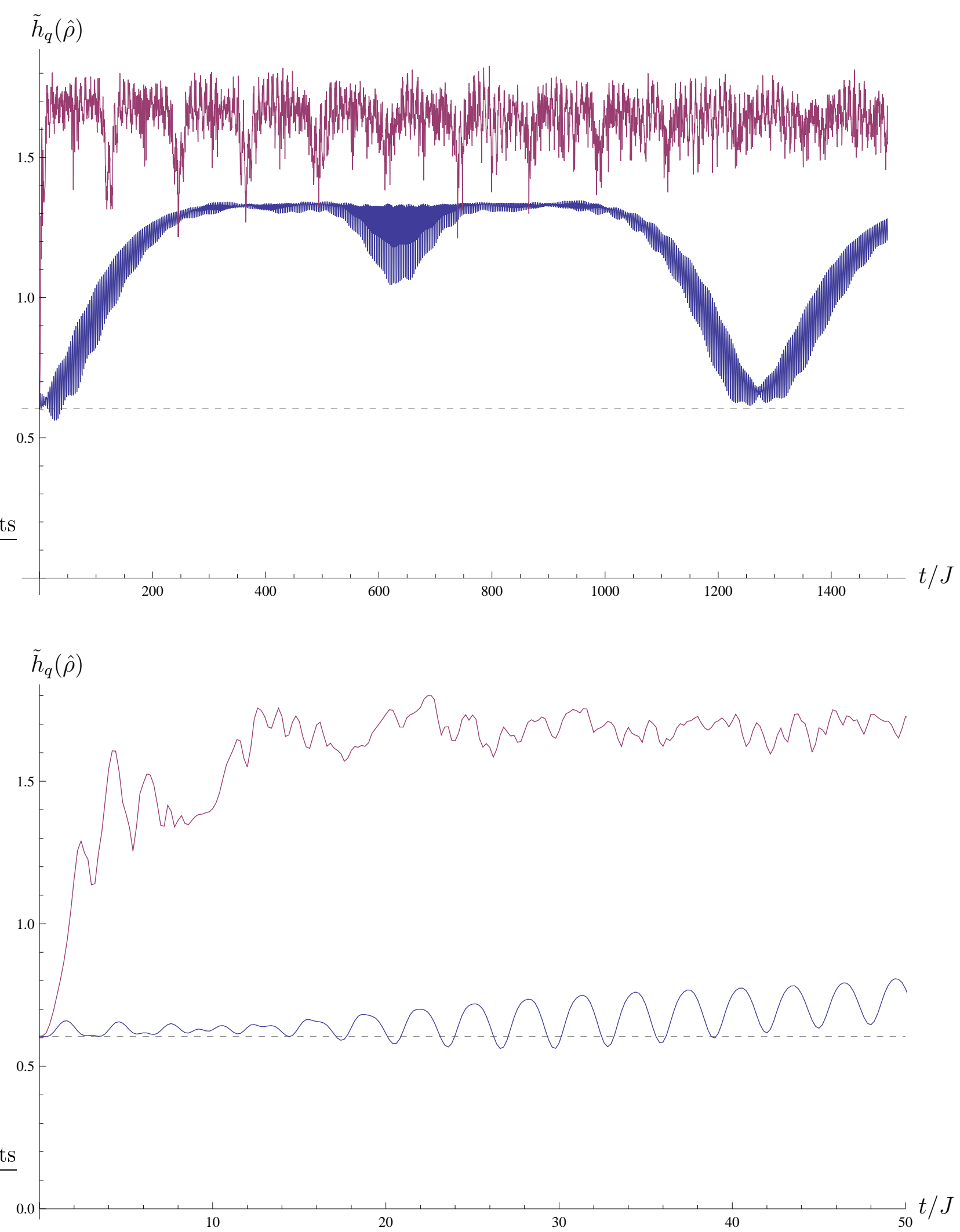

Figura 5.4: Heterogeneidade distribucional no espaço de fases como função do tempo escalonado $t / J$, para $N_{a}=N_{b}=10, \alpha=0.25$ e $M=3$. As diferentes cores referem-se aos diferentes valores do parâmetro $\mu=0.1$ (azul) e 1.3 (vermelho). A linha tracejada horizontal corresponde ao valor inicial de $\tilde{h}_{q}(\hat{\rho}) \approx 0.605274$. 
Na figura 5.3 vemos que o perfil do espaço de fases, para a condição inicial dada, muda drasticamente com o valor do parâmetro de acoplamento $\mu$, indo de um comportamento totalmente regular $(\mu=0.1)$ no gráfico do topo, até um comportamento caótico $(\mu=1.3)$ no outro gráfico. Uma variação igualmente drástica também pode ser notada no comportamento da heterogeneidade distribucional no espaço de fases na figura 5.4. Nesta figura vemos que o comportamento inicial de $\tilde{h}_{q}(\hat{\rho})$ está de pleno acordo com os resultados da literatura [16, 18, 19], ou seja, o aumento da estrutura da distribuição no espaço de fases ocorre bem mais rapidamente na situação em que o sistema é classicamente caótico. Conforme o sistema evolui no tempo, vemos que aparece um patamar de saturação no comportamento de $\tilde{h}_{q}(\hat{\rho})$ no caso caótico, enquanto que no caso regular observamos um comportamento (quase) periódico com grandes recorrências (oscilações) no valor de $\tilde{h}_{q}(\hat{\rho})$, o que está em pleno acordo com a literatura, como vimos para o modelo de Bose-Hubbard com dois sítios e $N$ bósons (ver figura 4.12). O ponto novo que podemos observar na figura 5.4 é que a amplitude máxima da oscilação de $\tilde{h}_{q}(\hat{\rho})$ no caso classicamente integrável está localizada bem abaixo do patamar de saturação de $\tilde{h}_{q}(\hat{\rho})$ no caso classicamente caótico, indicando que neste caso a distribuição adquire mais estrutura em pequenas escalas do espaço de fases do que no caso integrável.

Assim como para o modelo $H_{B H_{2}}$ em (4.3), temos neste modelo que $\tilde{h}_{q}(\hat{\rho})$ também possui uma série de "sub-períodos" para o caso classicamente integrável, e como mencionamos naquela ocasião (ver o último parágrafo da subseção 4.2.1), este fato está diretamente relacionado à distribuição dos autovalores de energia. Isto fica mais claro através da figura 5.5.

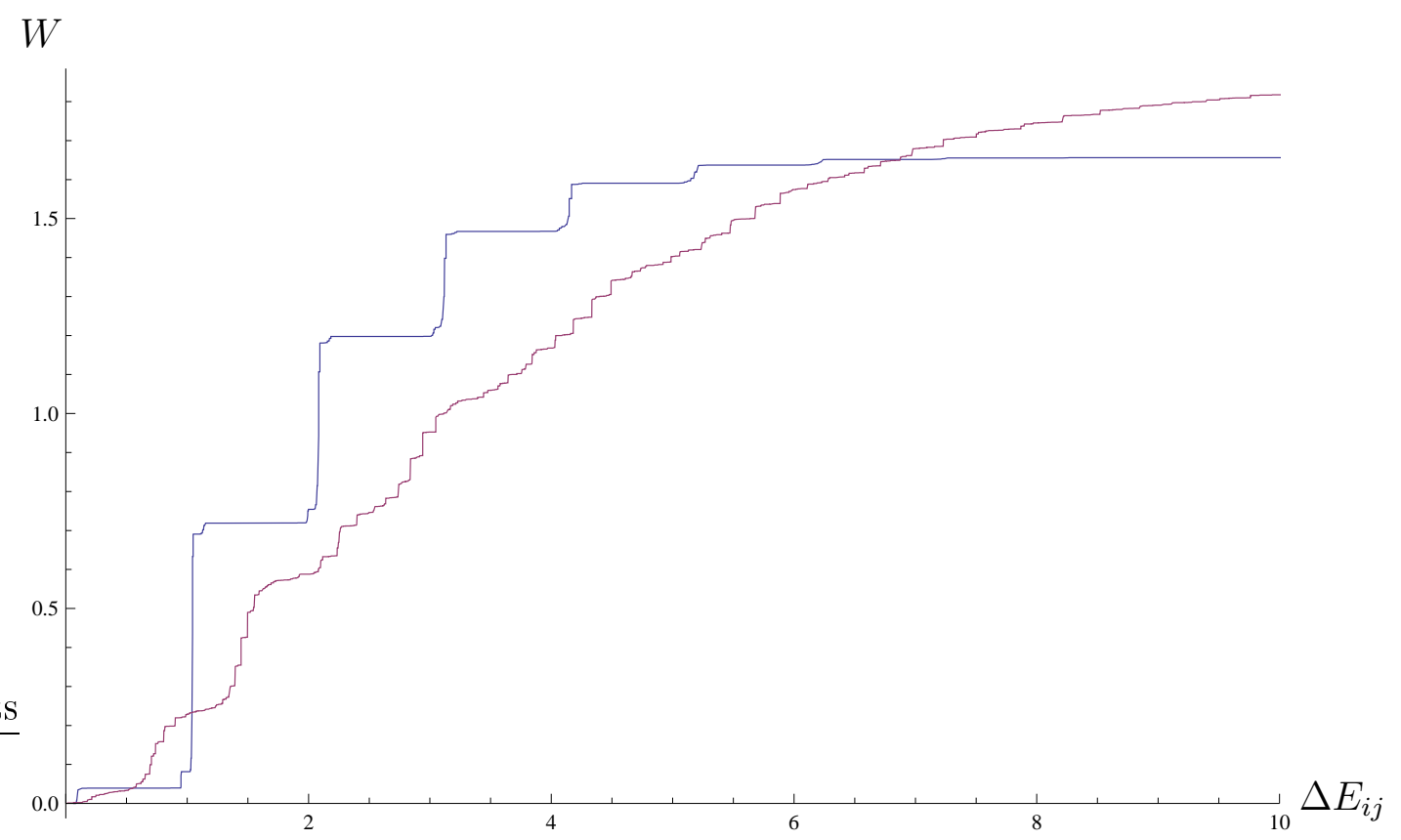

Figura 5.5: Distribuição das diferenças de energia $\Delta E_{i j}=\left|E_{i}-E_{j}\right| \operatorname{com} i, j=1, \ldots, N+1$, pelos respectivos pesos $W=\sum_{\Delta E_{k l}<\Delta E_{i j}} 4 c_{k} c_{l}$, com $c_{n}=\left\langle E_{n} \mid \Psi_{0}\right\rangle, E_{n}$ e $\left|E_{n}\right\rangle$ sendo respectivamente os autovalores e autoestados do Hamiltoniano (5.20) e $\left|\Psi_{0}\right\rangle$ dado por (5.26). Os valores dos parâmetros e a descrição das diferentes cores são os mesmos da figura 5.4. 
Vemos claramente na figura 5.5, que a distribuição das diferenças de energia $\Delta E_{i j}$ depende drasticamente se o sistema é classicamente integrável ou caótico, sendo esta muito mais regular no primeiro caso. Como a uma dada diferença de energia $\Delta E_{i j}$ está associada uma frequência $\Delta E_{i j} / \hbar$, podemos inferir que a regularidade em $\tilde{h}_{q}(\hat{\rho})$ e a regularidade em $\Delta E_{i j}$ estão de alguma forma relacionadas. Esta suspeita é corroborada pelo fato da regularidade ser perdida no caso classicamente caótico tanto para $\tilde{h}_{q}(\hat{\rho})$ como para $\Delta E_{i j}$. No entanto, não foi possível determinar de maneira clara a forma como os dois comportamentos estão relacionados. Por exemplo, não conseguimos fazer uma associação direta entre um dado período (dentre os vários) do comportamento temporal de $\tilde{h}_{q}(\hat{\rho})$ e propriedades na distribuição dos autovalores de energia.

As figuras 5.4 e 5.5 nos mostram claramente o papel que o caos no sistema clássico desempenha, tanto com relação à variação de estrutura na distribuição no espaço de fases quanto com relação à distribuição dos autovalores de energia do sistema quântico. No entanto, do ponto de vista de correspondência quântico-clássica elas nos dizem muito pouco. Um pouco mais elucidativa é a figura 5.6, que nos mostra uma comparação entre os comportamentos de $\tilde{h}_{q}(\hat{\rho})$ para $N_{a}=N_{b}=10$ e 20. Vemos novamente que os resultados estão de acordo com os resultados da literatura, uma vez que a curva correspondente ao caso mais próximo do limite clássico, ou seja, com maior valor de $N$, está posicionada abaixo da curva correpondente ao menor valor de $N$.

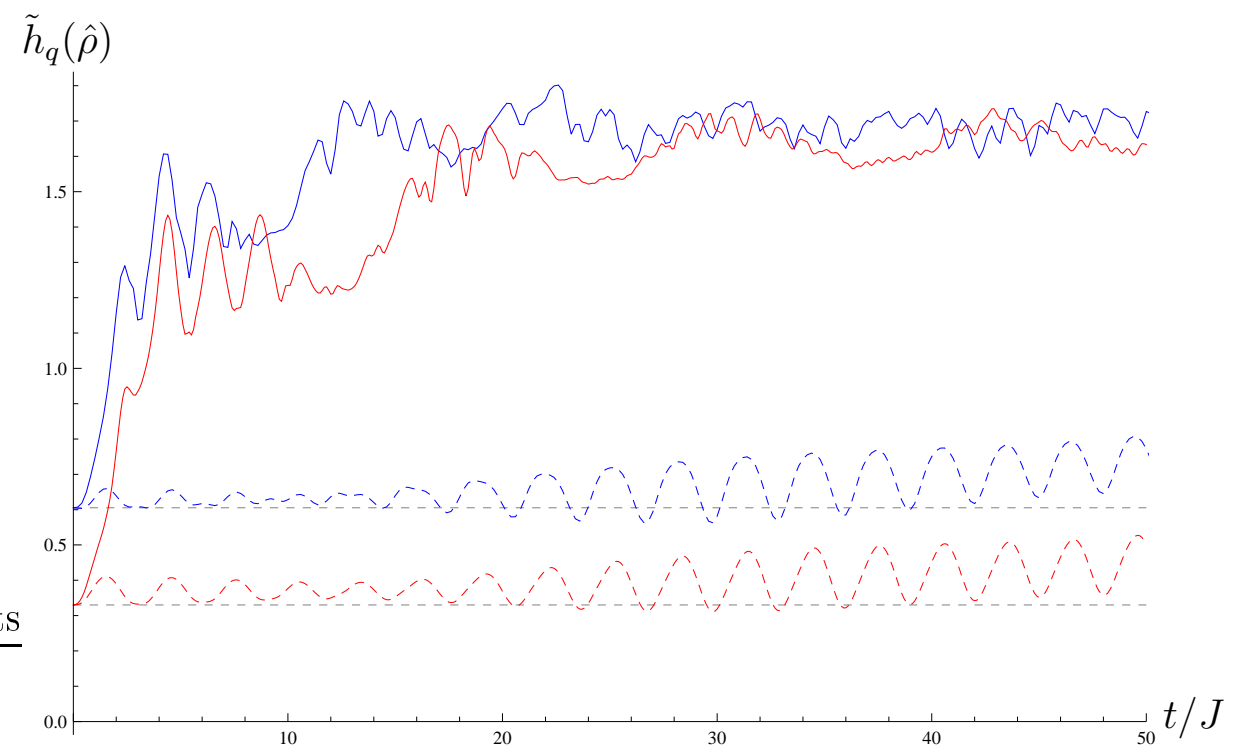

Figura 5.6: Heterogeneidade distribucional no espaço de fases como função do tempo escalonado $t / J$, para $N_{a}=N_{b}=10$ (azul) e 20 (vermelho), $\alpha=0.25$ e $M=3$. As diferentes texturas das curvas referem-se aos diferentes valores do parâmetro $\mu=0.1$ (tracejado) e 1.3 (contínuo). As linhas tracejadas horizontais correspondem aos valores iniciais de $\tilde{h}_{q}(\hat{\rho}) \approx 0.605274$ (azul) e 0.330368 (vermelho). 


\section{Capítulo 6}

\section{Conclusões e Perspectivas}

A heterogeneidade distribucional no espaço de fases, principal ferramenta utilizada em nosso estudo neste trabalho, foi proposta por Yan Gu [16] como uma medida da complexidade microscópica de distribuições no espaço de fases, tanto no caso de sistemas clássicos como no de sistemas quânticos. Uma outra característica desta medida de complexidade é que ela poder empregada como uma medida da (des)localização da distribuição no espaço de fases como em [24], também nos dois casos (clássico e quântico). No entanto, em ambos os contextos (clássico e quântico) ela foi originalmente concebida para tratar sistemas descritos em um espaço de dimensão infinita.

Neste trabalho, nós obtivemos uma extensão desta heterogeneidade distribucional no espaço de fases para sistemas descritos em um espaço de dimensão finita. Para tanto, utilizamos um procedimento extremamente geral, proposto por Schwinger [33] e extensivamente empregado na literatura (e.g. [21]), que se baseia exclusivamente na existência de uma base ortonormal no espaço de Hilbert do sistema considerado. Com esse procedimento foi possível obter "análogos" das quantidade dinâmicas relevantes à descrição destes sistemas, só que num espaço de dimensão finita. Uma destas quantidades dinâmicas é justamente a heterogeneidade distribucional no espaço de fases.

No caso de sistemas que envolvam um grande número de partículas, como aqueles que estudamos nesta tese, a propensão coletiva destes sistemas simplifica de forma considerável a obtenção das quantidade dinâmicas relevantes ao sistema, pois nos permite reduzir a dinâmica de um sistema com um número muito grande partículas à dinâmica de um sistema com poucos graus de liberdade, descritos por variáveis de natureza coletiva. Além disso, do ponto de vista da correspondência quântico-clássica, a propensão coletiva desempenha um papel fundamental no estudo destas quantidades dinâmicas. Mais precisamente, a possibilidade de podermos estudar o limite clássico do sistema em questão simplesmente fazendo um escalonamento das variáveis coletivas com o número de partículas $N$ e em seguida fazendo $N \rightarrow \infty$, nos permite analisar as propriedades de correspondência entre o sistema quântico e seu correspondente clássico de uma forma muito mais direta; permitindo, inclusive, "inferir" sobre certas propriedades (e.g., integrabilidade das equações 
de movimento) do sistema clássico já na análise do sistema quântico, que aliás é uma das vantagens desta forma de limite clássico sobre, por exemplo, estudos que envolvam diretamente procedimentos do tipo " $\hbar \rightarrow 0$ ", além de evitar certas dificuldades (por exemplo, escolha de ordenamento de operadores) intrínsecas ao uso de certos métodos de quantização.

Com tudo isso, realizar um estudo comparativo entre os comportamentos quântico e clássico destes "sistemas coletivos", por exemplo, através da análise do comportamento de distribuições (funções de Wigner) no espaço de fases, torna-se bastante imediato. Dentre os principais pontos que podemos destacar neste contexto está o fato do comportamento da heterogeneidade distribucional quântica para o caso de um espaço de dimensão finita $\tilde{h}_{q}(\hat{\rho})$ ter se mostrado em bom acordo com os resultados conhecidos na literatura [16, 18, 23, 19, 24], tanto para o caso de sistemas classicamente regulares como classicamente caóticos. De acordo com os resultados numéricos apresentados, vemos que a definição que utilizamos para descrever uma média (coarse-graining) discreta no espaço de fases (3.39), para o caso de um espaço de Hilbert de dimensão finita é razoavelmente sensível à variação da estrutura da função de Wigner no espaço de fases. Em particular, a análise desta variação de estrutura coloca em evidência de forma quantitativa a perda de coerência quântica através do processo de média (coarse-graining). Um estudo do comportamento de $\tilde{h}_{q}(\hat{\rho})$ para sistemas diferentes dos estudados aqui, por exemplo para sistemas abertos classicamente caóticos, como o sistema estudado em [7, 8, 9], seria contudo uma contribuição bastante interessante.

Um outro ponto que merece destaque neste trabalho é o efeito que a média (coarse-graining) no espaço de fases tem sobre as correlações quânticas na função de Wigner dos estados quânticos. O fato dos autovetores da matriz densidade correspondente a $\hat{\rho}_{\alpha}$ permenecerem deslocalizados é realmente surpreedente, assim como o fato deste efeito não perdurar ao longo da evolução temporal unitária do estado (ver figuras 4.9 - 4.11). Neste contexto, a figura 4.9 nos mostra que é possível recuperar com razoável fidelidade o estado inicial do sistema $\hat{\rho}_{0}$ mesmo após efetuada a média (coarse-graining) no espaço de fases, apenas olhando para os autovetores da matriz de $\hat{\rho}_{0 \alpha}$ (no caso da figura 4.9 o "overlap" entre o estado representado no gráfico (b) e o estado inicial (4.20) é $\simeq 0.993124$, enquanto que nas figuras 4.10 e 4.11 o "overlap" mais apreciável entre o estado antes da média (coarse-graining), representado por $\hat{\rho}$, e os autovetores da matriz de $\hat{\rho}_{\alpha}$ é dado respectivamente por $\simeq 0.960109 \mathrm{e} \simeq 0.701738$ ). Como mencionamos anteriormente, este fato indica que é preciso ser cuidadoso ao afirmar que a "eliminação total" do padrão oscilatório (estruturas de interferências) na função de Wigner do estado quântico, significa necessariamente que este tenha adquirido características clássicas.

Nas figuras $4.12,4.14,5.4$ e 5.6 vemos que o aumento do valor de $N$ afeta o comportamento de $\tilde{h}_{q}(\hat{\rho})$ predominantemente de forma quantitativa e que qualitativamente este comportamento pouco depende de $N$. Podemos resumir o comportamento qualitativo de $\tilde{h}_{q}(\hat{\rho})$ da seguinte forma: (i) para sistemas classicamente regulares (integráveis), $\tilde{h}_{q}(\hat{\rho})$ invariavelmente apresenta um comportamento oscilatório cujos períodos de oscilação variam conforme $N$ varia (ver figuras 4.12 e 4.14); (ii) para sistemas classicamente irregulares (caóticos), vemos que o comportamento de $\tilde{h}_{q}(\hat{\rho})$ praticamente 
não se altera conforme $N$ varia, ou seja, independentemente do valor de $N$ sempre ocorre um rápido crescimento inicial seguido de uma saturação no valor de $\tilde{h}_{q}(\hat{\rho})$ (ver figura 5.6).

Estes comportamentos (i) e (ii) são bastante razoáveis do ponto de vista quantitativo pois, conforme o valor de $N$ aumenta, a única diferença apreciável no espaço de fases é o aumento número de pontos deste aos quais a distribuição tem acesso, ou seja, ocorre um aumento no suporte da distribuição, o que possibilita que esta adquira mais estrutura. Podemos ver também, no gráfico de baixo da figura 4.12, que as curvas correspondentes aos diferentes valores de $N$ inicialmente praticamente coincidem e que isso ocorre durante um período curto de tempo; após esse período de tempo inicial as curvas se "separam" e assim seguem durante o restante da evolução de $\tilde{h}_{q}(\hat{\rho})$.

Devido a grande importância dos sistemas (4.3) e (5.1) para o estudo de sistemas de bósons armadilhados, que por sua vez é potencialmente relevante em áreas como teoria da informação quântica e óptica atômica [60] e o fato de que no estudo destas áreas um grande número de partículas é uma condição frequentemente necessária; é no mínimo extremamente desejável ter um conhecimento razoável e portanto um controle maior sobre o processo de transição quânticoclássica nestes sistemas, já que para estes sistemas a transição esta ligada justamente ao aumento do número de partículas. Esperamos com este trabalho ter contribuído significativamente neste sentido.

No contexto do estudo da correspondência quântico-clássica, a abordagem utilizada neste trabalho também poderia ser aplicada ao estudo de outros problemas relacionados ao comportamento de sistemas quânticos no limite clássico. Por exemplo, a relação entre o emaranhamento quântico e o comportamento de certos sistemas no espaço de fases clássico, como estudado em [56], ou a observação de emaranhamento quântico próximo ao limite clássico, como em [61].

Em outros contextos, seria interessante entender melhor o processo que leva à "não-deslocalização" dos estados, observada na figuras 4.9 - 4.11, e até que ponto a evolução unitária do sistema inluencia neste comportamento. Outro estudo interessante de ser feito é o da relação entre o comportamento da heterogeneidade distribucional no espaço de fases e a distribuição dos autovalores de energia. Como vimos anteriormente (figuras 5.4 e 5.5), estes comportamentos estão de alguma forma relacionados e um melhor entendimento da forma como eles se relacionam poderia nos permitir prever (ou até mesmo compreender melhor) o comportamento de um a partir do comportamento do outro. 


\section{Apêndices}

\section{A. Exemplo Explícito da Equivalência entre $\tilde{h}_{q}(\hat{\rho})$ e $h_{q}(\hat{\rho})$ no Limite $D \rightarrow \infty$ e da Não Comutatividade dos Limites $D \rightarrow \infty$ e $\alpha \rightarrow 0$}

Neste apêndice estudaremos, de uma forma um pouco mais explícita, a equivalência entre a definição da heterogeneidade distribucional no espaço de fases nos casos contínuo e discreto. Em especial, mostraremos como é possível passar do caso discreto para o caso contínuo através do limite $D \rightarrow \infty$. Também mostraremos de forma explícita, para um determinado exemplo de média, que os limites $D \rightarrow \infty$ e $\alpha \rightarrow 0$ não comutam.

Consideremos um sistema com apenas um grau de liberdade. Para um estado descrito por um operador densidade $\hat{\rho}$, podemos definir uma média (coarse-graining) sobre $\hat{\rho}$ da forma (2.42). Adotando para a entropia do sistema a definição (2.43), podemos utilizar (2.44) para definir a heterogeneidade distribucional no espaço de fases $h_{q}(\hat{\rho})(2.45)$.

Um ponto importante para esta análise reside no fato de que tomar explicitamente o limite $\alpha \rightarrow 0$ em (2.45)(1) equivale a calcularmos $(\hbar=1)$

$$
h_{q}(\hat{\rho}) \approx-\frac{1}{\operatorname{Tr}\left[\hat{\rho}^{2}\right]}\left(\left.\frac{\partial}{\partial \alpha} \operatorname{Tr}\left[\hat{\rho}_{\alpha}^{2}\right]\right|_{\alpha=0}\right),
$$

com

$$
\operatorname{Tr}\left[\hat{\rho}_{\alpha}^{2}\right]=\operatorname{Tr}\left[\int d \gamma \int d \gamma^{\prime} \delta_{\alpha}(\gamma) \delta_{\alpha}\left(\gamma^{\prime}\right) \mathrm{e}^{\mathrm{i}(\gamma \hat{\gamma})} \hat{\rho} \mathrm{e}^{-\mathrm{i}(\gamma \hat{\gamma})} \mathrm{e}^{\mathrm{i}\left(\gamma^{\prime} \hat{\gamma}\right)} \hat{\rho} \mathrm{e}^{-\mathrm{i}\left(\gamma^{\prime} \hat{\gamma}\right)}\right] .
$$

Utilizando as relações

$$
\mathrm{e}^{ \pm \mathrm{i}(\gamma \hat{\gamma})}=\mathrm{e}^{ \pm \mathrm{i}(-v \hat{p}+u \hat{q})}=\mathrm{e}^{\mp \mathrm{i}(v \hat{p})} \mathrm{e}^{ \pm \mathrm{i}(u \hat{q})} \mathrm{e}^{\mp \mathrm{i}\left(\frac{u v}{2}\right)},
$$

e substituindo (A.3) em (A.2), obtemos

$\operatorname{Tr}\left[\hat{\rho}_{\alpha}^{2}\right]=\operatorname{Tr}\left[\int d u d v \int d u^{\prime} d v^{\prime} \delta_{\alpha}(u, v) \delta_{\alpha}\left(u^{\prime}, v^{\prime}\right) \mathrm{e}^{-\mathrm{i}(v \hat{p})} \mathrm{e}^{\mathrm{i}(u \hat{q})} \hat{\rho} \mathrm{e}^{\mathrm{i}(v \hat{p})} \mathrm{e}^{-\mathrm{i}(u \hat{q})} \mathrm{e}^{-\mathrm{i}\left(v^{\prime} \hat{p}\right)} \mathrm{e}^{\mathrm{i}\left(u^{\prime} \hat{q}\right)} \hat{\rho} \mathrm{e}^{\mathrm{i}\left(v^{\prime} \hat{p}\right)} \mathrm{e}^{-\mathrm{i}\left(u^{\prime} \hat{q}\right)}\right]$. 
Finalmente, substituindo (A.4) em (A.1), temos que

$$
\begin{aligned}
h_{q}(\hat{\rho}) \approx & -\frac{1}{\operatorname{Tr}\left[\hat{\rho}^{2}\right]} \operatorname{Tr}\left[\int d u d v \int d u^{\prime} d v^{\prime}\left(\left.\frac{\partial}{\partial \alpha}\left\{\delta_{\alpha}(u, v) \delta_{\alpha}\left(u^{\prime}, v^{\prime}\right)\right\}\right|_{\alpha=0}\right) \times\right. \\
& \left.\times \mathrm{e}^{-\mathrm{i}(v \hat{p})} \mathrm{e}^{\mathrm{i}(u \hat{q})} \hat{\rho} \mathrm{e}^{\mathrm{i}(v \hat{p})} \mathrm{e}^{-\mathrm{i}(u \hat{q})} \mathrm{e}^{-\mathrm{i}\left(v^{\prime} \hat{p}\right)} \mathrm{e}^{\mathrm{i}\left(u^{\prime} \hat{q}\right)} \hat{\rho} \mathrm{e}^{\mathrm{i}\left(v^{\prime} \hat{p}\right)} \mathrm{e}^{-\mathrm{i}\left(u^{\prime} \hat{q}\right)}\right],
\end{aligned}
$$

que é a forma explícita de $h_{q}(\hat{\rho})(2.45)$ para o caso de um sistema com um grau de liberdade descrito em termos de variáveis $\hat{q}$ e $\hat{p}$ com espectro contínuo num espaço de dimensão infinita.

Consideremos agora (3.39), que pode ser vista como uma versão de (2.42) para um espaço $D$-dimensional, com $D$ finito. Neste caso, temos que $(M=D)$

$$
\operatorname{Tr}\left[\hat{\rho}_{\alpha}^{2}\right]=\operatorname{Tr}\left[\frac{1}{D^{2}} \sum_{m, n} \sum_{m^{\prime}, n^{\prime}} \Delta_{\alpha}(m, n) \Delta_{\alpha}\left(m^{\prime}, n^{\prime}\right) \hat{U}^{m} \hat{V}^{n} \hat{\rho} \hat{V}^{\dagger n} \hat{U}^{\dagger m} \hat{U}^{m^{\prime}} \hat{V}^{n^{\prime}} \hat{\rho} \hat{V}^{\dagger n^{\prime}} \hat{U}^{\dagger m^{\prime}}\right]
$$

Seguindo então o procedimento descrito na seção 3.1, podemos construir os operadores (3.8). Considerando $\delta=1$ e introduzindo a mudança de variáveis $u=\epsilon m$ e $v=-\epsilon n$, tal que $\Delta u=\Delta v=\epsilon$ com $\epsilon$ dado em (3.7), obtemos que

$$
\begin{aligned}
\operatorname{Tr}\left[\hat{\rho}_{\alpha}^{2}\right]= & \frac{1}{\left(D \epsilon^{2}\right)^{2}} \operatorname{Tr}\left[\sum_{u, v} \sum_{u^{\prime}, v^{\prime}} \Delta u \Delta v \Delta u^{\prime} \Delta v^{\prime} \Delta_{\alpha}\left(\frac{u}{\epsilon}, \frac{v}{\epsilon}\right) \Delta_{\alpha}\left(\frac{u^{\prime}}{\epsilon}, \frac{v^{\prime}}{\epsilon}\right) \times\right. \\
& \left.\times \mathrm{e}^{\mathrm{i} u \hat{Q}} \mathrm{e}^{-\mathrm{i} v \hat{P}} \hat{\rho} \mathrm{e}^{\mathrm{i} v \hat{P}} \mathrm{e}^{-\mathrm{i} u \hat{Q}} \mathrm{e}^{\mathrm{i} u^{\prime} \hat{Q}} \mathrm{e}^{-\mathrm{i} v^{\prime} \hat{P}} \hat{\rho} \mathrm{e}^{\mathrm{i} v^{\prime} \hat{P}} \mathrm{e}^{-\mathrm{i} u^{\prime} \hat{Q}}\right],
\end{aligned}
$$

com $u, v, u^{\prime}, v^{\prime} \in[-\epsilon(D-1) / 2, \epsilon(D-1) / 2]$. Além disso, utilizando o fato de que

$$
\mathrm{e}^{\mathrm{i} u \hat{Q}} \mathrm{e}^{-\mathrm{i} v \hat{P}}=\mathrm{e}^{-\mathrm{i} v \hat{P}} \mathrm{e}^{\mathrm{i} u \hat{Q}}
$$

temos que (A.7) fica

$$
\begin{aligned}
\operatorname{Tr}\left[\hat{\rho}_{\alpha}^{2}\right]= & \frac{1}{\left(D \epsilon^{2}\right)^{2}} \operatorname{Tr}\left[\sum_{u, v} \sum_{u^{\prime}, v^{\prime}} \Delta u \Delta v \Delta u^{\prime} \Delta v^{\prime} \Delta_{\alpha}\left(\frac{u}{\epsilon}, \frac{v}{\epsilon}\right) \Delta_{\alpha}\left(\frac{u^{\prime}}{\epsilon}, \frac{v^{\prime}}{\epsilon}\right) \times\right. \\
& \left.\times \mathrm{e}^{-\mathrm{i} v \hat{P}} \mathrm{e}^{\mathrm{i} u \hat{Q}} \hat{\rho} \mathrm{e}^{\mathrm{i} v \hat{P}} \mathrm{e}^{-\mathrm{i} u \hat{Q}} \mathrm{e}^{-\mathrm{i} v^{\prime} \hat{P}} \mathrm{e}^{\mathrm{i} u^{\prime} \hat{Q}} \hat{\rho} \mathrm{e}^{\mathrm{i} v^{\prime} \hat{P}} \mathrm{e}^{-\mathrm{i} u^{\prime} \hat{Q}}\right] .
\end{aligned}
$$

Levando em conta o fato de que no limite $D \rightarrow \infty$ temos que $\epsilon \rightarrow 0, \Delta u \rightarrow d u$ e $D \epsilon^{2} \rightarrow 2 \pi$ 
obtemos

$\operatorname{Tr}\left[\hat{\rho}_{\alpha}^{2}\right]=\frac{1}{(2 \pi)^{2}} \operatorname{Tr}\left[\int d u d v \int d u^{\prime} d v^{\prime} \tilde{\Delta}_{\alpha}(u, v) \tilde{\Delta}_{\alpha}\left(u^{\prime}, v^{\prime}\right) \mathrm{e}^{-\mathrm{i} v \hat{p}} \mathrm{e}^{\mathrm{i} u \hat{q}} \hat{\rho} \mathrm{e}^{\mathrm{i} v \hat{p}} \mathrm{e}^{-\mathrm{i} u \hat{q}} \mathrm{e}^{-\mathrm{i} v^{\prime} \hat{p}} \mathrm{e}^{\mathrm{i} u^{\prime} \hat{q}} \hat{\rho} \mathrm{e}^{\mathrm{i} v^{\prime} \hat{p}} \mathrm{e}^{-\mathrm{i} u^{\prime} \hat{q}}\right]$

onde $\Delta_{\alpha}\left(\frac{u}{\epsilon}, \frac{v}{\epsilon}\right) \underset{D \rightarrow \infty}{\longrightarrow} \tilde{\Delta}_{\alpha}(u, v)$. Levando em conta que quando $D \rightarrow \infty$ temos $v(\alpha) \propto \alpha$ (ver Apêndice B) e tomando o limite $\alpha \rightarrow 0$, obtemos

$$
\begin{aligned}
\tilde{h}_{q}(\hat{\rho}) \approx & -\frac{1}{(2 \pi)^{2} \operatorname{Tr}\left[\hat{\rho}^{2}\right]} \operatorname{Tr}\left[\int d u d v \int d u^{\prime} d v^{\prime}\left(\left.\frac{\partial}{\partial \alpha}\left\{\tilde{\Delta}_{\alpha}(u, v) \tilde{\Delta}_{\alpha}\left(u^{\prime}, v^{\prime}\right)\right\}\right|_{\alpha=0}\right) \times\right. \\
& \left.\times \mathrm{e}^{-\mathrm{i}(v \hat{p})} \mathrm{e}^{\mathrm{i}(u \hat{q})} \hat{\rho} \mathrm{e}^{\mathrm{i}(v \hat{p})} \mathrm{e}^{-\mathrm{i}(u \hat{q})} \mathrm{e}^{-\mathrm{i}\left(v^{\prime} \hat{p}\right)} \mathrm{e}^{\mathrm{i}\left(u^{\prime} \hat{q}\right)} \hat{\rho} \mathrm{e}^{\mathrm{i}\left(v^{\prime} \hat{p}\right)} \mathrm{e}^{-\mathrm{i}\left(u^{\prime} \hat{q}\right)}\right],
\end{aligned}
$$

que a menos de constantes é idêntica à (A.5), desde que $\tilde{\Delta}_{\alpha}(u, v)$ satisfaça às propriedades $(2.34)$ - (2.38).

Afim de verificar a não comutatividade dos limites $D \rightarrow \infty$ e $\alpha \rightarrow 0$, suponhamos para a função $\Delta_{\alpha}\left(\frac{u}{\epsilon}, \frac{v}{\epsilon}\right) \equiv \Delta_{\alpha}(\bar{u}, \bar{v})$ a forma

$$
\Delta_{\alpha}(\bar{u}, \bar{v})=C_{\alpha} \exp \left[-\frac{\left(\bar{u}^{2}+\bar{v}^{2}\right)}{\alpha}\right]
$$

onde

$$
C_{\alpha}=\left[\sum_{\bar{u}, \bar{v}} \Delta_{\alpha}(\bar{u}, \bar{v})\right]^{-1}
$$

Da propriedade (3.40) vem que

$$
\left.\frac{\partial}{\partial \alpha}\left\{\Delta_{\alpha}(\bar{u}, \bar{v}) \Delta_{\alpha}\left(\bar{u}^{\prime}, \bar{v}^{\prime}\right)\right\}\right|_{\alpha=0}=\left\{\left.\frac{\partial}{\partial \alpha} \Delta_{\alpha}(\bar{u}, \bar{v})\right|_{\alpha=0}\right\} D \delta_{\bar{u}^{\prime}, 0} \delta_{\bar{v}^{\prime}, 0}+D \delta_{\bar{u}, 0} \delta_{\bar{v}, 0}\left\{\left.\frac{\partial}{\partial \alpha} \Delta_{\alpha}\left(\bar{u}^{\prime}, \bar{v}^{\prime}\right)\right|_{\alpha=0}\right\}
$$

com

$$
\left.\frac{\partial}{\partial \alpha} \Delta_{\alpha}(\bar{u}, \bar{v})\right|_{\alpha=0}=\left.\left\{\left(\frac{\partial C_{\alpha}}{\partial \alpha}\right) \exp \left[-\left(\bar{u}^{2}+\bar{v}^{2}\right) / \alpha\right]+C_{\alpha}\left(\bar{u}^{2}+\bar{v}^{2}\right) \frac{\exp \left[-\left(\bar{u}^{2}+\bar{v}^{2}\right) / \alpha\right]}{\alpha^{2}}\right\}\right|_{\alpha=0},
$$

onde $(\bar{u}, \bar{v}) \in[-(D-1) / 2,(D-1) / 2]$. Para $D$ finito, como é o caso, vemos claramente que no limite $\alpha \rightarrow 0$

$$
C_{\alpha} \rightarrow 1
$$




$$
\begin{gathered}
\frac{\partial C_{\alpha}}{\partial \alpha} \rightarrow 0, \\
\exp \left[-\left(\bar{u}^{2}+\bar{v}^{2}\right) / \alpha\right] \rightarrow 0,
\end{gathered}
$$

e

$$
\frac{1}{\alpha^{2}} \exp \left[-\left(\bar{u}^{2}+\bar{v}^{2}\right) / \alpha\right] \rightarrow 0 \text {. }
$$

Consequentemente temos que o lado direito da expressão (A.12) se anula quando $\alpha \rightarrow 0$, indicando portanto que neste caso $\tilde{h}_{q}(\hat{\rho})$ seria identicamente nula. O que obviamente não ocorre quando tomamos primeiramente o limite $D \rightarrow \infty$ como podemos ver em (A.6) - (A.10). 


\section{B. Análise da variancia $v(\alpha)$ no Caso Contínuo e Obtenção da Equação (2.41)(2)}

Neste apêndice, estudaremos o comportamento da variancia $v(\alpha)$ para o caso de um sistema descrito por meio de variáveis $q$ - $p$ com espectro contínuo num espaço de dimensão infinita. Em seguida indicaremos como obter explicitamente a equação $(2.41)(2)$.

Consideremos então que a função de alisamento $\delta_{\alpha}(\gamma)$ em $(2.33)$ possui a seguinte forma

$$
\delta_{\alpha}(\gamma)=\frac{1}{\sqrt{\pi \alpha}} \exp \left[-\frac{\gamma^{2}}{\alpha}\right] .
$$

Em analogia com (3.48) e (3.49), podemos definir a variancia $v(\alpha)$ de $\delta_{\alpha}(\gamma)$ como

$$
v(\alpha) \equiv-\frac{1}{f(\alpha)}\left[\frac{d f(\alpha)}{d\left(\frac{1}{\alpha}\right)}\right]
$$

com

$$
f(\alpha)=\int \exp \left[-\frac{\gamma^{2}}{\alpha}\right] d \gamma=\sqrt{\pi \alpha}
$$

Neste caso temos

$$
\begin{gathered}
v(\alpha) \stackrel{(1)}{=}-\frac{1}{\sqrt{\pi \alpha}}\left[\frac{d}{d\left(\frac{1}{\alpha}\right)} \sqrt{\pi \alpha}\right] \\
\stackrel{(2)}{=} \frac{1}{2} \alpha
\end{gathered}
$$

ou seja, no caso contínuo $v(\alpha) \propto \alpha$. O fator $1 / 2 \mathrm{em}(\mathrm{B} .3)(2)$ não aparece quando consideramos, no caso de um sistema com apenas um grau de liberdade, as componentes de $\gamma \equiv(q, p)$, o que faz com que neste caso $v(\alpha)=\alpha$. Vale a pena enfatizar que este resultado vale tanto para o contexto clássico quanto para o contexto quântico.

Consideremos agora a definição de $\rho_{\alpha}(\gamma)$ dada em (2.33) com $\delta_{\alpha}\left(\gamma^{\prime}\right)$ da forma (B.1). Utilizando a aproximação $\gamma^{\prime} \sim \alpha \ll 1$ podemos escrever a seguinte expansão

$$
\rho\left(\gamma-\gamma^{\prime}\right)=\sum_{n=0}^{\infty}(-1)^{n} \frac{\left(\gamma^{\prime}\right)^{n}}{n !} \frac{\partial^{n} \rho(\gamma)}{\partial \gamma^{n}},
$$

que ao ser substituída em (2.33) nos dá

$$
\rho_{\alpha}(\gamma)=\sum_{n=0}^{\infty} \frac{(-1)^{n}}{n !} \frac{\partial^{n} \rho(\gamma)}{\partial \gamma^{n}} I_{n}(\alpha)
$$

onde 


$$
I_{n}(\alpha)=\int\left(\gamma^{\prime}\right)^{n} \delta_{\alpha}\left(\gamma^{\prime}\right) d \gamma^{\prime}
$$

Utilizando agora (B.4), (B.5), (B.1), o fato de que

$$
I_{0}(\alpha)=1, \quad I_{1}(\alpha)=0, \quad I_{n}(\alpha)=o(\alpha) \quad(n>2),
$$

e além disso o fato de que $I_{2}(\alpha)$ vem de $(2.37)$, podemos escrever $h_{c}(\rho)$ como

$$
h_{c}(\rho)=-\lim _{\alpha \rightarrow 0} \frac{1}{\int\left|\rho_{\alpha}(\gamma)\right|^{2} d \gamma}\left\{\int \rho(\gamma) \frac{\partial^{2} \rho(\gamma)}{\partial \gamma^{2}} d \gamma+O\left(\alpha^{2}\right)\right\},
$$

que após efetuarmos o limite $\alpha \rightarrow 0$ fica

$$
h_{c}(\rho) \approx-\frac{\int \rho(\gamma) \frac{\partial^{2} \rho(\gamma)}{\partial \gamma^{2}} d \gamma}{\int|\rho(\gamma)|^{2} d \gamma},
$$

que após uma integração por partes fica

$$
h_{c}(\rho) \approx \frac{\int\left|\frac{\partial \rho(\gamma)}{\partial \gamma}\right|^{2} d \gamma}{\int|\rho(\gamma)|^{2} d \gamma} .
$$

Que é exatamente a equação $(2.41)(2)$. 


\section{Obtenção de (2.45)(3) a partir de (3.39)}

Neste apêndice obteremos de forma explícita a equação (2.45)(3) tomando como ponto de partida a equação (3.39). No caso de um espaço de dimensão finita temos que a média (coarse-grainimg) é feita da seguinte forma

$$
\hat{\rho}_{\alpha}=\sum_{m, n} K_{\alpha}(m, n) \hat{U}^{m} \hat{V}^{n} \hat{\rho} \hat{V}^{-n} \hat{U}^{-m}
$$

onde os operadores $\hat{U}$ e $\hat{V}$ são definidos através de

$$
\begin{aligned}
& \hat{U}\left|v_{k}\right\rangle=\left|v_{k+1}\right\rangle, \quad \hat{U}\left|u_{k}\right\rangle=u_{k}\left|u_{k}\right\rangle \\
& \hat{V}\left|v_{k}\right\rangle=v_{k}\left|v_{k}\right\rangle, \quad \hat{V}\left|u_{k}\right\rangle=\left|u_{k-1}\right\rangle
\end{aligned}
$$

com $u_{k}=v_{k}=\exp (2 \pi \mathrm{i} k / D)$ e $D$ é a dimensão do espaço de Hilbert do sistema.

Sejam então os operadores $\hat{D}_{ \pm}$definidos da seguinte forma

$$
\hat{D}_{+}=\hat{U}-\hat{1} \quad \text { e } \quad \hat{D}_{-}=\hat{1}-\hat{V}
$$

onde $\hat{1}$ é o operador identidade. Utilizando (C.2), temos que (C.1) fica

$$
\hat{\rho}_{\alpha}=\sum_{m, n} \Delta_{\alpha}(m, n)\left(\hat{1}+\hat{D}_{+}\right)^{m}\left(\hat{1}-\hat{D}_{-}\right)^{n} \hat{\rho}\left(\hat{1}-\hat{D}_{-}\right)^{-n}\left(\hat{1}-\hat{D}_{+}\right)^{-m} .
$$

Consideremos agora as expansões

$$
\begin{gathered}
(x+a)^{\nu}=\sum_{k=0}^{\infty}\left(\begin{array}{l}
\nu \\
k
\end{array}\right) x^{k} a^{\nu-k}, \quad \nu \in \mathbb{R} \\
(x+a)^{-\nu}=\sum_{k=0}^{\infty}(-1)^{k}\left(\begin{array}{c}
\nu+k+1 \\
k
\end{array}\right) x^{k} a^{-\nu-k}, \quad \nu \in \mathbb{R} .
\end{gathered}
$$

Para caso $a=1$ estas expansões ficam

$$
(1+x)^{ \pm \nu}=\sum_{k=0}^{\infty} \frac{(\mp \nu)_{k}}{k !}(-x)^{k}
$$

onde $(\nu)_{k}$ é o símbolo de Pochhammer, cuja definição é

$$
(x)_{n} \equiv \frac{\Gamma(x+n)}{\Gamma(x)}
$$

onde $\Gamma(x)$ denota a função gama. 
Utilizando então a expansão (C.4) podemos escrever

$$
\begin{aligned}
& \left(\hat{1}+\hat{D}_{+}\right)^{ \pm m}=\sum_{k=0}^{\infty}( \pm 1) \frac{m^{k}}{k !} \hat{\Delta}_{+}^{k} \\
& \left(\hat{1}-\hat{D}_{-}\right)^{ \pm n}=\sum_{k=0}^{\infty}( \pm 1) \frac{n^{k}}{k !} \hat{\Delta}_{-}^{k}
\end{aligned}
$$

onde as potências dos operadores $\hat{\Delta}_{ \pm}$são dadas por

$$
\hat{\Delta}_{ \pm}^{k}=S_{k}^{(k)} \hat{D}_{ \pm}^{k}+\sum_{i=1}^{\infty}( \pm 1)^{i} \frac{S_{k+i}^{(i)}}{\prod_{j=1}^{i}(k+j)} \hat{D}_{ \pm}^{k+i}
$$

onde $S_{n}^{(k)}$ são os números de Stirling de primeiro tipo, os quais são definidos da seguinte forma

$$
\sum_{k=0}^{n} S_{n}^{(k)} x^{k}=(x)_{n}=(-1)^{n} n !\left(\begin{array}{c}
n-x-1 \\
n
\end{array}\right) .
$$

Consideremos agora uma função $K_{\alpha}(m, n)$ do tipo

$$
K_{\alpha}(m, n)=C(D, \alpha) \exp \left[-\frac{\left(m^{2}+n^{2}\right)}{\alpha}\right]
$$

onde $C(D, \alpha)$ é uma constante de normalização. Recorrendo novamente à aproximação $m, n \sim$ $\alpha \ll 1$, ao iserirmos (C.5) em (C.3) podemos desprezar os termos com potências de $m$ e $n$ maiores que dois. Com isso, após uma manipulação algébrica temos que (C.3) fica

$$
\begin{aligned}
\hat{\rho}_{\alpha} \approx & \sum_{m, n} K_{\alpha}(m, n)\left\{\hat{\rho}+\frac{1}{2} m^{2}\left[\hat{\Delta}_{+},\left[\hat{\Delta}_{+}, \hat{\rho}\right]\right]+\frac{1}{2} n^{2}\left[\hat{\Delta}_{-},\left[\hat{\Delta}_{-}, \hat{\rho}\right]\right]+\right. \\
& \left.+\frac{1}{4} m^{2} n^{2}\left[\hat{\Delta}_{+},\left[\hat{\Delta}_{+},\left[\hat{\Delta}_{-},\left[\hat{\Delta}_{-}, \hat{\rho}\right]\right]\right]\right]\right\}
\end{aligned}
$$

onde os comutadores $\left[\hat{\Delta}_{ \pm}, \hat{\rho}\right]$, através de (C.6), tem a forma

$$
\left[\hat{\Delta}_{ \pm}, \hat{\rho}\right]=\sum_{n=1}^{\infty}(-1)^{n-1} \frac{S_{n}^{(1)}}{n !} \sum_{k=1}^{n} \hat{D}_{ \pm}^{k-1}\left[\hat{D}_{ \pm}, \hat{\rho}\right] \hat{D}_{ \pm}^{n-k}
$$

Utilizando então (C.8) e (C.9), temos que 


$$
\begin{aligned}
\operatorname{Tr}\left\{\hat{\rho}_{\alpha}^{2}\right\} \approx & \operatorname{Tr}\left\{\hat{\rho}^{2}\right\} \sum_{m, n} \sum_{r, s} K_{\alpha}(m, n) K_{\alpha}(r, s)+ \\
& +\operatorname{Tr}\left\{\left[\hat{D}_{+}, \hat{\rho}\right]^{2}\right\} \sum_{m, n} \sum_{r, s} K_{\alpha}(m, n) K_{\alpha}(r, s) m^{2}+ \\
& +\operatorname{Tr}\left\{\left[\hat{D}_{-}, \hat{\rho}\right]^{2}\right\} \sum_{m, n} \sum_{r, s} K_{\alpha}(m, n) K_{\alpha}(r, s) n^{2},
\end{aligned}
$$

que de através da condição de normalização $\sum_{m, n} K_{\alpha}(m, n)=1$ e da definição dos operadores $\hat{D}_{ \pm}$ (C.2) fica

$$
\begin{aligned}
\operatorname{Tr}\left\{\hat{\rho}_{\alpha}^{2}\right\} \approx & \operatorname{Tr}\left\{\hat{\rho}^{2}\right\}+ \\
& +\operatorname{Tr}\left\{[\hat{U}, \hat{\rho}]^{2}\right\} \sum_{m, n} K_{\alpha}(m, n) m^{2}+ \\
& +\operatorname{Tr}\left\{[\hat{V}, \hat{\rho}]^{2}\right\} \sum_{m, n} K_{\alpha}(m, n) n^{2}
\end{aligned}
$$

Finalmente, lembrando que no caso de um espaço de dimensão finita a entropia do sistema é dada por (2.43), levando em conta $(2.45)(1)$ e empregando o procedimento apresentado na seção 3.1 podemos tomar o limite $D \rightarrow \infty$ em (C.11) e obter

$$
h(\hat{\rho}) \approx-\frac{1}{\operatorname{Tr}\left\{\hat{\rho}^{2}\right\}}\left(\operatorname{Tr}\left\{[\hat{Q}, \hat{\rho}]^{2}\right\}+\operatorname{Tr}\left\{[\hat{P}, \hat{\rho}]^{2}\right\}\right),
$$

que é o mesmo resultado de (2.45)(3), com $\hat{P}$ e $\hat{Q}$ definidos em (3.13) - (3.16). 


\section{Referências Bibliográficas}

[1] A. J. Leggett, J. Phys.: Cond. Mat. 14, R415 (2002).

[2] A. N. Salgueiro, M. C. Nemes, M. D. Sampaio, and A. F. R. de Toledo Piza, Physica A 290, 4 (2001).

[3] A. F. R. de Toledo Piza, Braz. Jour. Phys. 34, 1102 (2004).

[4] D. Galetti and A. F. R. de Toledo Piza, Physica A 149, 267 (1988).

[5] M. Ruzzi and D. Galetti, J. Phys. A:Math. Gen 33, 1065 (2000).

[6] M. Ruzzi, J. Phys. A:Math. Gen 35, 1763 (2002).

[7] W. K. Hensinger et al., Nature 412, 52 (2001).

[8] W. K. Steck et al., Science 293, 274 (2001).

[9] V. Averbukh, S. Osovski, and N. Moiseyev, Phys. Rev. Lett. 89, 253201 (2002).

[10] A. R. Kolovsky and A. Buchleitner, Europhys. Lett. 68, 632 (2004).

[11] K. M. Fonseca Romero, M. C. Nemes, J. G. Peixoto de Faria, and A. F. R. de Toledo Piza, Phys. Lett. A 327, 129 (2004).

[12] L. E. Ballentine, Phys. Rev. A 63, 024101 (2001).

[13] J. Emerson and L. E. Ballentine, Phys. Rev. E 64, 026217 (2001).

[14] J. Emerson and L. E. Ballentine, Phys. Rev. A 63, 052103 (2001).

[15] W. H. Zurek, Nature 412, 712 (2001).

[16] Y. Gu, Phys. Lett. A 149, 95 (1990).

[17] M. H. Partovi, Phys. Rev. A 45, R555 (1992).

[18] A. K. Pattanayak and P. Brumer, Phys. Rev. E 56, 5174 (1997). 
[19] J. Gong and P. Brumer, Phys. Rev. A 68, 062103 (2003).

[20] T. F. Viscondi, K. Furuya, and M. C. Oliveira, Ann. Phys. 324, 1837 (2009).

[21] D. Galetti and A. F. R. de Toledo Piza, Physica A 214, 207 (1995).

[22] I. G. Mata and M. Saraceno, Phys. Rev E 69, 056211 (2004).

[23] Q. S. Li, W. Y. Su, and J. Zou, Chaos, Solitons and Fractals 14, 975 (1997).

[24] H. J. Korsch and W. Leyes, New Journal of Physics 4, 62.1 (2002).

[25] A. K. Pattanayak et al., Phys. Rev. A 72, 013406 (2005).

[26] A. Gammal and A. K. Pattanayak, Phys. Rev. E 75, 036221 (2007).

[27] J.-P. Eckmann and D. Ruelle, Rev. Mod. Phys. 57, 617 (1985).

[28] G. Benettin, L. Galgani, and J.-M. Strelcyn, Phys. Rev. A. 14, 2338 (1976).

[29] H.-D. Meyer, J. Chem. Phys. 84, 3147 (1986).

[30] J. H. Hannay and M. V. Berry, Physica D 1, 267 (1980).

[31] Y. Gu and J. Wang, Phys. Lett. A 229, 208 (1997).

[32] L. Junqing, L. Fang, X. YongZhong, Z. Wei, and W. D. Heiss, Phys. Rev. E 65, 047203 (2002).

[33] J. Schwinger, Proc. Nat. Acad. Sci., USA 46, 570 (1960).

[34] J. Schwinger, Proc. Nat. Acad. Sci., USA 46, 893 (1960).

[35] J. Schwinger, Proc. Nat. Acad. Sci., USA 46, 1401 (1960).

[36] J. Schwinger, Proc. Nat. Acad. Sci., USA 47, 1075 (1961).

[37] D. Galetti and A. F. R. de Toledo Piza, Physica A 186, 513 (1992).

[38] M. Ruzzi and D. Galetti, J. Phys. A:Math. Gen 35, 4633 (2002).

[39] D. Ellinas and E. G. Floratos, J. Phys. A:Math. Gen. 32, L63 (1999).

[40] S. Nonnenmacher, Nonlinearity 16, 1685 (2003).

[41] M. Greiner, O. Mandel, T. Esslinger, T. W. Hänsch, and I. Bloch, Nature 415, 39 (2002).

[42] R. Franzosi and V. Penna, Phys. Rev A 63, 043609 (1997). 
[43] G. J. Milburn, J. Corney, E. M. Wright, and D. F. Walls, Phys. Rev A 55, 4318 (1997).

[44] J. R. Anglin and A. Vardi, Phys. Rev. A 64, 013605 (2001).

[45] M. Holthaus and S. Stenholm, Eur. Phys. J. B 20, 451 (2001).

[46] K. W. Mahmud, H. Perry, and W. P. Reinhardt, Phys. Rev. A 71, 023615 (2005).

[47] B. Wu and J. Liu, Phys. Rev. Lett. 96, 020405 (2006).

[48] J. Schwinger, L. Biederman, and H. Van Dam, Quantum Theory of Angular Momentum (Academic Press, New York, 1965).

[49] A. N. Salgueiro et al., Eur. Phys. J. D 44, 537 (2007).

[50] A. F. R. de Toledo Piza, Braz. Jour. Phys. 35, 122 (2005).

[51] L. van Hemmen and W. von Waldenfels, Physica A 100, 85 (1980).

[52] A. Perelomov, Generalized Coherente States and Their Applications (Springer-Verlag, Berlin, 1986).

[53] W. Zurek, Rev. Mod. Phys. 75, 715 (2003).

[54] M. Schlosshauer, Rev. Mod. Phys. 76, 1267 (2004).

[55] M. Schlosshauer, Decoherence and The Quantum-to-Classical Transition (Springer-Verlag, Berlin, 2007).

[56] A. P. Hines, R. H. McKenzie, and G. J. Milburn, Phys. Rev. A 71, 042303 (2005).

[57] W. Li, W. Yang, X. Xie, J. Li, and X. Yang, J. Phys. B: At. Mol. Opt. Phys. 39, 3097 (2006).

[58] H. T. Ng, C. K. Law, and P. T. Leung, Phys. Rev. A 68, 013604 (2003).

[59] H. T. Ng and P. T. Leung, Phys. Rev. A 71, 013601 (2005).

[60] J. I. Cirac, M. Lewenstein, K. Molmer, and P. Zoller, Phys. Rev. A 57, 1028 (1998).

[61] M. J. Everitt et al., New Journal of Physics 7, 64 (2005). 